\title{
Characterisation and mitigation of beam-induced backgrounds observed in the ATLAS detector during the 2011 proton-proton run
}

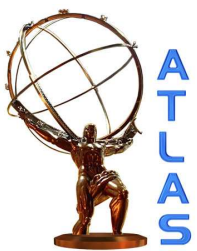

\section{The ATLAS collaboration}

E-mail: atlas.publications@cern.ch

ABSTRACT: This paper presents a summary of beam-induced backgrounds observed in the ATLAS detector and discusses methods to tag and remove background contaminated events in data. Triggerrate based monitoring of beam-related backgrounds is presented. The correlations of backgrounds with machine conditions, such as residual pressure in the beam-pipe, are discussed. Results from dedicated beam-background simulations are shown, and their qualitative agreement with data is evaluated. Data taken during the passage of unpaired, i.e. non-colliding, proton bunches is used to obtain background-enriched data samples. These are used to identify characteristic features of beam-induced backgrounds, which then are exploited to develop dedicated background tagging tools. These tools, based on observables in the Pixel detector, the muon spectrometer and the calorimeters, are described in detail and their efficiencies are evaluated. Finally an example of an application of these techniques to a monojet analysis is given, which demonstrates the importance of such event cleaning techniques for some new physics searches.

KEYWORDS: Pattern recognition, cluster finding, calibration and fitting methods; Performance of High Energy Physics Detectors; Accelerator modelling and simulations (multi-particle dynamics; single-particle dynamics); Analysis and statistical methods 


\section{Contents}

1 Introduction 1

2 LHC and the ATLAS interaction region $\quad 2$

3 The ATLAS detector $\quad 5$

4 Characteristics of BIB $\quad 8$

4.1 BIB simulation methods 9

5 BIB monitoring with Level-1 trigger rates 14

$\begin{array}{lll}5.1 & \text { BCM background rates vs residual pressure } & 15\end{array}$

$\begin{array}{ll}5.2 & \text { BCM background rates during } 2011\end{array}$

$\begin{array}{ll}5.3 \text { Observation of ghost charge } & 17\end{array}$

$\begin{array}{lll}5.4 & \text { Jet trigger rates in unpaired bunches } & 21\end{array}$

6 Studies of BIB with the ATLAS Pixel detector 22

6.1 Introduction 22

6.2 Pixel cluster properties 23

6.3 Pixel cluster compatibility method 25

6.4 BIB characteristics seen in 2011 data 29

7 BIB muon rejection tools $\quad 31$

7.1 General characteristics 31

7.2 BIB identification methods 35

$\begin{array}{lll}\text { 7.2.1 Segment method } & 35\end{array}$

$\begin{array}{lll}\text { 7.2.2 } & \text { One-sided method } & 35\end{array}$

$\begin{array}{lll}7.2 .3 & \text { Two-sided method } & 37\end{array}$

$\begin{array}{lll}\text { 7.2.4 Efficiency and mis-identification probability } & 37\end{array}$

$\begin{array}{lll}7.3 & \text { BIB rate in } 2011 & 38\end{array}$

8 Removal of non-collision background with jet observables $\quad 40$

8.1 Jet cleaning 40

8.1.1 Event samples $\quad 40$

8.1.2 Criteria to remove non-collision background 41

8.1.3 Evaluation of the jet quality selection efficiency 42

8.2 Monojet analysis 45

8.3 Summary of jet cleaning techniques 49

9 Conclusions $\quad 49$ 


\section{Introduction}

In this paper, analyses of beam induced backgrounds (BIB) seen in the ATLAS detector during the 2011 proton-proton run are presented. At every particle accelerator, including the LHC [1], particles are lost from the beam by various processes. During LHC high-luminosity running, the loss of beam intensity to proton-proton collisions at the experiments has a non-negligible impact on the beam lifetime. Beam cleaning, i.e. removing off-momentum and off-orbit particles is another important factor that reduces the beam intensity. Most of the cleaning losses are localised in special insertions far from the experiments, but a small fraction of the proton halo ends up on collimators close to the high-luminosity experiments. This distributed cleaning on one hand mitigates halo losses in the immediate vicinity of the experiments, but by intercepting some of the halo these collimators themselves constitute a source of background entering the detector areas.

Another important source of BIB is beam-gas scattering, which takes place all around the accelerator. Beam-gas events in the vicinity of the experiments inevitably lead to background in the detectors.

In ATLAS most of these backgrounds are mitigated by heavy shielding hermetically plugging the entrances of the LHC tunnel. However, in two areas of the detector, BIB can be a concern for operation and physics analyses:

- Background close to the beam-line can pass through the aperture left for the beam and cause large longitudinal clusters of energy deposition, especially in pixel detectors close to the interaction point (IP), increasing the detector occupancy and in extreme cases affecting the track reconstruction by introducing spurious clusters.

- High-energy muons are rather unaffected by the shielding material, but have the potential to leave large energy deposits via radiative energy losses in the calorimeters, where the energy gets reconstructed as a jet. These fake jets ${ }^{1}$ need to be identified and removed in physics analyses which rely on the measurement of missing transverse energy $\left(E_{\mathrm{T}}^{\mathrm{miss}}\right)$ and on jet identification. This paper presents techniques capable of tagging events with fake jets due to BIB.

An increase in occupancy due to BIB, especially when associated with large local charge deposition, can increase the dead-time of front-end electronics and lead to a degradation of datataking efficiency. In addition the triggers, especially those depending on $E_{\mathrm{T}}^{\text {miss }}$, can suffer from rate increases due to BIB.

\footnotetext{
${ }^{1}$ In this paper jet candidates originating from proton-proton collision events are called "collision jets" while jet candidates caused by BIB or other sources of non-collision backgrounds are referred to as "fake jets".
} 
This paper first presents an overview of the LHC beam structure, beam cleaning and interaction region layout, to the extent that is necessary to understand the background formation. A concise description of the ATLAS detector, with emphasis on the sub-detectors most relevant for background studies is given. This is followed by an in-depth discussion of BIB characteristics, presenting also some generic simulation results, which illustrate the main features expected in the data. The next sections present background monitoring with trigger rates, which reveal interesting correlations with beam structure and vacuum conditions. This is followed by background observations with the Pixel detector, which are compared with dedicated simulation results. The rest of the paper is devoted to fake-jet rates in the calorimeters and various jet cleaning techniques, which are effective with respect to BIB, but also other non-collisions backgrounds, like instrumental noise and cosmic muon induced showers.

\section{LHC and the ATLAS interaction region}

During the proton-proton run in 2011, the LHC operated at the nominal energy of $3.5 \mathrm{TeV}$ for both beams. The Radio-Frequency (RF) cavities, providing the acceleration at the LHC, operate at a frequency of $400 \mathrm{MHz}$. This corresponds to buckets every $2.5 \mathrm{~ns}$, of which nominally every tenth can contain a proton bunch. To reflect this sparse filling, groups of ten buckets, of which one can contain a proton bunch, are assigned the same Bunch Crossing IDentifier (BCID), of which there are 3564 in total. The nominal bunch spacing in the 2011 proton run was 50 ns, i.e. every second BCID was filled. Due to limitations of the injection chain the bunches are collected in trains, each containing up to 36 bunches. Typically four trains form one injected batch. The normal gap between trains within a batch is about $200 \mathrm{~ns}$, while the gap between batches is around $900 \mathrm{~ns}$. These train lengths and gaps are dictated by the injector chain and the injection process. In addition a $3 \mu \mathrm{s}$ long gap is left, corresponding to the rise-time of the kicker magnets of the beam abort system. The first BCID after the abort gap is by definition numbered as 1 .

A general layout of the LHC, indicating the interaction regions with the experiments as well as the beam cleaning insertions, is shown in figure 1.

The beams are injected from the Super Proton Synchrotron (SPS) with an energy of $450 \mathrm{GeV}$ in several batches and captured by the RF of the LHC. When the injection is complete the beams are accelerated to full energy. When the maximum energy is reached the next phase is the $\beta$-squeeze, ${ }^{2}$ during which the optics at the interaction points are changed from an injection value of $\beta^{*}=11 \mathrm{~m}$ to a lower value, i.e. smaller beam size, at the IP. Finally the beams are brought into collision, after which stable beams are declared and physics data-taking can commence. The phases prior to collisions, but at full energy, are relevant for background measurements because they allow the rates to be monitored in the absence of the overwhelming signal rate from the proton-proton interactions.

The number of injected bunches varied from about 200 in early 2011 to 1380 during the final phases of the 2011 proton-proton run. Typically, 95\% of the bunches were colliding in ATLAS. The pattern also included empty bunches and a small fraction of non-colliding, unpaired, bunches. Nominally the empty bunches correspond to no protons passing through ATLAS, and are useful for monitoring of detector noise. The unpaired bunches are important for background monitoring

\footnotetext{
${ }^{2}$ The $\beta$-function determines the variation of the beam envelope around the ring and depends on the focusing properties of the magnet lattice — for details see [3].
} 


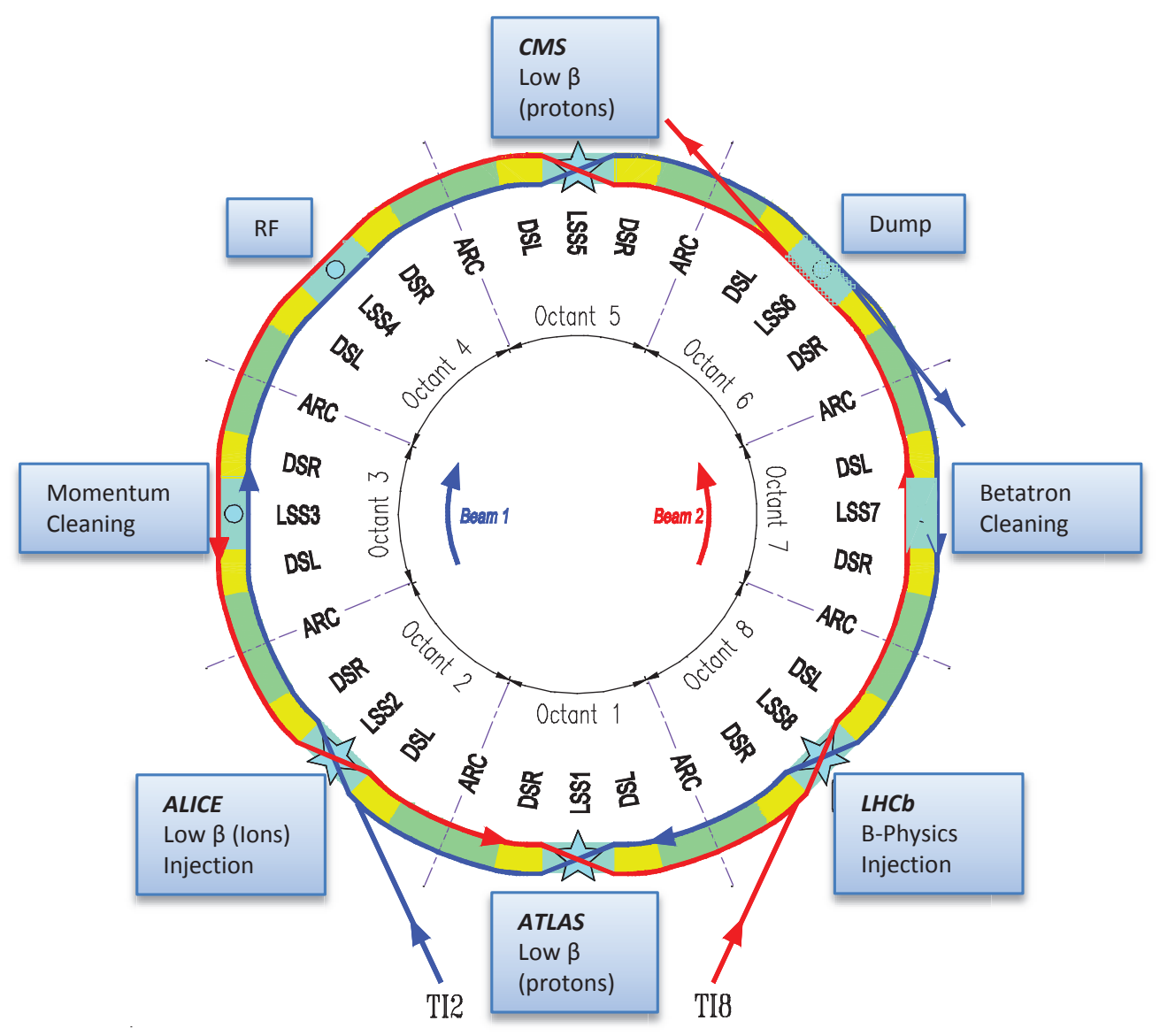

Figure 1. The general layout of the LHC [2]. The dispersion suppressors (DSL and DSR) are sections between the straight section and the regular arc. In this paper they are considered to be part of the arc, for simplicity. LSS denotes the Long Straight section - roughly $500 \mathrm{~m}$ long parts of the ring without net bending. All insertions (experiments, cleaning, dump, RF) are located in the middle of these sections. Beams are injected through transfer lines TI2 and TI8.

in ATLAS. It should be noted that these bunches were colliding in some other LHC experiments. They were introduced by shifting some of the trains with respect to each other, such that unpaired bunches appeared in front of a train in one beam and at the end in the other. In some fill patterns some of these shifts overlapped such that interleaved bunches with only $25 \mathrm{~ns}$ separation were introduced.

The average intensities of bunches in normal physics operation evolved over the year from $\sim 1.0 \times 10^{11} \mathrm{p} /$ bunch to $\sim 1.4 \times 10^{11} \mathrm{p} /$ bunch. The beam current at the end of the year was about $300 \mathrm{~mA}$ and the peak luminosity in ATLAS was $3.5 \times 10^{33} \mathrm{~cm}^{-2} \mathrm{~s}^{-1}$.

Due to the close bunch spacing, steering the beams head-on would create parasitic collisions outside of the IP. Therefore a small crossing angle is used; in 2011 the full angle was $240 \mu \mathrm{rad}$ in the vertical plane. In the high-luminosity interaction regions the number of collisions is maximised by the $\beta$-squeeze. In 2011 the value of $\beta^{*}$ was $1.5 \mathrm{~m}$ initially and was reduced to $1.0 \mathrm{~m}$ in midSeptember 2011. 


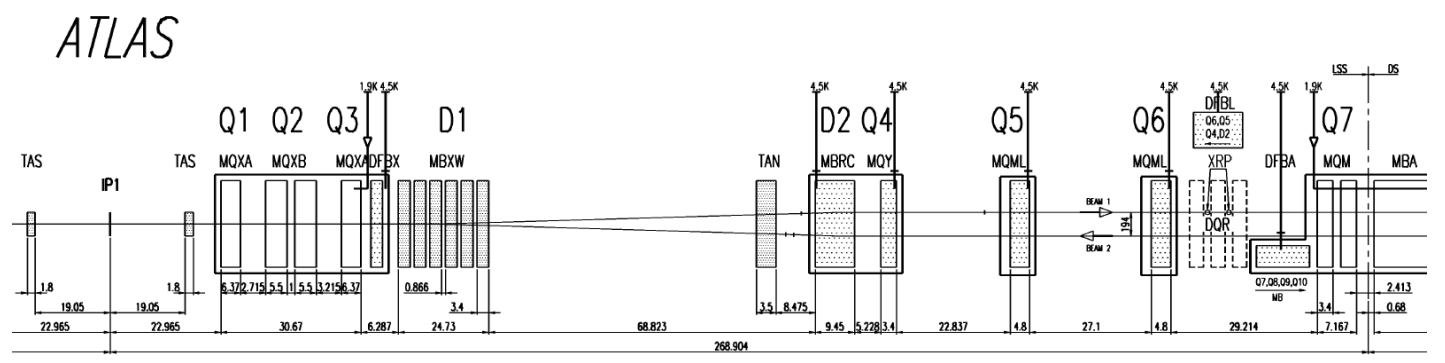

Figure 2. Detailed layout of the ATLAS interaction region [1]. The inner triplet consists of quadrupole magnets Q1, Q2 and Q3. The tertiary collimator (TCT) is not shown but is located between the neutral absorber (TAN) and the D2 magnet.

A detailed layout of the ATLAS interaction region (IR1) is shown in figure 2. Inside the inner triplet and up to the neutral absorber (TAN), both beams use the same beam pipe. In the arc, beams travel in separate pipes with a horizontal separation of $194 \mathrm{~mm}$. The separation and recombination of the beams happens in dipole magnets D1 and D2 with distances to the IP of 59-83 m and 153$162 \mathrm{~m}$, respectively. The D1 magnets are rather exceptional for the LHC, since they operate at room temperature in order to sustain the heat load due to debris from the interaction points. The TAS absorber, at $19 \mathrm{~m}$ from the IP, is a crucial element to protect the inner triplet against the heat load due to collision products from the proton-proton interactions. It is a $1.8 \mathrm{~m}$ long copper block with a $17 \mathrm{~mm}$ radius aperture for the beam. It is surrounded by massive steel shielding to reduce radiation levels in the experimental cavern [4]. The outer radius of this shielding extends far enough to cover the tunnel mouth entirely, thereby shielding ATLAS from low-energy components of BIB.

The large stored beam energy of the LHC, in combination with the heat sensitivity of the superconducting magnets, requires highly efficient beam cleaning. This is achieved by two separate cleaning insertions [6-8]: betatron cleaning at LHC point 7 and momentum cleaning at point 3 . In these insertions a two-stage collimation takes place, as illustrated in figure 3. Primary collimators (TCP) intercept particles that have left the beam core. Some of these particles are scattered and remain in the LHC acceptance, constituting the secondary halo, which hits the secondary collimators. Tungsten absorbers are used to intercept any leakage from the collimators. Although the combined local efficiency ${ }^{3}$ of the the system is better than $99.9 \%$ [8], some halo - called tertiary halo escapes and is lost elsewhere in the machine. The inner triplets of the high-luminosity experiments represent limiting apertures where losses of tertiary halo would be most likely. In order to protect the quadrupoles, dedicated tertiary collimators (TCT) were introduced at $145-148 \mathrm{~m}$ from the high-luminosity IP's on the incoming beam side. The tungsten jaws of the TCT were set in 2011 to $11.8 \sigma$, while the primary and secondary collimators at point 7 intercepted the halo at $5.7 \sigma$ and $8.5 \sigma$, respectively. ${ }^{4}$ Typical loss rates at the primary collimators were between $10^{8}-10^{9} \mathrm{p} / \mathrm{s}$ during the 2011 high luminosity operation. These rates are comparable to about $10^{8}$ proton-proton events/s in both ATLAS and CMS, which indicates that the beam lifetime was influenced about equally by halo losses and proton-proton collisions. The leakage fraction reaching the TCT was

\footnotetext{
${ }^{3}$ Here the local efficiency $\left(\varepsilon_{\text {loc }}\right)$ is defined such that on no element of the machine is the loss a fraction larger than $1-\varepsilon_{\text {loc }}$ of the total.

${ }^{4}$ Here $\sigma$ is the transverse betatronic beam standard deviation, assuming a normalised emittance of $3.5 \mu \mathrm{m}$. In 2011 the LHC operated at smaller than nominal emittance, thus the actual physical apertures were larger in terms of $\sigma$.
} 


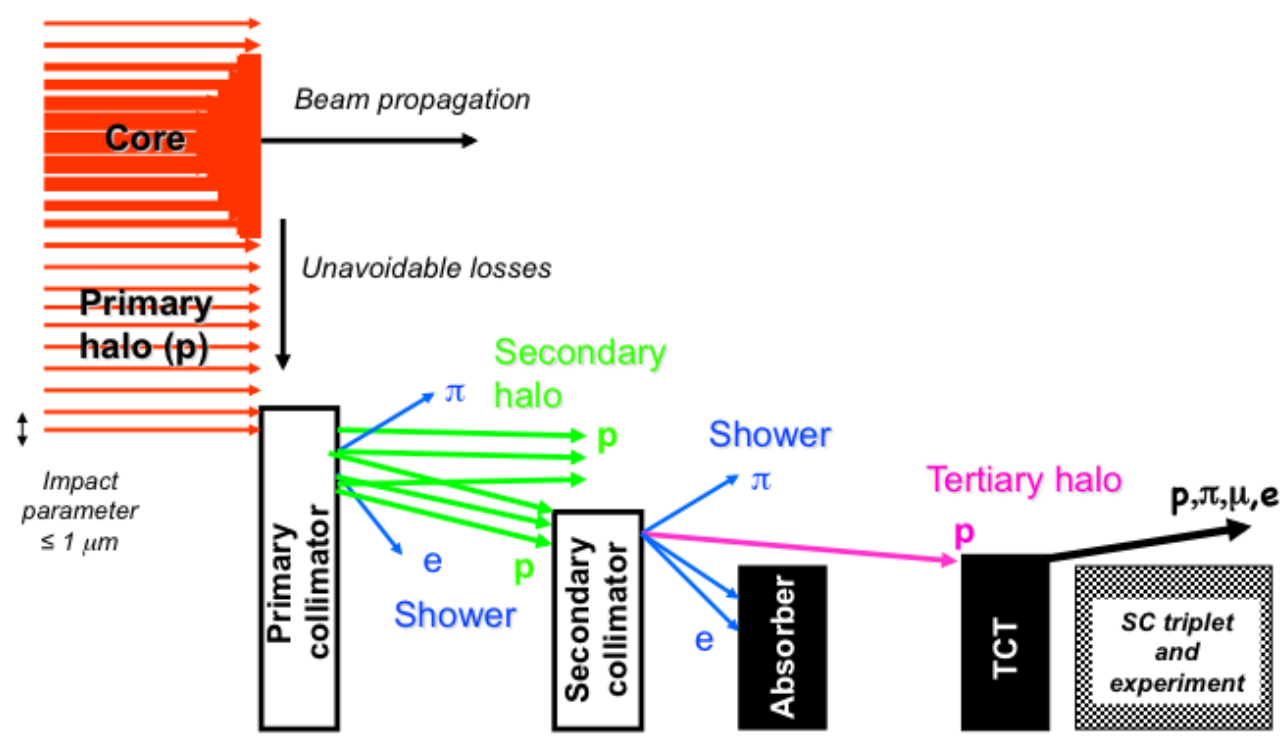

Figure 3. Schematic illustration of the LHC cleaning system. Primary and secondary collimators and absorbers in the cleaning insertions remove most of the halo. Some tertiary halo escapes and is intercepted close to the experiments by the TCT [5].

measured to be in the range $10^{-4}-10^{-3}[8,9]$, resulting in a loss rate on the order of $10^{5} \mathrm{p} / \mathrm{s}$ on the TCT.

The dynamic residual pressure, i.e. in the presence of a nominal beam, in the LHC beam pipe is typically of the order of $10^{-9}$ mbar $\mathrm{N}_{2}$-equivalent ${ }^{5}$ in the cold regions. In warm sections cryo-pumping, i.e. condensation on the cold pipe walls, is not available and pressures would be higher. Therefore most room-temperature sections of the vacuum chambers are coated with a special Non-Evaporative Getter (NEG) layer [10], which maintains a good vacuum and significantly reduces secondary electron yield. There are, however, some uncoated warm sections in the vicinity of the experiments. In 2010 and 2011 electron-cloud formation [11, 12] in these regions led to an increase of the residual pressure when the bunch spacing was decreased. As an emergency measure, in late 2010, small solenoids were placed around sections where electron-cloud formation was observed (58 $\mathrm{m}$ from the IP). These solenoids curled up the low-energy electrons within the vacuum, suppressing the multiplication and thereby preventing electron-cloud build-up. During a campaign of dedicated "scrubbing" runs with high-intensity injection-energy beams, the surfaces were conditioned and the vacuum improved. After this scrubbing, typical residual pressures in the warm sections remained below $10^{-8} \mathrm{mbar}_{2}$-equivalent in IR1 and were practically negligible in NEG coated sections — as predicted by early simulations [13].

\section{The ATLAS detector}

The ATLAS detector [14] at the LHC covers nearly the entire solid angle around the interaction point with calorimeters extending up to a pseudorapidity $|\eta|=4$.9. Here $\eta=-\ln (\tan (\theta / 2))$, with $\theta$ being the polar angle with respect to the nominal LHC beam-line.

\footnotetext{
${ }^{5}$ The most abundant gases are $\mathrm{H}_{2}, \mathrm{CO}, \mathrm{CO}_{2}$ and $\mathrm{CH}_{4}$. For simplicity a common practice is to describe these with an $\mathrm{N}_{2}$-equivalent, where the equivalence is calculated on the basis of the inelastic cross section at beam energy.
} 
In the right-handed ATLAS coordinate system, with its origin at the nominal IP, the azimuthal angle $\phi$ is measured with respect to the $x$-axis, which points towards the centre of the LHC ring. Side A of ATLAS is defined as the side of the incoming clockwise LHC beam-1, while the side of the incoming beam-2 is labelled C. The $z$-axis in the ATLAS coordinate system points from C to A, i.e. along the beam- 2 direction.

ATLAS consists of an inner tracking detector (ID) in the $|\eta|<2.5$ region inside a $2 \mathrm{~T}$ superconducting solenoid, which is surrounded by electromagnetic and hadronic calorimeters, and an external muon spectrometer with three large superconducting toroid magnets. Each of these magnets consists of eight coils arranged radially and symmetrically around the beam axis. The high- $\eta$ edge of the endcap toroids is at a radius of $0.83 \mathrm{~m}$ and they extend to a radius of $5.4 \mathrm{~m}$. The barrel toroid is at a radial distance beyond $4.3 \mathrm{~m}$ and is thus not relevant for studies in this paper.

The ID is responsible for the high-resolution measurement of vertex positions and momenta of charged particles. It comprises a Pixel detector, a silicon tracker (SCT) and a Transition Radiation Tracker (TRT). The Pixel detector consists of three barrel layers at mean radii of $50.5 \mathrm{~mm}$, $88.5 \mathrm{~mm}$ and $122.5 \mathrm{~mm}$ each with a half-length of $400.5 \mathrm{~mm}$. The coverage in the forward region is provided by three Pixel disks per side at $z$-distances of $495 \mathrm{~mm}, 580 \mathrm{~mm}$ and $650 \mathrm{~mm}$ from the IP and covering a radial range between $88.8-149.6 \mathrm{~mm}$. The Pixel sensors are $250 \mu \mathrm{m}$ thick and have a nominal pixel size of $r \phi \times z=50 \times 400 \mu \mathrm{m}^{2}$. At the edge of the front-end chip there are linked pairs of "ganged" pixels which share a read-out channel. These ganged pixels are typically excluded in the analyses presented in this paper.

The ATLAS solenoid is surrounded by a high-granularity liquid-argon (LAr) electromagnetic calorimeter with lead as absorber material. The LAr barrel covers the radial range between $1.5 \mathrm{~m}$ and $2 \mathrm{~m}$ and has a half-length of $3.2 \mathrm{~m}$. The hadronic calorimetry in the region $|\eta|<1.7$ is provided by a scintillator-tile calorimeter (TileCal), while hadronic endcap calorimeters (HEC) based on LAr technology are used in the region $1.5<|\eta|<3.2$. The absorber materials are iron and copper, respectively. The barrel TileCal extends from $r=2.3 \mathrm{~m}$ to $r=4.3 \mathrm{~m}$ and has a total length of $8.4 \mathrm{~m}$. The endcap calorimeters cover up to $|\eta|=3.2$, beyond which the coverage is extended by the Forward Calorimeter (FCAL) up to $|\eta|=4.9$. The high- $\eta$ edge of the FCAL is at a radius of $\sim 70 \mathrm{~mm}$ and the absorber materials are copper (electromagnetic part) and tungsten (hadronic part). Thus the FCAL is likely to provide some shielding from BIB for the ID. All calorimeters provide nanosecond timing resolution.

The muon spectrometer surrounds the calorimeters and is composed of a Monitored Drift Tube (MDT) system, covering the region of $|\eta|<2.7$ except for the innermost endcap layer where the coverage is limited to $|\eta|<2$. In the $|\eta|>2$ region of the innermost layer, Cathode-Strip Chambers (CSC) are used. The CSCs cover the radial range 1-2 $\mathrm{m}$ and are located at $|z|=8 \mathrm{~m}$ from the IP. The timing resolution of the muon system is $2.5 \mathrm{~ns}$ for the MDT and $7 \mathrm{~ns}$ for the CSC. The firstlevel muon trigger is provided by Resistive Plate Chambers (RPC) up to $|\eta|=1.05$ and Thin Gap Chambers (TGC) for $1.05<|\eta|<2.4$.

Another ATLAS sub-detector extensively used in beam-related studies is the Beam Conditions Monitor (BCM) [15]. Its primary purpose is to monitor beam conditions and detect anomalous beam-losses which could result in detector damage. Aside from this protective function it is also used to monitor luminosity and BIB levels. It consists of two detector stations (forward and backward) with four modules each. A module consists of two polycrystalline chemical-vapour- 
deposition (pCVD) diamond sensors, glued together back-to-back and read out in parallel. The modules are positioned at $z= \pm 184 \mathrm{~cm}$, corresponding to $z / c=6.13 \mathrm{~ns}$ distance to the interaction point. The modules are at a radius of $55 \mathrm{~mm}$, i.e. at an $|\eta|$ of about 4.2 and arranged as a cross - two modules on the vertical axis and two on the horizontal. The active area of each sensor is $8 \times 8 \mathrm{~mm}^{2}$. They provide a time resolution in the sub-ns range, and are thus well suited to identify BIB by timing measurements.

In addition to these main detectors, ATLAS has dedicated detectors for forward physics and luminosity measurement (ALFA, LUCID, ZDC), of which only LUCID was operated throughout the 2011 proton run. Despite the fact that LUCID is very close to the beam-line, it is not particularly useful for background studies, mainly because collision activity entirely masks the small background signals.

An ATLAS data-taking session (run) ideally covers an entire stable beam period, which can last several hours. During this time beam intensities and luminosity, and thereby the event rate, change significantly. To optimise the data-taking efficiency, the trigger rates are adjusted several times during a run by changing the trigger prescales. To cope with these changes and those in detector conditions, a run is subdivided into luminosity blocks (LB). The typical length of a LB in the 2011 proton-proton run was 60 seconds. The definition contains the intrinsic assumption that during a LB the luminosity changes by a negligible amount. Changes to trigger prescales and any other settings affecting the data-taking are always aligned with LB boundaries.

In order to assure good quality of the analysed data, lists of runs and LBs with good beam conditions and detector performance are used. Furthermore, there are quality criteria for various reconstructed physics objects in the events that help to distinguish between particle response and noise. In the context of this paper, it is important to mention the quality criteria related to jets reconstructed in the calorimeters. The jet candidates used here are reconstructed using the anti- $k_{t}$ jet clustering algorithm [16] with a radius parameter $R=0.4$, and topologically connected clusters of calorimeter cells [17] are used as input objects. Energy deposits arising from particles showering in the calorimeters produce a characteristic pulse in the read-out of the calorimeter cells that can be used to distinguish ionisation signals from noise. The measured pulse is compared with the expectation from simulation of the electronics response, and the quadratic difference $Q_{\text {cell }}$ between the actual and expected pulse shape is used to discriminate noise from real energy deposits. ${ }^{6}$ Several jet-level quantities can be derived from the following cell-level variables:

- $f_{\mathrm{HEC}}$. Fraction of the jet energy in the HEC calorimeter.

- $\langle Q\rangle$. The average jet quality is defined as the energy-squared weighted average of the pulse quality of the calorimeter cells $\left(Q_{\text {cell }}\right)$ in the jet. This quantity is normalised such that $0<$ $\langle Q\rangle<1$.

${ }^{6} Q_{\text {cell }}$ is computed online using the measured samples of the pulse shape in time as

$$
Q_{\text {cell }}=\sum_{j=1}^{N}\left(s_{j}-A g_{j}^{\text {phys }}\right)^{2}
$$

where $A$ is the measured amplitude of the signal [18], $s_{j}$ is the amplitude of each sample $j$, and $g_{j}^{\text {phys }}$ is the normalised predicted ionisation shape. 
- $f_{\mathrm{Q}}^{\mathrm{LAr}}$. Fraction of the energy in LAr calorimeter cells with poor signal shape quality $\left(Q_{\text {cell }}>\right.$ 4000).

- $f_{\mathrm{Q}}^{\mathrm{HEC}}$. Fraction of the energy in the HEC calorimeter cells with poor signal shape quality $\left(Q_{\text {cell }}>4000\right)$.

- $E_{\text {neg. }}$ Energy of the jet originating from cells with negative energy that can arise from electronic noise or early out-of-time pile-up. ${ }^{7}$

\section{Characteristics of BIB}

At the LHC, BIB in the experimental regions are due mainly to three different processes [19-21]:

- Tertiary halo: protons that escape the cleaning insertions and are lost on limiting apertures, typically the TCT situated at $|z| \approx 150 \mathrm{~m}$ from the IP.

- Elastic beam-gas: elastic beam-gas scattering, as well as single diffractive scattering, can result in small-angle deflections of the protons. These can be lost on the next limiting aperture before reaching the cleaning insertions. These add to the loss rate on the TCTs.

- Inelastic beam-gas: inelastic beam-gas scattering results in showers of secondary particles. Most of these have only fairly local effects, but high-energy muons produced in such events can travel large distances and reach the detectors even from the LHC arcs.

By design, the TCT is the main source of BIB resulting from tertiary halo losses. Since it is in the straight section with only the D1 dipole and inner triplet separating it from the IP, it is expected that the secondary particles produced in the TCT arrive at rather small radii at the experiment. The losses on the TCT depend on the leakage from the primary collimators, but also on other bottlenecks in the LHC ring. Since the betatron cleaning is at LHC point 7, halo of the clockwise beam-1 has to pass two LHC octants to reach ATLAS, while beam-2 halo has six octants to cover, with the other low- $\beta$ experiment, CMS, on the way. Due to this asymmetry, BIB due to losses on the TCT cannot be assumed to be symmetric for both beams.

There is no well-defined distinction between halo and elastic beam-gas scattering because scattering at very small angles feeds the halo, the formation of which is a multi-turn process as protons slowly drift out of the beam core until they hit the primary collimators in the cleaning insertions at IP3 and IP7. Some scattering events, however, lead to enough deflection that the protons are lost on other limiting apertures before they reach the cleaning insertions. The most likely elements at which those protons can be lost close to the experiments are the TCTs. The rate of such losses is in addition to the regular tertiary halo. This component is not yet included in the simulations, but earlier studies based on $7 \mathrm{TeV}$ beam energy suggest that it is of similar magnitude as the tertiary halo [20]. The same $7 \mathrm{TeV}$ simulations also indicate that the particle distributions at the experiment are very similar to those due to tertiary halo losses.

The inelastic beam-gas rate is a linear function of the beam intensity and of the residual pressure in the vacuum chamber. The composition of the residual gas depends on the surface characteristics of the vacuum chamber and is different in warm and cryogenic sections and in those with

\footnotetext{
${ }^{7}$ Out-of-time pile-up refers to proton-proton collisions occurring in BCIDs before or after the triggered collision event.
} 


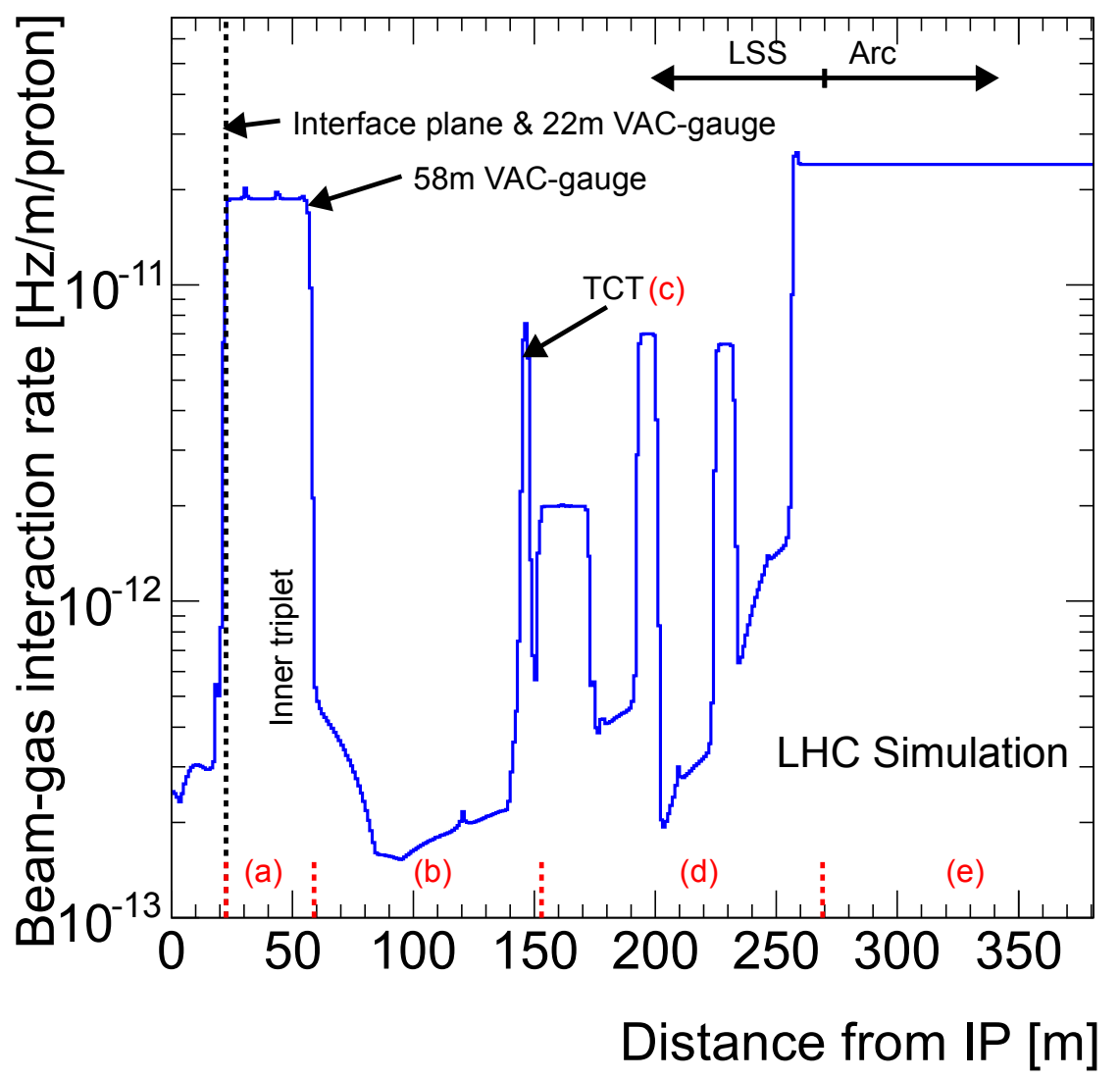

Figure 4. Inelastic beam-gas interaction rates per proton, calculated for beam-1 in LHC fill 2028. The machine elements of main interest are indicated. The pressure in the arc is assumed to be constant from $270 \mathrm{~m}$ onwards. The letters, a-e, identify the different sections, for which rates are given separately in other plots.

NEG coating. Although several pressure gauges are present around the LHC, detailed pressure maps can be obtained only from simulation similar to those described in [22]. The gauges can then be used to cross-check the simulation results at selected points. The maps allow the expected rate of beam-gas events to be determined. Such an interaction distribution, calculated for the conditions of LHC fill 2028, is shown in figure 4. The cryogenic regions, e.g. inner triplet (23-59m), the magnets D2 \& Q4, Q5 and Q6 at $\sim 170 \mathrm{~m}, \sim 200 \mathrm{~m}$ and $\sim 220 \mathrm{~m}$, respectively, and the arc $(>269 \mathrm{~m})$, are clearly visible as regions with a higher rate, while the NEG coating of warm sections efficiently suppresses beam-gas interactions. The TCT, being a warm element without NEG coating, produces a prominent spike at $\sim 150 \mathrm{~m}$. In the simulations it is assumed that the rate and distribution of beam-gas events are the same for both beams.

\subsection{BIB simulation methods}

The simulation of BIB follows the methods first outlined in [19], in particular the concept of a two-phase approach with the machine and experiment simulations being separate steps. In the first phase the various sources of BIB are simulated for the LHC geometry [9, 21]. These simulations produce a file of particles crossing an interface plane at $z=22.6 \mathrm{~m}$ from the IP. From this plane 
onwards, dedicated detector simulations are used to propagate the particles through the experimental area and the detector. Contrary to earlier studies [19, 20, 23], more powerful CPUs available today allow the machine simulations to be performed without biasing. ${ }^{8}$ This has the advantage of preserving all correlations within a single event and thus allows event-by-event studies of detector response. The beam halo formation and cleaning are simulated with SixTrack [24], which combines optical tracking and Monte Carlo simulation of particle interactions in the collimators. The inelastic interactions, either in the TCT based on the impact coordinates from SixTrack, or with residual gas, are simulated with FLUKA [25]. The further transport of secondary particles up to the interface plane is also done with FLUKA.

High-energy muons are the most likely particles to cause fake jet signals in the calorimeters. At sufficiently large muon energies, typically above $100 \mathrm{GeV}$, radiative energy losses start to dominate and these can result in local depositions of a significant fraction of the muon energy via electromagnetic and, rarely, hadronic cascades [26].

The TCTs are designed to intercept the tertiary halo. Thus they represent intense — viewed from the IP, almost point-like - sources of high-energy secondary particles. The TCTs are in the straight section and the high-energy particles have a strong Lorentz boost along $z$. Although they have to traverse the D1 magnet and the focusing quadrupoles before reaching the interface plane, most of the muons above $100 \mathrm{GeV}$ remain at radii below $2 \mathrm{~m}$.

The muons from inelastic beam-gas events, however, can originate either from the straight section or from the arc. In the latter case they emerge tangentially to the ring or pass through several bending dipoles, depending on energy and charge. Both effects cause these muons to be spread out in the horizontal plane so that their radial distribution at the experiment shows long tails, especially towards the outside of the ring.

In the following, some simulation results are shown, based on the distribution of muons with momentum greater than $100 \mathrm{GeV}$ at the interface plane. The reason to restrict the discussion to muons is twofold:

1. The region between the interface plane and the IP is covered by heavy shielding and detector material. All hadrons and EM-particles, except those within the $17 \mathrm{~mm}$ TAS aperture or at radii outside the shielding, undergo scattering and result in a widely spread shower of secondary particles. Therefore the distributions of these particles at the interface plane do not directly reflect what can be seen in the detector data.

2. High-energy muons are very penetrating and rather unaffected by material, but they are also the cause of beam-related calorimeter background. Therefore the distribution of high-energy muons is expected to reflect the fake jet distribution seen in data. The muon component is less significant for the ID, but its distribution can still reveal interesting effects.

Figure 5 shows the simulated $z$-distribution of inelastic beam-gas events resulting in a highenergy muon at the interface plane. In order to reach larger radii the muons have to originate from

\footnotetext{
${ }^{8}$ There are several biasing techniques available in Monte Carlo simulations. All of these aim at increasing statistics in some regions of phase space at the cost of others by modifying the physical probabilities and compensating this by assigning non-unity statistical weights to the particles. As an example the life-time of charged pions can be decreased in order to increase muon statistics. The statistical weight of each produced muon is then smaller than one so that on average the sum of muon weights corresponds to the true physical production rate.
} 


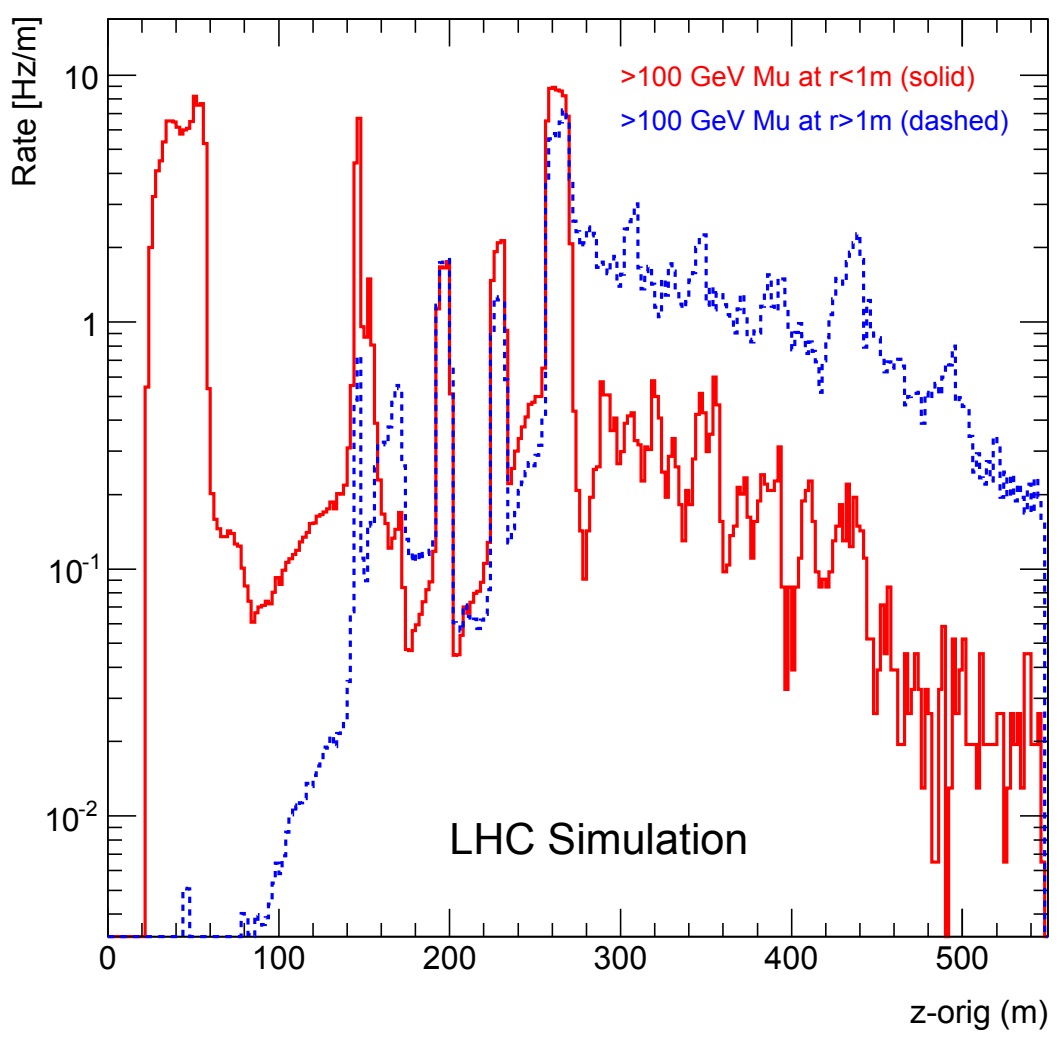

Figure 5. Simulated distribution of the $z$-coordinates of inelastic beam-gas events from which a muon with more than $100 \mathrm{GeV}$ has reached the interface plane at $22.6 \mathrm{~m}$. The two curves correspond to muons at radii below and above $1 \mathrm{~m}$ at the interface plane.

more distant events. Since the barrel calorimeters, ${ }^{9}$ which detect the possible fake jets, cover radii above $1 \mathrm{~m}$, the fake jet rate is not expected to be sensitive to close-by beam-gas interactions and therefore not to the pressure in the inner triplet. This is discussed later in the context of correlations between background rates and pressures seen by the vacuum gauges at $|z|=22 \mathrm{~m}$ and $|z|=58 \mathrm{~m}$.

Figure 6 shows the simulated radial distributions of high-energy muons from inelastic beamgas events taking place at various distances from the IP. Figure 4 suggests that the regions with highest interaction rate are the inner triplet, the TCT region, the cold sections in the LSS beyond the TCT, and the arc. In NEG-coated warm regions the expected beam-gas rate is negligible, which allows the interesting sections to be grouped into four wide regions, as indicated at the bottom of figure 4. It is evident from figure 6 that at very small radii beam-gas interactions in the inner triplet dominate, but these do not give any contributions at radii beyond $1 \mathrm{~m}$. The radial range between 1-4 m, covered by the calorimeters, gets contributions from all three distant regions, but the correlation between distance and radius is very strong and in the TileCal $(r=2-4 \mathrm{~m})$ muons from the arc dominate by a large factor. Beyond a radius of $4 \mathrm{~m}$ only the arc contributes to the high-energy muon rate.

\footnotetext{
${ }^{9}$ Fake jets can be produced also in the endcap and forward calorimeters, but due to higher rapidity are less likely to fake a high- $p_{\mathrm{T}}$ jet.
} 


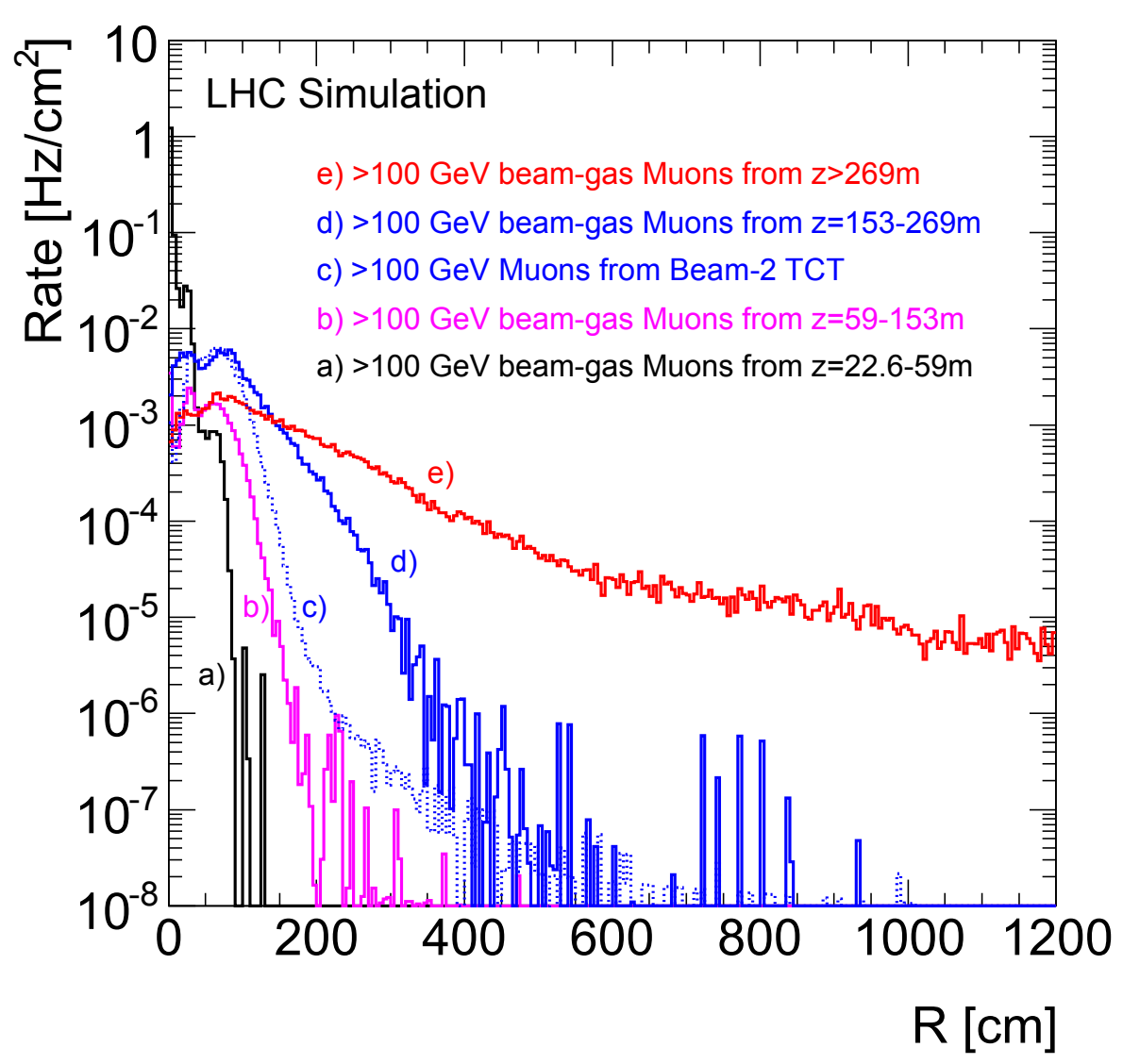

Figure 6. Simulated radial distribution at the interface plane of muons from inelastic beam-gas collisions and from the beam-2 TCT. The four solid curves correspond to muons originating from beam-gas events in different regions of the LSS and the adjacent LHC arc. The dashed curve shows the distribution of muons from the beam-2 TCT, normalised to $10^{5} \mathrm{p} / \mathrm{s}$ lost on the TCT. The letters refer to the regions indicated in figure 4 .

The dashed curve in figure 6 shows the radial distribution of high-energy muons from interactions in the TCT, which represents a practically point-like source situated at slightly less than $150 \mathrm{~m}$ from the IP. It can be seen that the radial distribution is quite consistent with that of beam-gas collisions in the $z=59-153 \mathrm{~m}$ region. The TCT losses lead to a fairly broad maximum below $r=1 \mathrm{~m}$, followed by a rapid drop, such that there are very few high-energy muons from the TCT at $r>3 \mathrm{~m}$. The absolute level, normalised to the average loss rate of $10^{5} \mathrm{p} / \mathrm{s}$ on the TCT, is comparable to that expected from beam-gas collisions.

Figure 7 shows the simulated $\phi$-distribution of the high-energy muons for different radial ranges and regions of origin of the muons. At radii below $1 \mathrm{~m}$ the muons from the inner triplet show a structure with four spikes, created by the quadrupole fields of the focusing magnets. Muons from more distant locations are deflected in the horizontal plane by the separation and recombination dipoles creating a structure with two prominent spikes. The figure shows both charges together, but actually D1 separates, according to charge, the muons originating from within 59-153 m. Since D2 has the same bending power but in the opposite direction, muons from farther away are again 

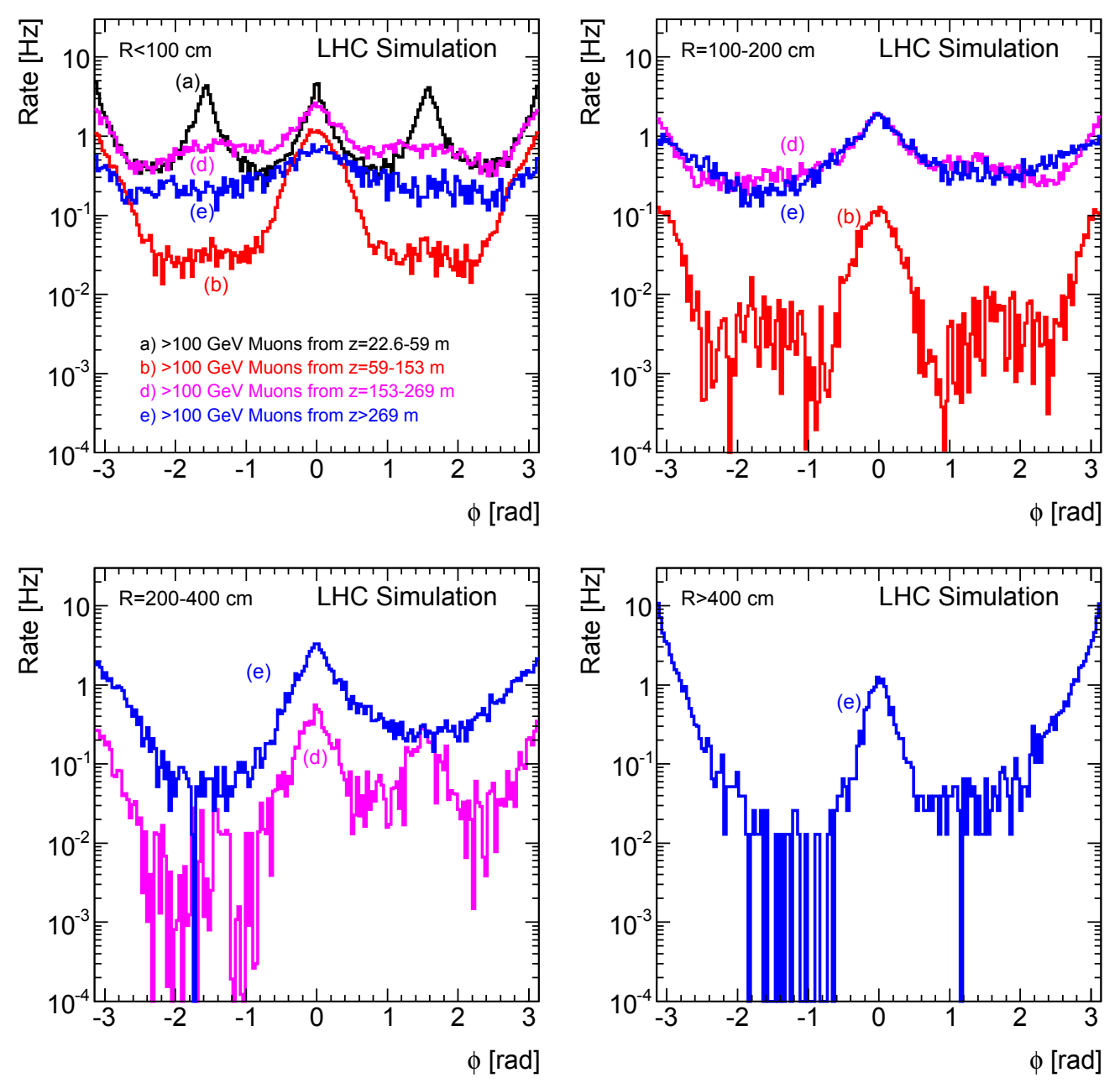

Figure 7. Simulated azimuthal distribution of beam-gas muons at the interface plane in four different radial ranges. The regions of origin considered are the same as in figure 6 . The values have not been normalised to unit area, but represent the rate over the entire surface - which is different in each plot. The letters refer to the regions indicated in figure 4 . The contribution from nearby regions drops quickly with radius. Histograms with negligible contribution have been suppressed.

mixed. The same two-spiked structure is also seen at larger radii. Beyond $r=2 \mathrm{~m}$ a slight up-down asymmetry is observed, which can be attributed to a non-symmetric position of the beam-line with respect to the tunnel floor and ceiling - depending on the region, the beam-line is about $1 \mathrm{~m}$ above the floor and about $2 \mathrm{~m}$ below the ceiling. This causes a different free drift for upwardand downward-going pions and kaons to decay into muons before interacting in material. Since the floor is closer than the roof, fewer high-energy muons are expected in the lower hemisphere. A similar up-down asymmetry was already observed in calorimetric energy deposition when $450 \mathrm{GeV}$ low-intensity proton bunches were dumped on the TCT during LHC beam commissioning [27], although in this case high-energy muons probably were a small contribution to the total calorimeter energy. Finally, at radii beyond $4 \mathrm{~m}$, only muons from the arc contribute. The peak at $|\phi|=\pi$ is clearly dominant, and is due to the muons being emitted tangentially to the outside of the ring. 


\begin{tabular}{|l|l|l|}
\hline Trigger item & Description & Usage in background studies \\
\hline L1_BCM_AC_CA_BGRP0 & BCM background-like coincidence & BIB level monitoring \\
L1_BCM_AC_CA_UnpairedIso & BCM background-like coincidence & BIB level monitoring \\
L1_BCM_Wide_UnpairedIso & BCM collision-like coincidence & Ghost collisions \\
L1_BCM_Wide_UnpairedNonIso & BCM collision-like coincidence & Ghost collisions \\
L1_J10_UnpairedIso & Jet with $p_{\mathrm{T}}>10 \mathrm{GeV}$ at L1 & Fake jets \& ghost collisions \\
L1_J10_UnpairedNonIso & Jet with $p_{\mathrm{T}}>10 \mathrm{GeV}$ at L1 & Fake jets \& ghost collisions \\
\hline
\end{tabular}

Table 1. ATLAS trigger items used during the 2011 proton runs for background studies and monitoring.

\section{BIB monitoring with Level-1 trigger rates}

The system that provides the Level-1 (L1) trigger decision, the ATLAS Central Trigger Processor (CTP) [28], organises the BCIDs into Bunch Groups (BG) to account for the very different characteristics, trigger rates, and use-cases of colliding, unpaired, and empty bunches. The BGs are adapted to the pattern of each LHC fill and their purpose is to group together BCIDs with similar characteristics as far as trigger rates are concerned. In particular, the same trigger item can have different prescales in different BGs.

The BGs of interest for background studies are:

- BGRP0, all BCIDs, except a few at the end of the abort gap

- Paired, a bunch in both LHC beams in the same BCID

- Unpaired isolated (UnpairedIso), a bunch in only one LHC beam with no bunch in the other beam within \pm 3 BCIDs.

- Unpaired non-isolated (UnpairedNonIso), a bunch in only one LHC beam with a nearby bunch (within three BCIDs) in the other beam.

- Empty, a BCID containing no bunch and separated from any bunch by at least five BCIDs.

The L1 trigger items which were primarily used for background monitoring in the 2011 proton run are summarised in table 1 and explained in the following.

The L1_BCM_AC_CA trigger is defined to select particles travelling parallel to the beam, from side $\mathrm{A}$ to side $\mathrm{C}$ or vice-versa. It requires a background-like coincidence of two hits, defined as one (early) hit in a time window $-6.25 \pm 2.73 \mathrm{~ns}$ before the nominal collision time and the other (in-time) hit in a time window $+6.25 \pm 2.73 \mathrm{~ns}$ after the nominal collision time.

Table 1 lists two types of BCM background-like triggers - one in BGRP0, and the other in the UnpairedIso BG. The motivation to move from L1_BCM_AC_CA_BGRP0, used in 2010 [29], to unpaired bunches was that a study of 2010 data revealed a significant luminosity-related contamination due to accidental background-like coincidences in the trigger on all bunches (BGRP0). Although the time window of the trigger is narrow enough to discriminate collision products from the actually passing bunch, each proton-proton event is followed by afterglow [30], i.e. delayed tails of the particle cascades produced in the detector material. The afterglow in the BCM is exponentially falling and the tail extends to $\sim 10 \mu$ s after the collision. With $50 \mathrm{~ns}$ bunch spacing this afterglow piles up and becomes intense enough to have a non-negligible probability for 
causing an upstream hit in a later BCID that is in background-like coincidence with a true background hit in the downstream detector arm. In the rest of this paper, unless otherwise stated, the L1_BCM_AC_CA_UnpairedIso rate before prescaling is referred to as BCM background rate.

A small fraction of the protons injected into the LHC escape their nominal bunches. If this happens in the injectors, the bunches usually end up in neighbouring RF buckets. If the bunches are within the same $25 \mathrm{~ns}$ BCID as the main bunch, they are referred to as satellites. If de- and rebunching happens during RF capture in the LHC, the protons spread over a wide range of buckets and if they fall outside filled BCIDs, they are referred to as ghost charge.

The L1_BCM_Wide triggers require a collision-like coincidence, i.e. in-time hits on both sides of the IP. The time window to accept hits extends from $0.39 \mathrm{~ns}$ to $8.19 \mathrm{~ns}$ after the nominal collision time.

The L1_J10 triggers fire on an energy deposition above $10 \mathrm{GeV}$, at approximately electromagnetic scale, in the transverse plane in an $\eta-\phi$ region with a width of about $0.8 \times 0.8$ anywhere within $|\eta|<3.0$ and, with reduced efficiency, up to $\eta \mid=3.2$. Like the L1_BCM_Wide triggers, the two L1_J10 triggers given in table 1 are active in UnpairedIso or UnpairedNonIso bunches, which makes them suitable for studies of ghost collisions rates in these two categories of unpaired bunches.

The original motivation for introducing the UnpairedIso BG was to stay clear of this ghost charge, while the UnpairedNonIso BG was intended to be used to estimate the amount of this component. However, as will be shown, an isolation by \pm 3 BCID is not always sufficient, and some of the UnpairedIso bunches still have signs of collision activity. Therefore table 1 lists the UnpairedIso BG as suitable for ghost charge studies.

\subsection{BCM background rates vs residual pressure}

In order to understand the origin of the background seen by the BCM, the evolution of the rates and residual pressure in various parts of the beam pipe at the beginning of an LHC fill are studied. The vacuum gauges providing data for this study are located at $58 \mathrm{~m}, 22 \mathrm{~m}$ and $18 \mathrm{~m}$ from the IP. The pressures from these are referred to as P58, P22 and P18, respectively. Figure 8 shows a characteristic evolution of pressures and BCM background rate when the beams are injected, ramped and brought into collision. P58 starts to increase as soon as beam is injected into the LHC. The pressure, however, does not reflect itself in the background seen by the BCM. Only when the beams are ramped from $450 \mathrm{GeV}$ to $3.5 \mathrm{TeV}$, does $\mathrm{P} 22$ increase, presumably due to increased synchrotron radiation from the inner triplet. The observed BCM background increase is disproportionate to the pressure increase. This is explained by the increasing beam energy, which causes the produced secondary particles, besides being more numerous, to have higher probability for inducing penetrating showers in the TAS, which is between the $22 \mathrm{~m}$ point and the BCM. The pressure of the third gauge, located at $18 \mathrm{~m}$ in a NEG-coated section of the vacuum pipe, is not shown in figure 8. The NEG-coating reduces the pressure by almost two orders of magnitude, such that the residual gas within $\pm 19 \mathrm{~m}$ does not contribute significantly to the background rate. According to figure 4 , the pressure measured by the $22 \mathrm{~m}$ gauge is constant through the entire inner triplet. ${ }^{10}$ This and the correlation with P22 suggest that the background seen by the BCM is due mostly to beam-gas events in the inner triplet region.

\footnotetext{
${ }^{10}$ The pressure simulation is based, among other aspects, on the distribution and intensity of synchrotron radiation, which is assumed to be constant within the triplet.
} 


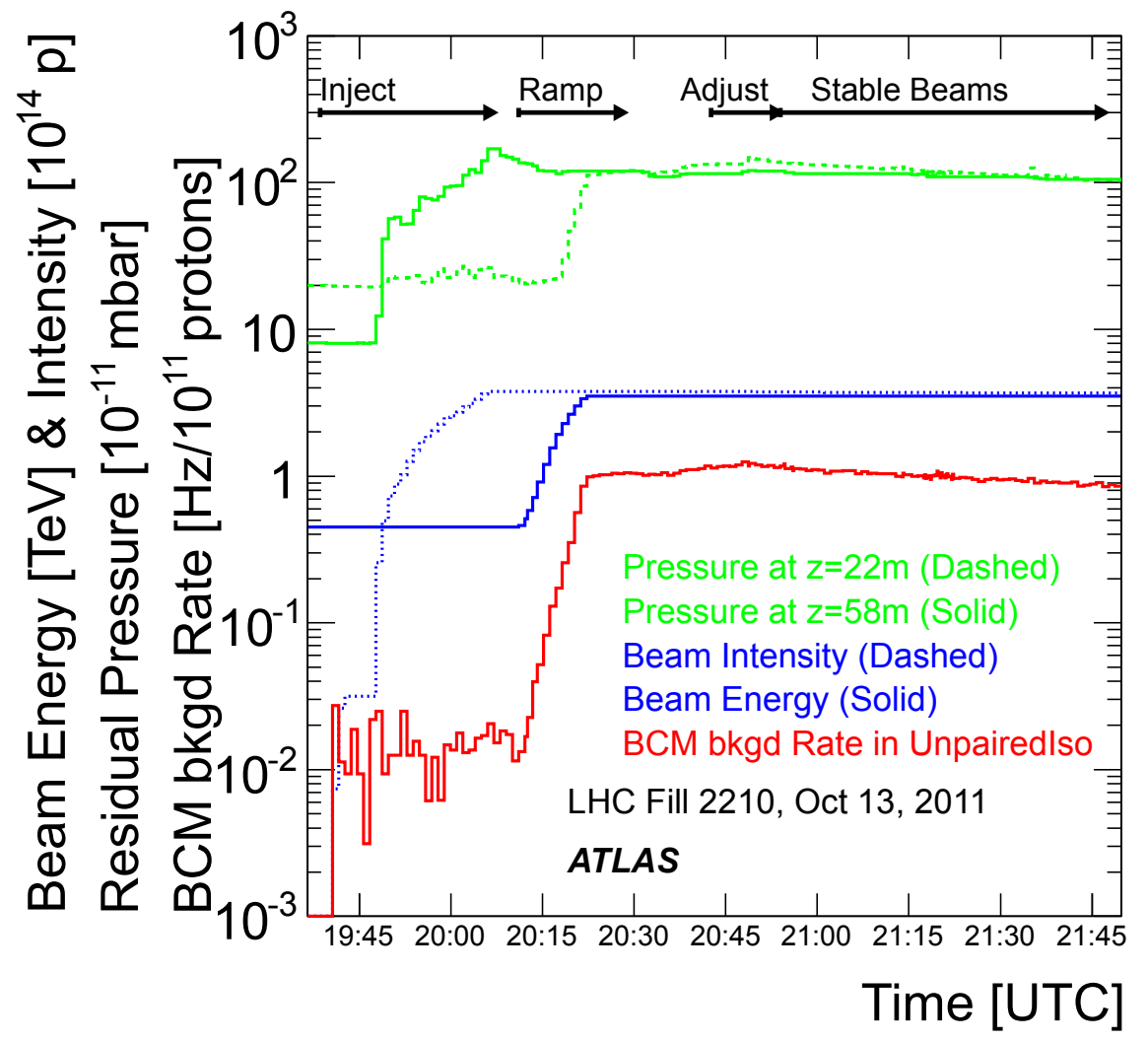

Figure 8. Pressures, beam parameters and BCM background rates during the start of a typical LHC fill.

This conclusion is further supported by figure 9 where the BCM background rate versus $\mathrm{P} 22$ is shown. In the plot each point represents one LB, i.e. about 60 seconds of data-taking. Since beam intensities decay during a fill, the pressures and background rate also decrease so that individual LHC fills are seen in the plot as continuous lines of dots. A clear, although not perfect, correlation can be observed. There are a few outliers with low pressure and relatively high rate. All of these are associated with fills where P58 was abnormally high.

The relative influence of P22 and P58 on the BCM background was studied in a special test, where the small solenoids around the beam pipe at $58 \mathrm{~m}$, intended to suppress electron-cloud formation, were gradually turned off and back on again. Figure 10 shows the results of this study. The solenoids were turned off in three steps and due to the onset of electron-cloud formation the pressure at $58 \mathrm{~m}$ increased by a factor of about 50 . At the same time the pressure at $22 \mathrm{~m}$ showed only the gradual decrease due to intensity lifetime. With the solenoids turned off, P58 was about nine times larger than P22. At the same time the BCM background rate increased by only $30 \%$, while it showed perfect proportionality to P22 when the solenoids were on and P58 suppressed. This allows quantifying the relative effect of P58 on the BCM background to be about $3-4 \%$ of that of P22. If these 3-4\% were taken into account in figure 9, the outliers described above would be almost entirely brought into the main distribution.

In summary, the BCM background trigger can be considered to be a very good measurement of beam-gas rate produced close to the experiment, while it has low efficiency to monitor beam losses far away from the detector. 


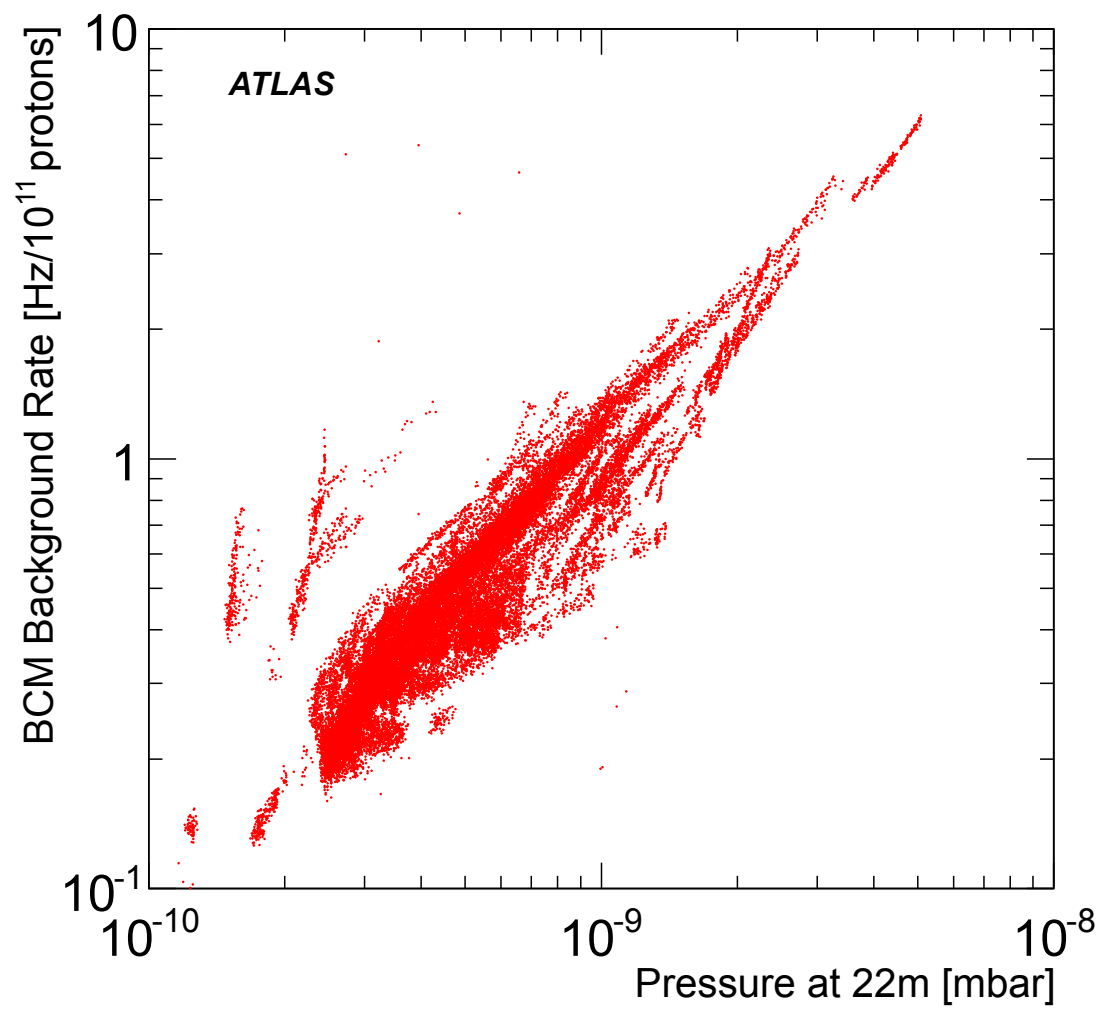

Figure 9. Correlation between P22 and BCM background rate. Each dot represents one LB.

\subsection{BCM background rates during 2011}

Figure 11 shows the $\mathrm{BCM}$ background rate for the 2011 proton runs together with the $\mathrm{P} 22$ average residual pressure. These rates are based on the L1_BCM_AC_CA_UnpairedIso trigger rates, which became available after the May technical stop of the LHC. During the period covered by the plot, the number of unpaired bunches and their location in the fill pattern changed considerably. No obvious correlation between the scatter of the data and these changes could be identified. No particular time structure or long-term trend can be observed in the 2011 data. The average value of the intensity-normalised rate remains just below $1 \mathrm{~Hz}$ throughout the year.

Except for a few outliers, due to abnormally high P58, the BCM background rate correlates well with the average P22 residual pressure, in agreement with figure 9 and the discussion in section 5.1.

\subsection{Observation of ghost charge}

The BCM allows studies of the amount of ghost charge in nominally empty BCIDs. The background-like trigger can be used to select beam-gas events created by ghost charge. Since, for a given pressure, the beam-gas event rate is a function of bunch intensity only, this trigger yields directly the relative intensity of the ghost charge with respect to a nominal bunch, in principle. The rate, however, is small and almost entirely absorbed in backgrounds, mainly the accidental afterglow coincidences discussed at the beginning of this section. Another problem is that due to the width of the background trigger time window, only the charge in two or three RF buckets is seen, depending on how accurately the window is centred around the nominal collision time. 


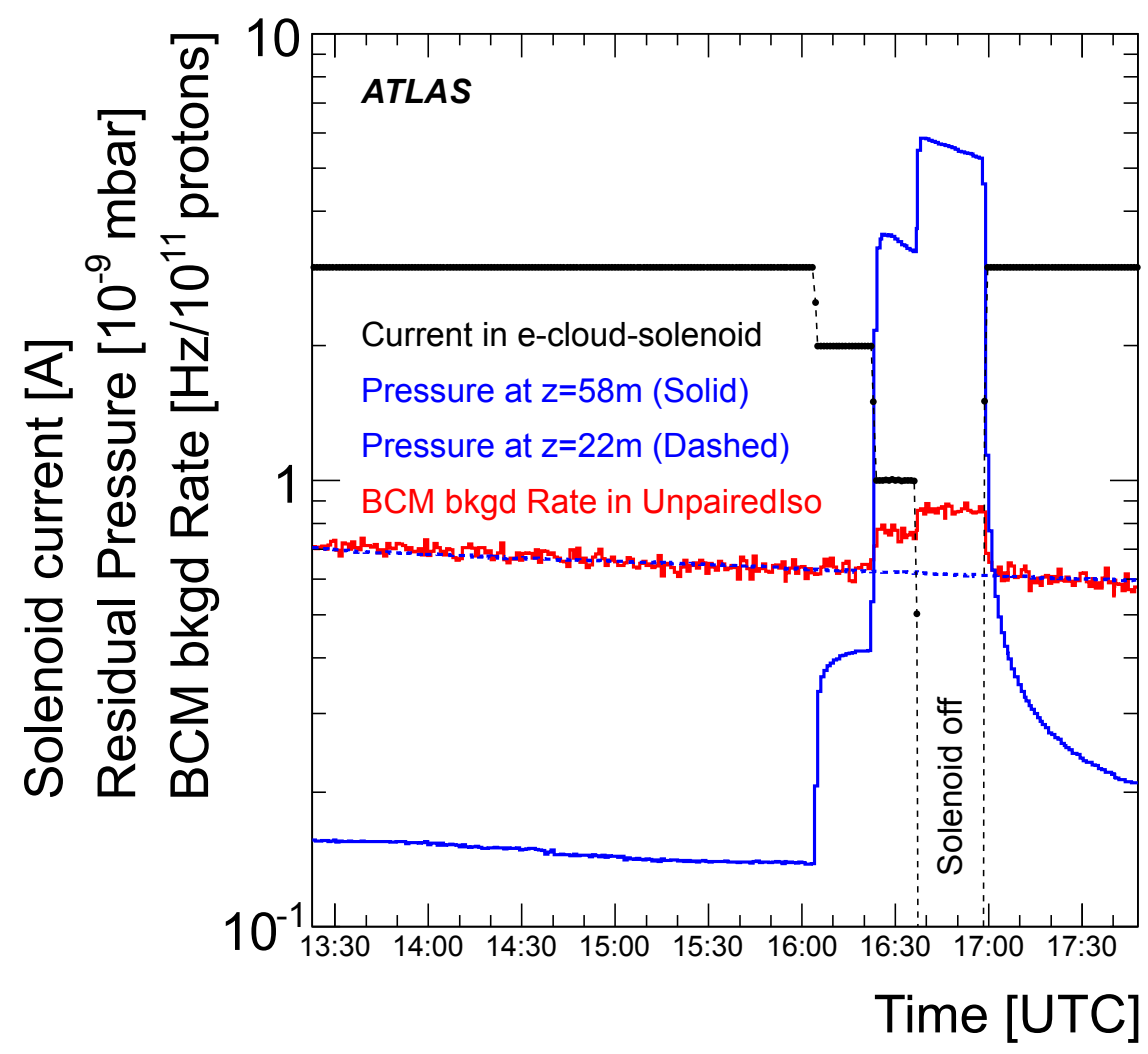

Figure 10. P58, P22 and background seen at the BCM during the "solenoid test" in LHC fill 1803. The dashed line is not a fit, but the actual P22 value, which just happens to agree numerically with the BCM rate on this scale.

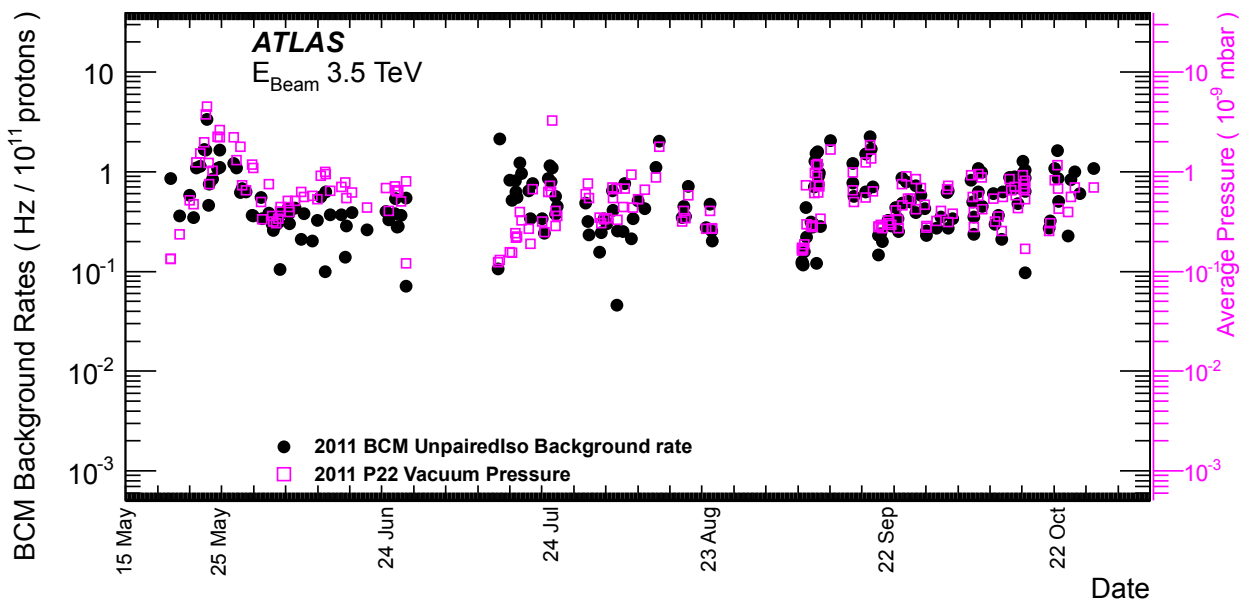

Figure 11. BCM background rate normalised to $10^{11}$ protons for the 2011 proton-proton running period starting from mid-May. The rate is shown together with the P22 average residual pressure. 
A more sensitive method is to look at the collisions of a ghost bunch with nominal bunches. Provided the emittance of the ghost bunches is the same as that of nominal ones, the luminosity of these collisions, relative to normal per-bunch luminosity gives directly the fraction of ghost charge in the bucket with respect to a nominal bunch. The collisions probe the ghost charge only in the nominal RF bucket, which is the only one colliding with the unpaired bunch. The charge in the other nine RF buckets of the BCID is not seen. Data from the Longitudinal Density Monitors of the LHC indicate that the ghost charge is quite uniformly distributed in all RF buckets of a non-colliding BCID [31, 32].

Figure 12 shows a summary of BCM collision-like and background-like trigger rates for a particularly interesting BCID range of a bunch pattern with 1317 colliding bunches. For this plot, several ATLAS runs with the same bunch-pattern and comparable initial beam intensities have been averaged. The first train of a batch is shown with part of the second train. The symbols show the trigger rates with both beams at $3.5 \mathrm{TeV}$ but before they are brought into collision, while the histograms show the rates for the first $\sim 15$ minutes of stable beam collisions. This restriction to the start of collisions is necessary since the rates are not normalised by intensity, and a longer period would have biased the histograms due to intensity decay. The groups of six unpaired bunches each in front of the beam-2 trains (around BCID 1700 and 1780, respectively) and after the beam-1 train (around BCID 1770) can be clearly seen. These show the same background trigger rate before and during collisions. As soon as the beams collide, the collision rate in paired BCIDs rises, but the background rate also increases by about an order of magnitude. As explained before, this increase is due to accidental background-like coincidences from afterglow. The gradual build-up of this excess is typical of afterglow build-up within the train [30].

The uppermost plot in figure 12, showing the collision rate, reveals two interesting features:

- Collision activity can be clearly seen in front of the train, in BCIDs 1701, 1703 and 1705. This correlates with slightly increased background seen in the middle plot for the same BCIDs. This slight excess seen both before and during collisions is indicative of ghost charge and since there are nominal unpaired bunches in beam- 2 in the matching BCIDs, this results in genuine collisions. It is worth noting that a similar excess does not appear in front of the second train of the batch, seen on the very right in the plots. This is consistent with no beam-1 ghost charge being visible in the middle plot around BCID 1780 .

- Another interesting feature is seen around BCID 1775, where a small peak is seen in the collision rate. This peak correlates with a BCID range where beam-1 bunches are in odd BCIDs and beam- 2 in even BCIDs. Thus the bunches are interleaved with only $25 \mathrm{~ns}$ spacing. Therefore this peak is almost certainly due to ghost charge in the neighbouring BCID, colliding with the nominal bunch in the other beam.

The two features described above are not restricted to single LHC fills, but appear rather consistently in all fills with the same bunch pattern. Thus it seems reasonable to assume that this ghost charge distribution is systematically produced in the injectors or RF capture in the LHC.

Figure 12 suggests that the definition of an isolated bunch, used by ATLAS in 2011, is not sufficient to suppress all collision activity. Instead of requiring nothing in the other beam within 

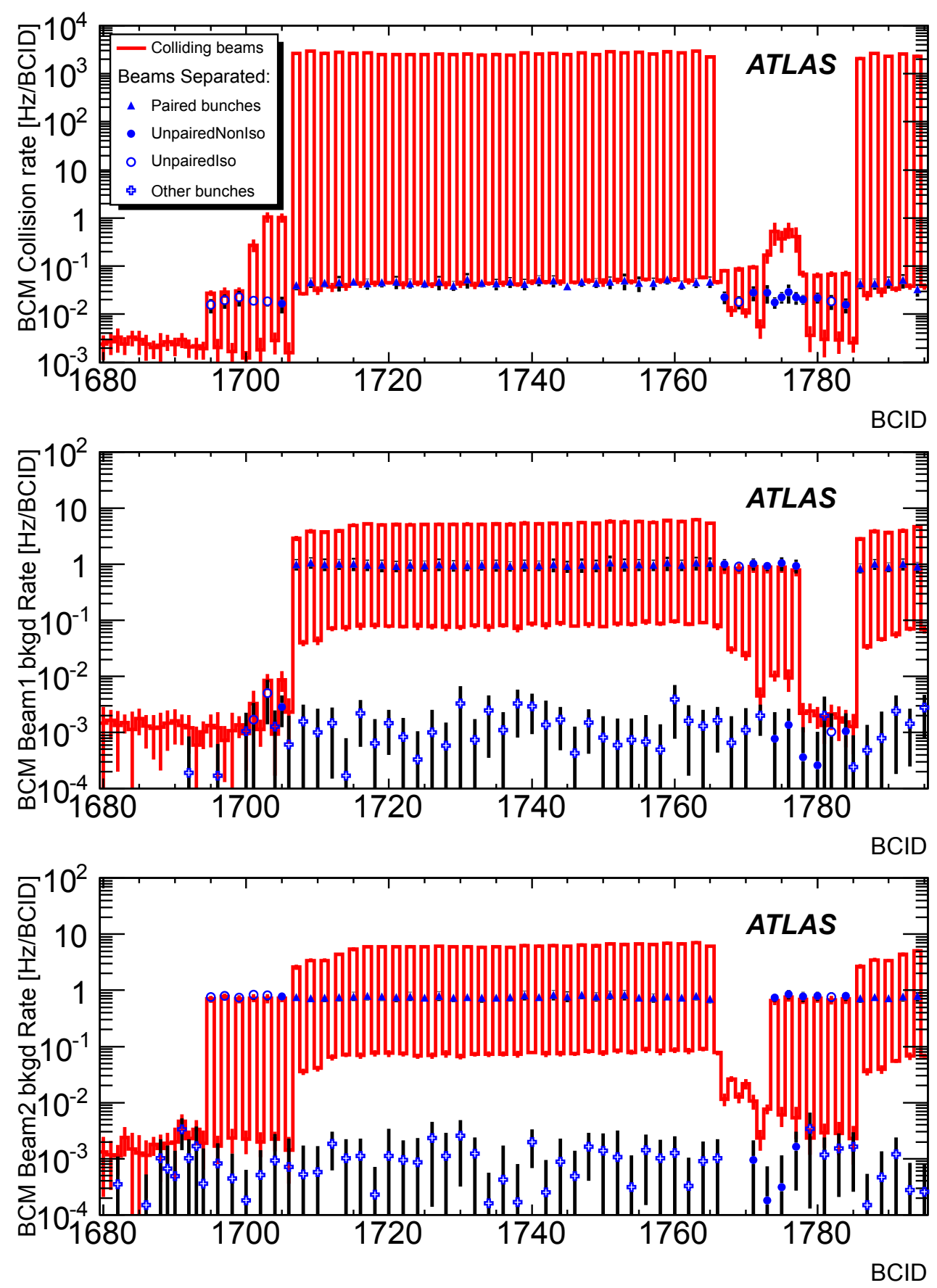

Figure 12. BCM collision rate (top) and background rates for beam-1 (middle) and beam-2 (bottom) per BCID before and during collisions. The data are averaged for several LHC fills over roughly 15 minute periods: at full energy but before bringing beams into collision (symbols) and after declaring stable beams (histogram). Thus the error bars reflect both the fill-to-fill variation and differences of the intensity decay during the averaging time. The data are not normalised by intensity, but only fills with comparable luminosities at the start of the fill are used in the average. 


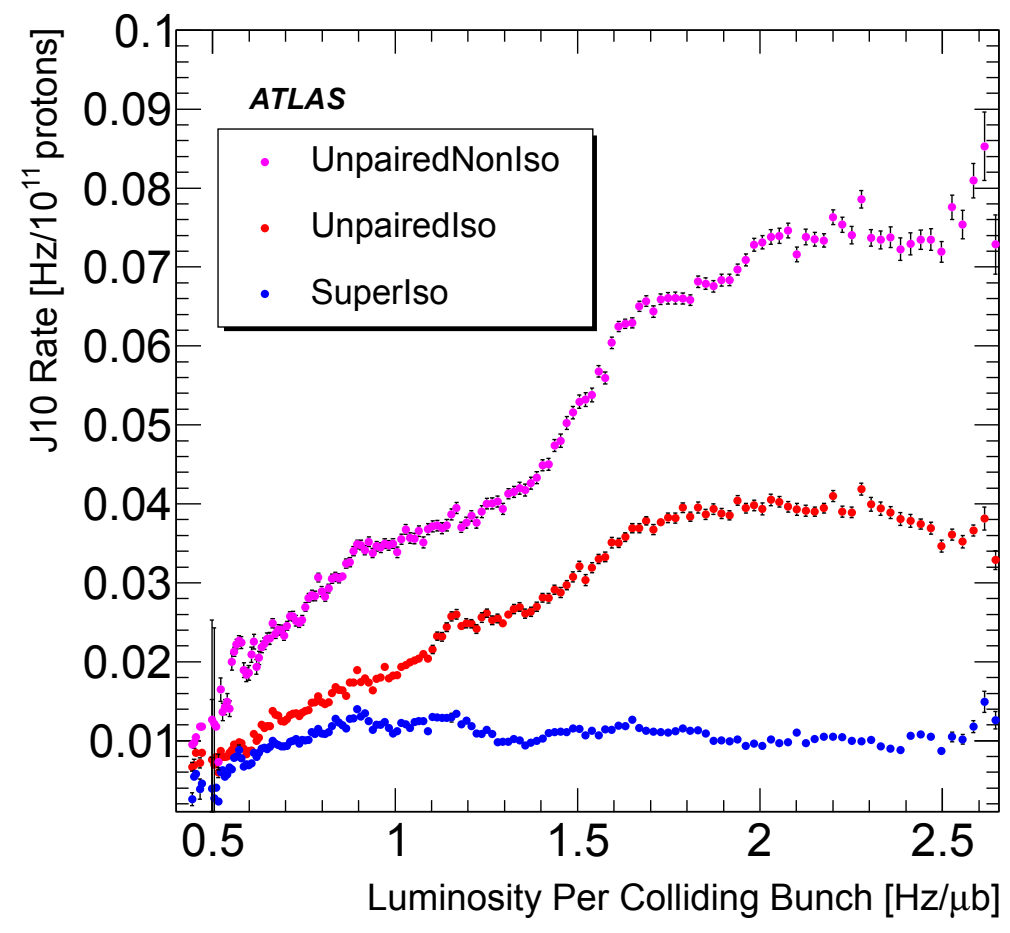

Figure 13. L1_J10 trigger rate in different classes of unpaired bunch isolation as a function of the luminosity of colliding bunches after subtraction of the luminosity-dependent pedestal, determined from the empty bunches.

\pm 3 BCIDs, a better definition would be to require an isolation by \pm 7 BCIDs. In the rest of this paper, bunches with such stronger isolation are called super-isolated (SuperIso). ${ }^{11}$

\subsection{Jet trigger rates in unpaired bunches}

The L1_J10_UnpairedIso trigger listed in table 1 is in principle a suitable trigger to monitor fake-jet rates due to BIB muons. Unfortunately the L1_J10 trigger rate has a large noise component due to a limited number of calorimeter channels which may be affected by a large source of instrumental noise for a short period of time, on the order of seconds or minutes. While these noisy channels are relatively easy to deal with offline by considering the pulse shape of the signal, this is not possible at trigger level. In this study, done on the trigger rates alone, the fluctuations caused by these noise bursts are reduced by rejecting LBs where the intensity-normalised rate is more than $50 \%$ higher than the 5-minute average.

Another feature of the J10 trigger is that the rates show a dependence on the total luminosity even in the empty bunches, i.e. there is a luminosity-dependent constant pedestal in all BCIDs. While this level is insignificant with respect to the rate in colliding BCIDs, it is a nonnegligible fraction of the rates in the unpaired bunches. To remove this effect the rate in the empty BCIDs is averaged in each LB separately and this pedestal is subtracted from the rates in the unpaired bunches.

\footnotetext{
${ }^{11}$ For the start of 2012 data-taking the UnpairedIso BG was redefined to match this definition of SuperIso.
} 

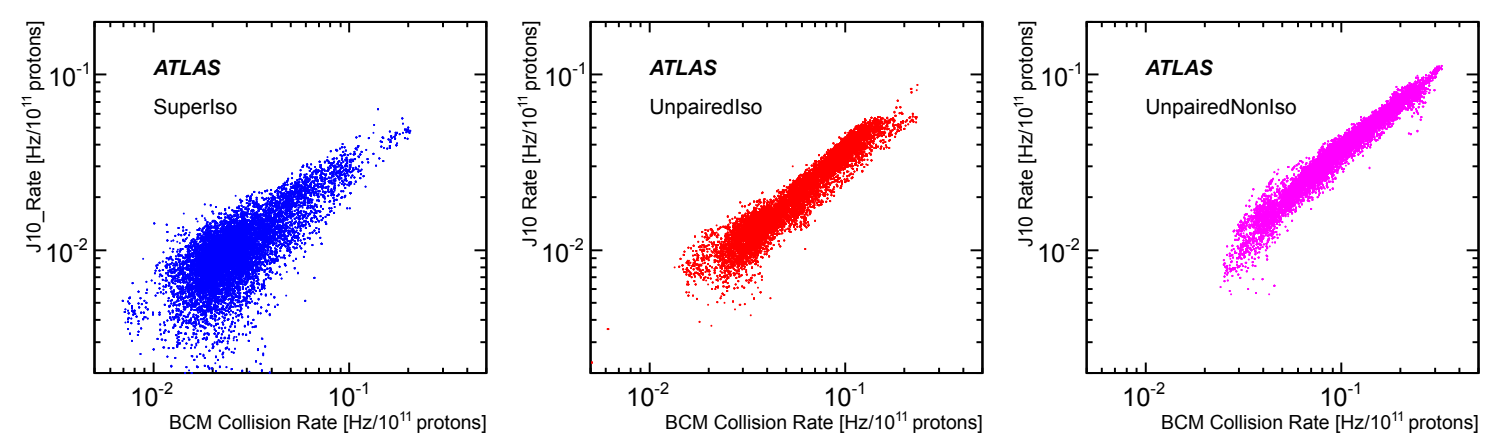

Figure 14. Correlation of L1_J10 and BCM collision trigger rates in different classes of unpaired bunch isolation.

Figure 13 shows these pedestal-subtracted L1_J10 trigger rates in unpaired bunches, plotted against the luminosity of colliding bunches. Provided the intensity of ghost bunches is proportional to the nominal ones, their emittance is the same as that of normal bunches and if all the rate is due to proton-proton collisions, a good correlation is expected. Indeed, the UnpairedNonIso rates correlate rather well with the luminosity, indicating that a large fraction of the rate is due to bunchghost encounters. Even the UnpairedIso rates show some correlation, especially at low luminosity. This suggests that even these isolated bunches are paired with some charge in the other beam which is consistent with figure 12. In superIso bunches, i.e. applying an even tighter isolation, the correlation mostly disappears and the rate is largely independent of luminosity.

If the rates shown in figure 13 are dominated by collisions, then this should be reflected as a good correlation between the J10 and BCM collision-like trigger rates. Figure 14 shows that this is, indeed, the case. While the correlation is rather weak for the superIso bunches, it becomes increasingly stronger with reduced isolation criteria.

\section{Studies of BIB with the ATLAS Pixel detector}

\subsection{Introduction}

Like the BCM, the ATLAS Pixel detector is very close to the beam-line, so it is sensitive to similar background events. However, while the BCM consists of only eight active elements, the Pixel detector has over 80 million read-out channels, each corresponding to at least one pixel. This fine granularity enables a much more detailed study of the characteristics of the BIB events.

As shown in section 5, the BCM background rate is dominated by beam-gas events in rather close proximity to ATLAS. Energetic secondary particles from beam-gas events are likely to impinge on the TAS and initiate showers. The particles emerging from the TAS towards the Pixel detector are essentially parallel to the beam-line and therefore typically hit only individual pixels in each endcap layer, but potentially leave long continuous tracks in Pixel barrel sensors. If a beamgas event takes place very close to the TAS, it is geometrically possible for secondary particles to pass through the aperture and still hit the inner Pixel layer.

In studies using 2010 data [29] the characteristic features of high cluster multiplicity and the presence of long clusters in the $z$-direction in the barrel, were found to be a good indicator of background contamination in collision events. 


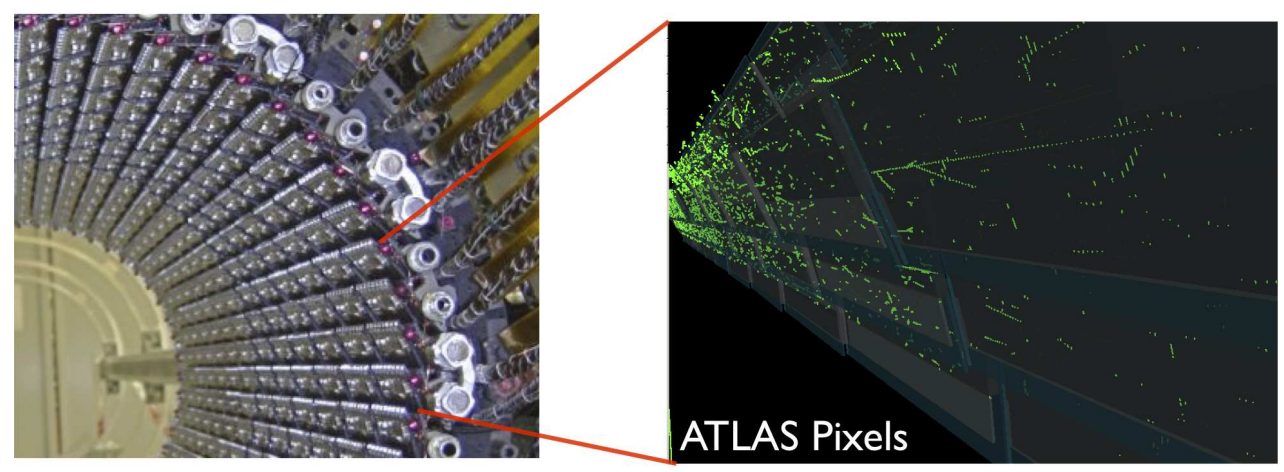

Figure 15. A high-multiplicity BIB event in the Pixel detector, showing the typically long pixel clusters deposited in the barrel region. On the left is the layout of the Pixel detector barrel viewed along the beamline and the right shows the event display in a zoomed region.

The study in ref. [29] was done by considering paired and unpaired BCIDs separately. Comparing the hit multiplicity distributions for these two samples allows the differences between BIB and collision events to be characterised. An independent method to identify BIB events is to use the early arrival time on the upstream side of the detector. While the time difference expected from the half length of the Pixel detector is too short to apply this method with the pixel timing alone, correlations with events selected by other, larger, ATLAS sub-detectors with nanosecond-level time resolution are observed. For example, BIB events identified by a significant time difference between the BCM stations on either side of ATLAS, are also found to exhibit large cluster multiplicity in the Pixel detector [29].

The characterisation of BIB-like events by comparing distributions for paired and unpaired bunches, coupled with the event timing in other sub-detectors, allows parameters to be determined for the efficient identification of BIB in the Pixel detector. The most striking feature in the Pixel barrel of BIB-like events, compared to collision products, is the shallow angle of incidence, which causes Pixel clusters to be elongated along $z$, where a cluster is defined as a group of neighbouring pixels in which charge is deposited. Since the pixels have a length of $400 \mu \mathrm{m}$, or larger, in the $z$-direction, the charge per pixel tends to be larger than for a particle with normal incidence on the $250 \mu \mathrm{m}$ thick sensor. More significantly however, a horizontal track is likely to hit many pixels causing the total cluster charge to be much larger than for typical "collision" clusters.

In the following, the different properties of pixel clusters generated by collisions and BIB events are examined to help develop a background identification algorithm, which relies only on the cluster properties. The BIB tagging efficiency is quantified and the tools are applied to study 2011 data.

\subsection{Pixel cluster properties}

An example of a high-multiplicity BIB event is shown in figure 15, in which the elongated clusters in the barrel region can be observed.

The differences in average cluster properties for collision-like and BIB-like events are shown in figure 16. For each barrel layer and endcap, the pixel cluster column width in the $\eta$ direction is averaged over all clusters and plotted against the pseudorapidity of the cluster position. Ganged pixels are excluded and no requirement for the clusters to be associated with a track is applied. 


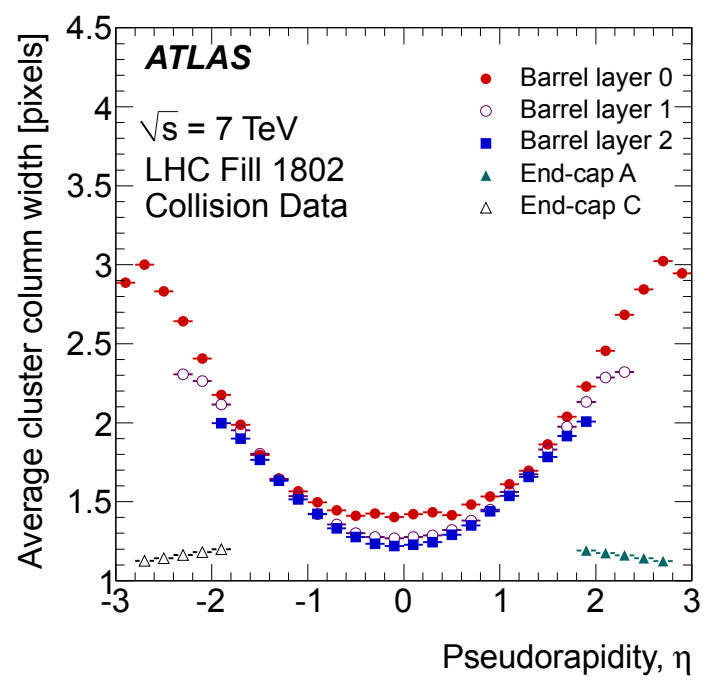

(a)

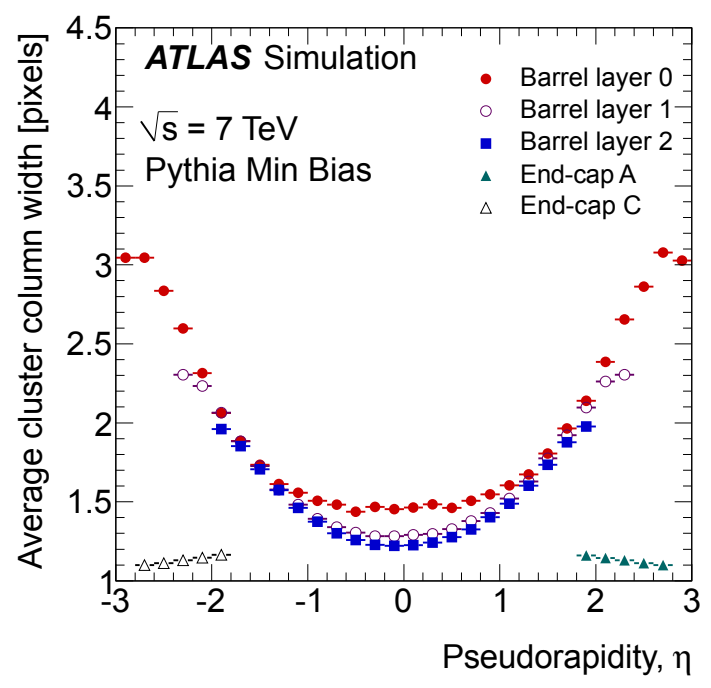

(c)

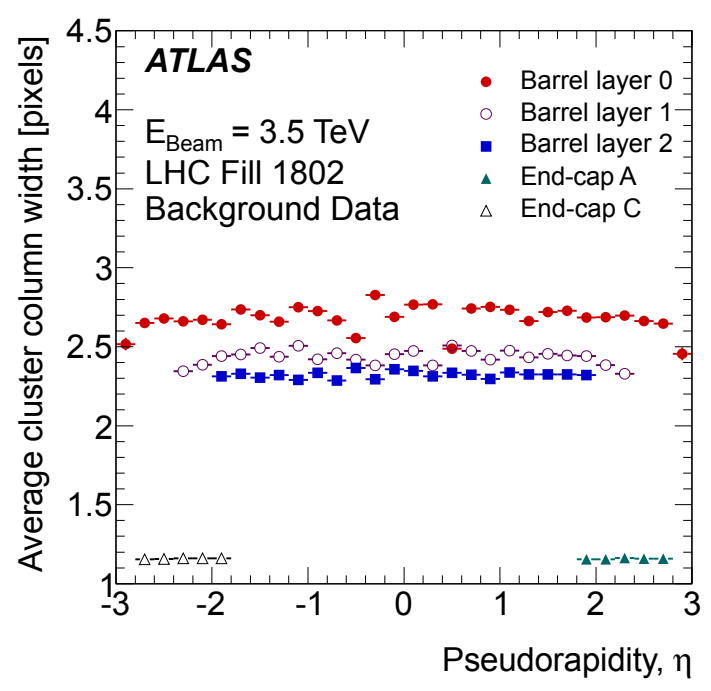

(b)

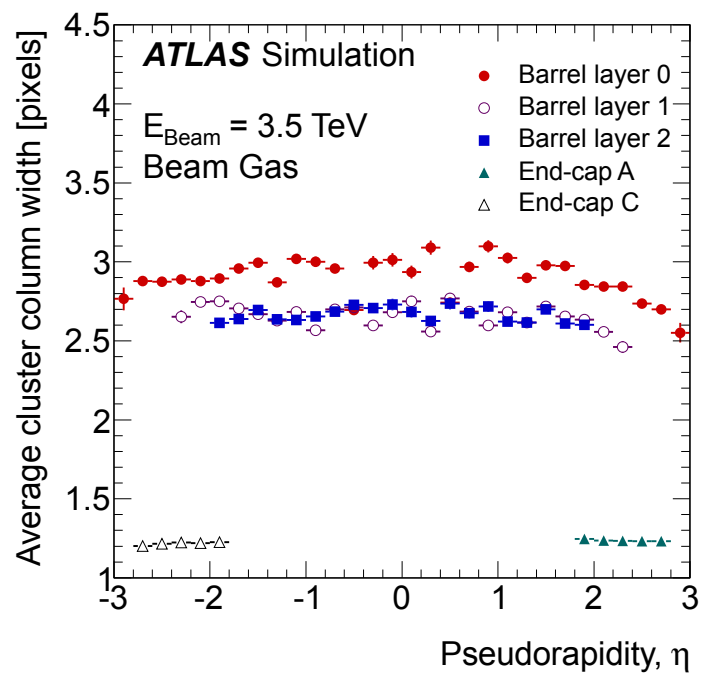

(d)

Figure 16. Pixel cluster width (in $\eta$ direction) versus pseudorapidity for (a, c) collision and (b, d) background data and Monte Carlo simulation.

For collisions, shown on the left of figure 16, the cluster width is a function of $\eta$ simply for geometrical reasons and the agreement between data and Monte Carlo simulation [33] is good.

The distribution for BIB-like events is shown on the right side of figure 16. The upper plot shows data in super-isolated unpaired bunches for events that are selected using the background identification tool, which is described in section 6.3. The distribution is independent of $\eta$ as expected for BIB tracks. A detailed simulation [9], described in section 4, was interfaced to the ATLAS detector simulation to check the cluster properties in beam-gas events. Based on the assumption that BIB in the detector is dominated by showering in the TAS, a $20 \mathrm{GeV}$ energy transport 
cut was used in the beam-gas simulations. This high cut allowed maximisation of the statistics by discarding particles that would not have enough energy to penetrate the $1.8 \mathrm{~m}$ of copper of the TAS. Here it is assumed that particles passing through the TAS aperture, which might have low energy, do not change the average cluster properties significantly - an assumption that remains to be verified by further, more detailed, simulations. The distributions are found to match very well the distributions observed in data. It can also be seen from figure 16 that the clusters in the endcaps are small and of comparable size for both collision events and BIB. This is expected from the geometry, because at the $\eta$-values covered by the endcap disks, the collision products have a very small angle with respect to the beam-line. In the Barrel, layer 0 clusters are systematically larger than layer 1 and layer 2 for small $\eta$, due to the beam spot spread along the beam-line.

In the Pixel detector, the charge deposited in each pixel is measured from the time that the signal is above the discriminator threshold. After appropriate calibration, the charge is determined and summed over all pixels in the cluster. Figure 17 shows the charge versus the cluster column width for the outer barrel layer for the same data and Monte Carlo samples that are used for figure 16. As expected, the majority of clusters are small both in terms of spatial extent and amount of charge.

However, differences between BIB and collision samples become apparent when clusters of larger size or charge are considered. In the BIB events, a strong correlation is observed between cluster width and deposited charge, because the elongated clusters tend to align along the beam direction. Large clusters in collision events, however, may arise either from secondary particles such as $\delta$-rays or low-momentum loopers, or from particles stopping in the sensor (Bragg-peak). Thus the clusters with large charges are not necessarily aligned with the beam direction. These features, seen in data, are qualitatively well reproduced by the Monte Carlo simulations.

\subsection{Pixel cluster compatibility method}

The cluster characteristics of BIB particles have been exploited to develop a BIB identification algorithm, based on a check of the compatibility of the pixel cluster shape with BIB.

Only the cluster widths, $\Delta \eta$ and $\Delta \phi$, are necessary for an efficient selection of BIB. The algorithm processes all clusters in the event, independent of whether the cluster is associated with a track after reconstruction. Therefore, in addition to offline analysis, the algorithm is also suited for rapid online monitoring of the background.

For each pixel cluster in the event, the algorithm computes the conditional probability to obtain the measured cluster width, $w=\Delta \eta$ (or $\Delta \phi$ ), (in units of pixels), given the cluster position in pseudorapidity, $\eta$, and the barrel layer. Only pixel clusters in the barrel layers are considered, as these provide the best discriminating power. The conditional probability associated with each possible source of the cluster, $P^{c}$ for collisions or $P^{b}$ for BIB, is retrieved from look-up tables, $T_{w}^{c}$ for collisions or $T_{w}^{b}$ for BIB:

$$
P^{c, b}(w \mid \eta, \text { layer })=\frac{T_{w}^{c, b}(\eta, \text { layer })}{\sum_{w=1}^{N} T_{w}^{c, b}(\eta, \text { layer })}
$$

where $T_{w}^{c, b}(\eta$,layer $)$ is the number of clusters with width $w$ for a given $\eta$ bin and barrel layer.

The values for $T_{w}^{c, b}$ were obtained using a data-driven method based on studies of colliding and unpaired bunches. The study was performed using LHC fill 1022 from 2010, in which the bunch 


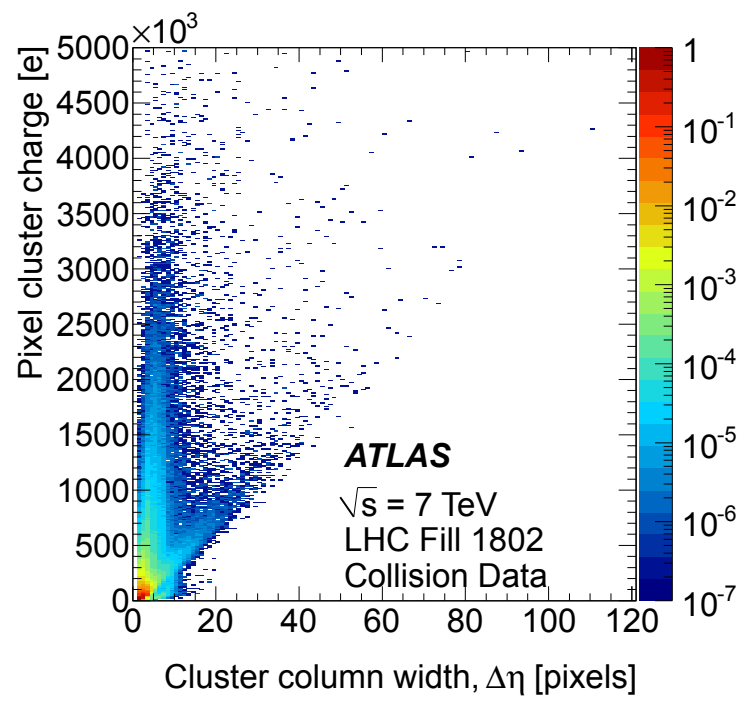

(a)

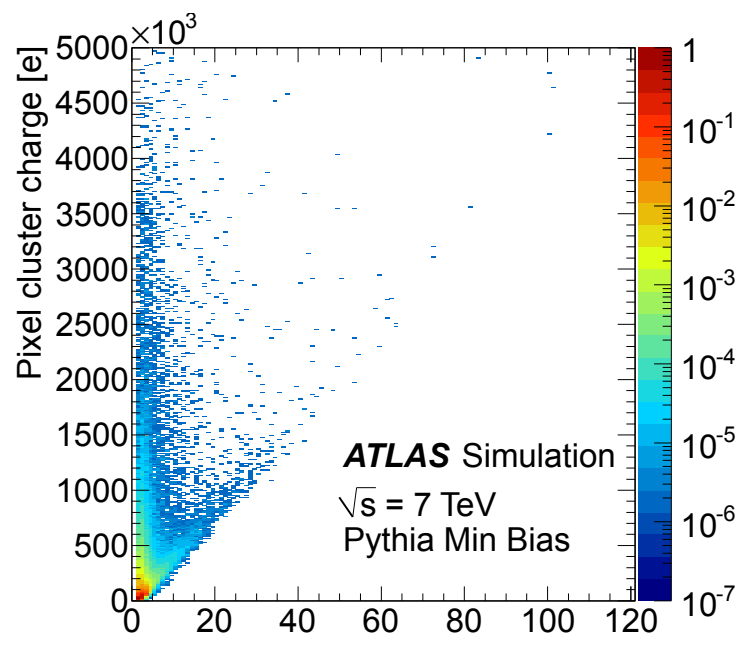

Cluster column width, $\Delta \eta$ [pixels]

(c)

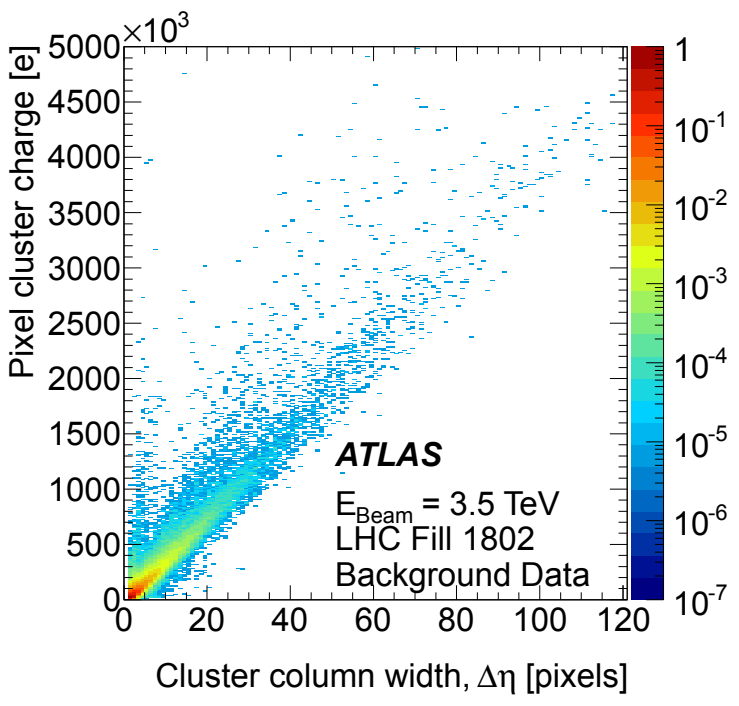

(b)

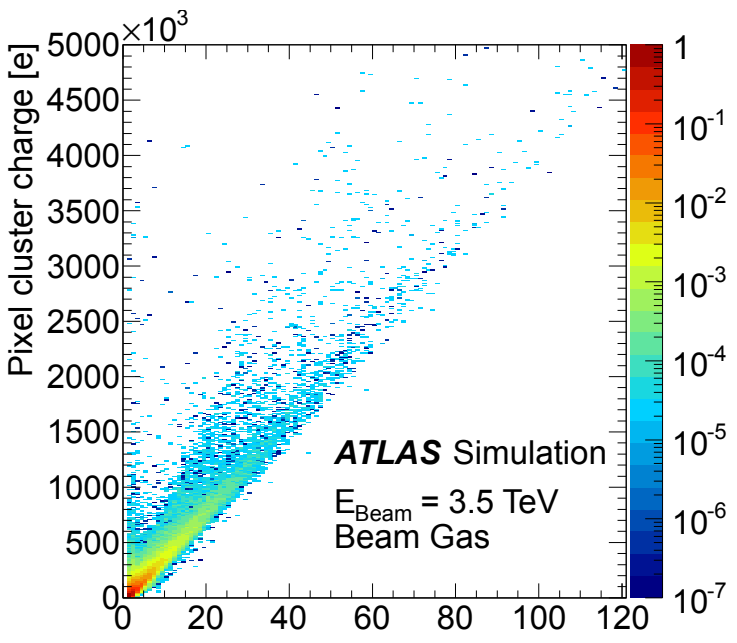

Cluster column width, $\Delta \eta$ [pixels]

(d)

Figure 17. Pixel cluster deposited charge versus cluster width (in $\eta$ direction), for Pixel barrel clusters.

configuration had only one pair of colliding bunches in BCID $=1$ and one unpaired bunch per beam, in BCID 892 and 1786. In this sparse pattern, the unpaired bunches satisfied the definition of being super-isolated.

The conditional probability distribution for pixel clusters in the innermost barrel layer is plotted in figure 18, for different cluster widths and for colliding, $P^{c}$ (left), and unpaired, $P^{b}$ (right), bunches. The probability distributions are shown for the cluster width in the $\eta$ direction only. The other barrel layers have similar distributions, with reduced pseudorapidity coverage. As described by eq. (6.1), the pixel cluster width distributions are normalised to the total number of pixel clusters in each pseudorapidity bin, so that the relative multiplicity of all cluster widths can 


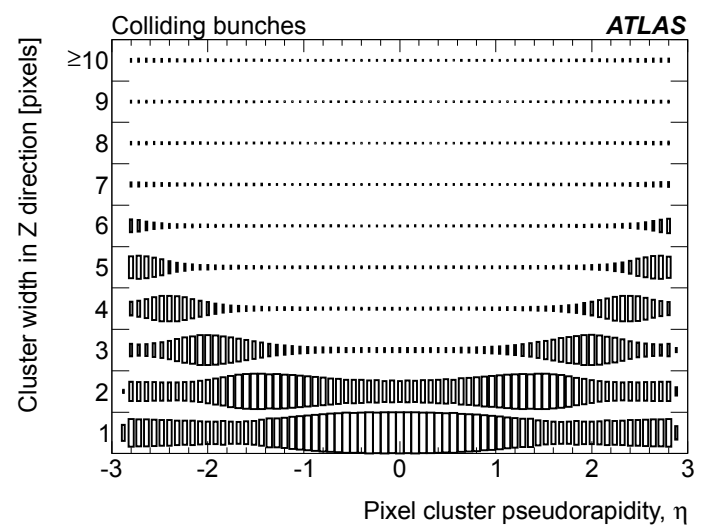

(a)

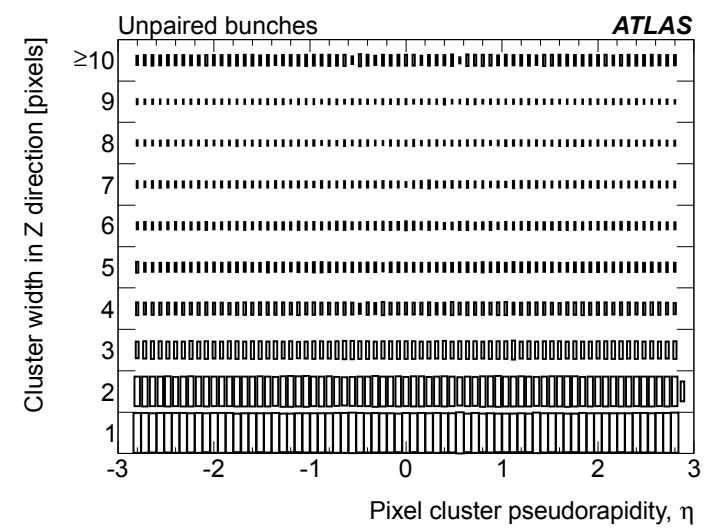

(b)

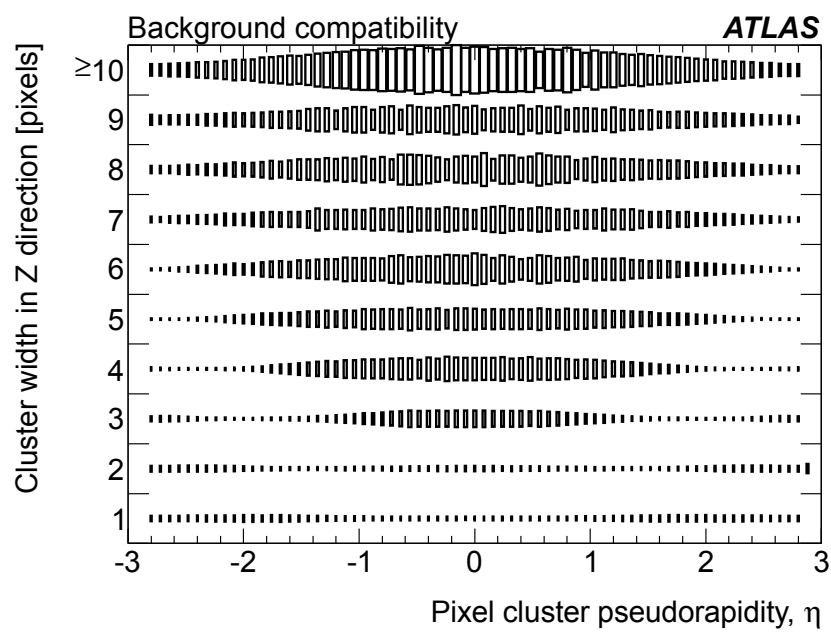

(c)

Figure 18. Conditional probability distributions, $P^{c}$ and $P^{b}$, for clusters in the innermost pixel barrel for (a) colliding and (b) unpaired bunches respectively. The lower plot (c) shows the calculated BIB compatibility.

be compared. It is seen that the fraction of clusters with a certain width depends strongly on $\eta$ for colliding bunches, whereas the probability to generate a certain width of cluster is independent of $\eta$ for clusters from BIB.

The conditional probability distributions are used to construct the compatibility of the cluster with BIB rather than with collisions. The BIB compatibility $C^{b}$, is defined as the ratio of conditional probabilities, and is calculated independently for the cluster $w=\Delta \eta$ and $w=\Delta \phi$ dimensions:

$$
C^{b}(w \mid \eta, \text { layer })=\frac{P^{b}(w \mid \eta, \text { layer })}{P^{c}(w \mid \eta, \text { layer })}
$$

The resulting BIB compatibility is plotted in figure 18(c) and has the expected distribution; the longest pixel clusters in the central barrel region are the best indicators of BIB. Similar plots are obtained for the cluster widths in the orthogonal, $\phi$, direction, and both directions are exploited to calculate the background compatibility of the cluster. 


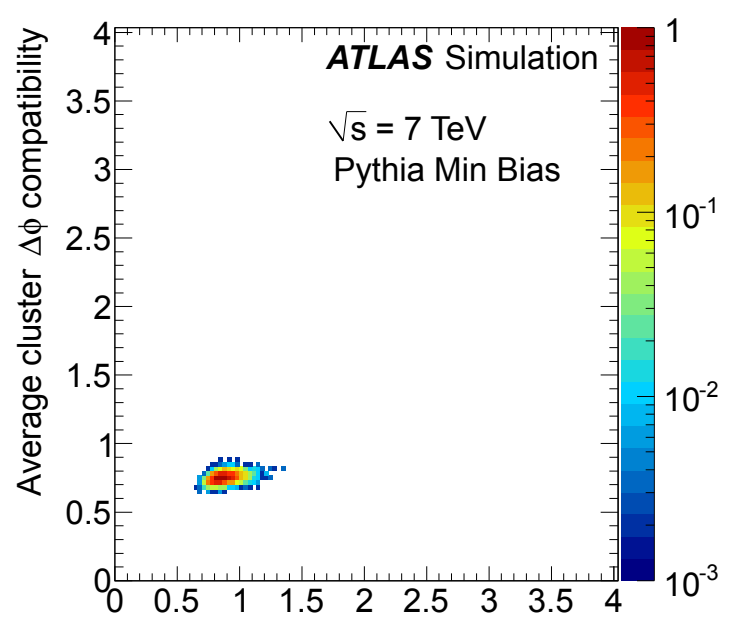

Average cluster $\Delta \eta$ compatibility

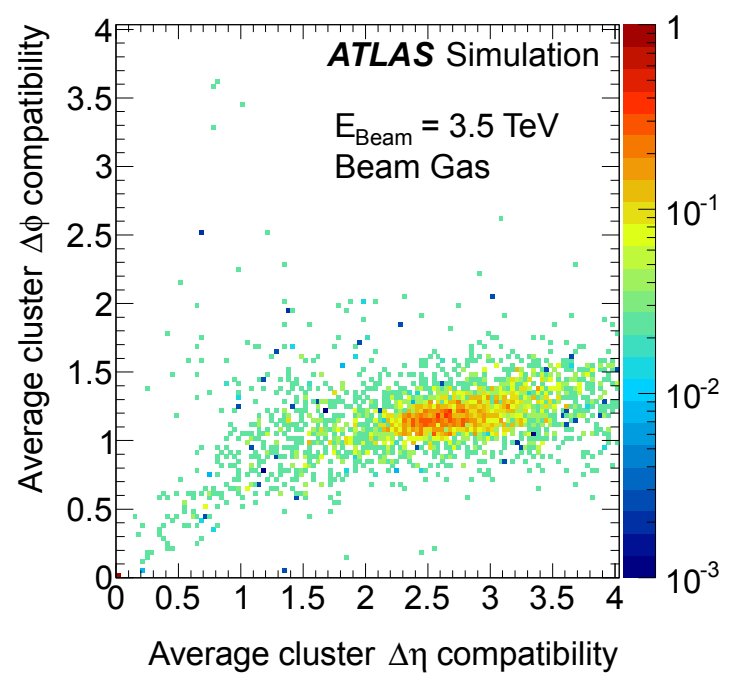

(b)

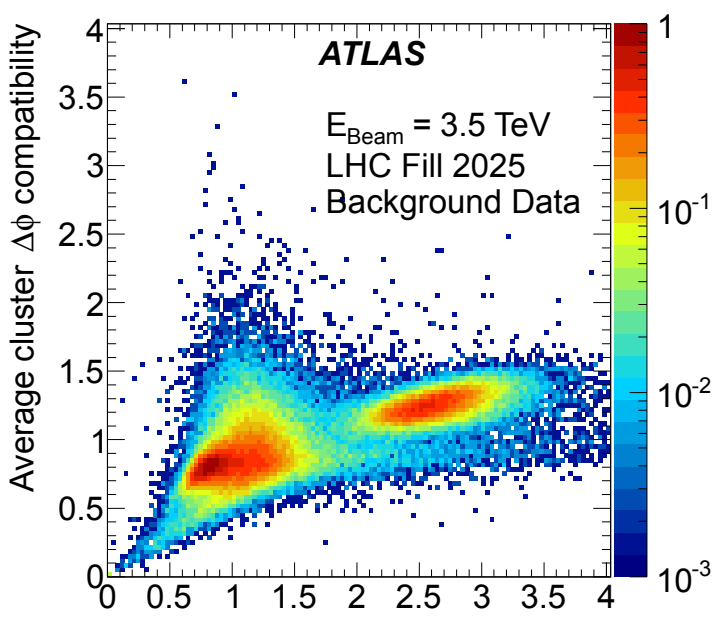

Average cluster $\Delta \eta$ compatibility

(c)

Figure 19. The average pixel cluster compatibility distributions for (a) simulated collisions, (b) simulated beam-gas, and (c) background data. The intensity scale represents the number of events normalised by the maximum bin.

After the compatibility is computed for each cluster in the event, the algorithm uses two methods to identify events containing BIB:

- Simple counting method. In the first method, each pixel cluster is taken to be compatible with BIB if the cluster compatibility in both dimensions exceeds the quality cuts $C^{b}(\Delta \eta \mid \eta$, layer $)>20$ and $C^{b}(\Delta \phi \mid \eta$, layer $)>4$. The entire event is tagged as a BIB candidate if it contains more than five BIB compatible clusters. The quality cuts are tuned in Monte Carlo simulation to efficiently select BIB events, while rejecting collisions. 
- Cluster compatibility averaging. In the second method, the cluster compatibilities in $\Delta \eta$ and $\Delta \phi$ are independently averaged over all clusters in the event. A two-dimensional compatibility distribution is obtained, shown in figures 19(a), 19(b) and 19(c) respectively, for simulated collision events, simulated beam-gas events and a 2011 data run. It is seen from the Monte Carlo samples that the collision and BIB distributions are centred in different regions of the compatibility parameter space. The two regions remain distinct in the background data sample, one corresponding to the unpaired bunch colliding with ghost charge, as discussed in section 5.3, and possibly afterglow, while the other region is dominated by beam-background events. A two-dimensional cut is applied to select BIB candidates.

The simple counting method essentially relies on a sufficient number $(>5)$ of large BIB clusters in the central barrel regions to identify BIB events. The cluster compatibility averaging method takes into account all clusters in the event, so it is suitable for identifying events containing fewer large BIB clusters together with many smaller BIB clusters, which may not be tagged by the simple counting method. If a BIB event is overlaid with multiple collisions, the additional collision-like clusters pull the average compatibility for a BIB event toward the centre of the collision distribution. An increase in pile-up therefore reduces the efficiency for tagging a BIB event using only the cluster compatibility averaging method. However, the tagging efficiency of the simple counting method is robust against pile-up, since an event containing a sufficient number of large BIB compatible clusters is always tagged. At high pile-up the merging of collision-like clusters into bigger ones reduces the rejection power for collision events, because merged collision clusters are more likely to be mistaken as originating from BIB - and the merging probability is a function of cluster density, which increases with pile-up. Therefore, the combination of both methods is used in the final algorithm to ensure the best possible efficiency and rejection power over a wide range of conditions, including the number of BIB pixel clusters in the event.

Figures 20(a) and 20(b) show the tagging efficiency in simulated beam-gas events and the mis-tagging rate in simulated collision events, respectively. It should be noted that figure 20(b) is based on an average pile-up of 21 interactions per bunch crossing, which implies that the peak near 3000 clusters corresponds to an average of about 150 clusters in a single event. Figure 20(c) shows the tagged and untagged events in recorded background data, which contain mostly BIB and sometimes single ghost collisions. The latter are seen as the peak around 200 clusters per event and remain correctly untagged. The tail extending to a large number of clusters is consistent with the beam-gas simulation and is efficiently tagged as BIB. Finally, figure 20(d) shows that the BIB tagging efficiency is above $95 \%$ if there are $\gtrsim 500$ BIB pixel clusters in the event.

\subsection{BIB characteristics seen in 2011 data}

The pixel BIB tagging algorithm, described above, is applied to 2011 data to investigate the distribution of the BIB clusters in the Pixel detector and to assess the rate of BIB events as a function of the vacuum pressure upstream of the ATLAS detector.

The cluster $\phi$ distribution for each barrel layer is plotted in figure 21 for events which are selected by the algorithm as containing BIB. The distribution is normalised by the number of clusters in collision events, which are not selected by the algorithm, to reduce the geometrical effects of module overlaps and of the few pixel modules that were inoperable during this data-taking period. 


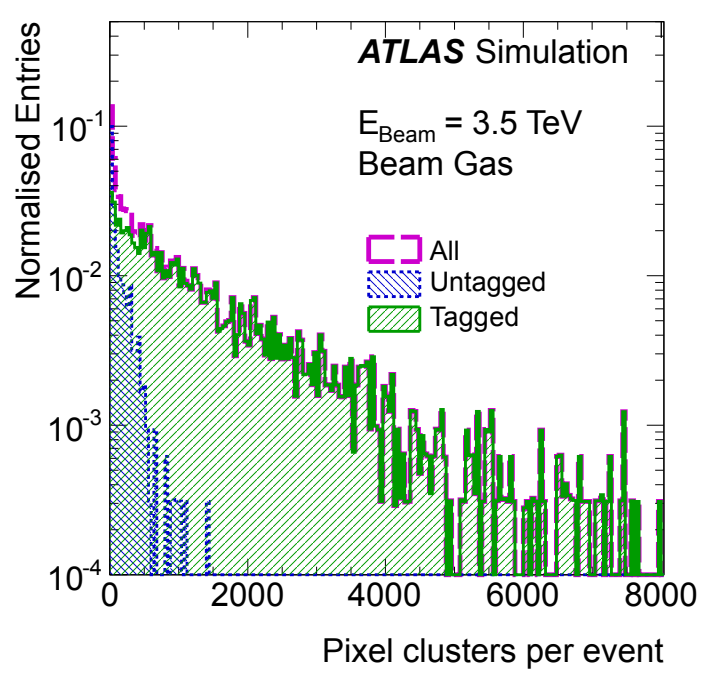

(a)

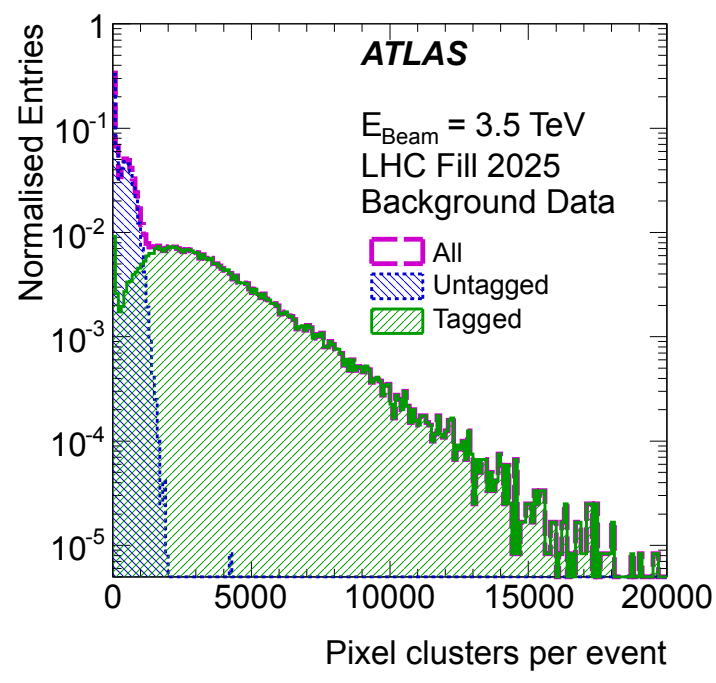

(c)

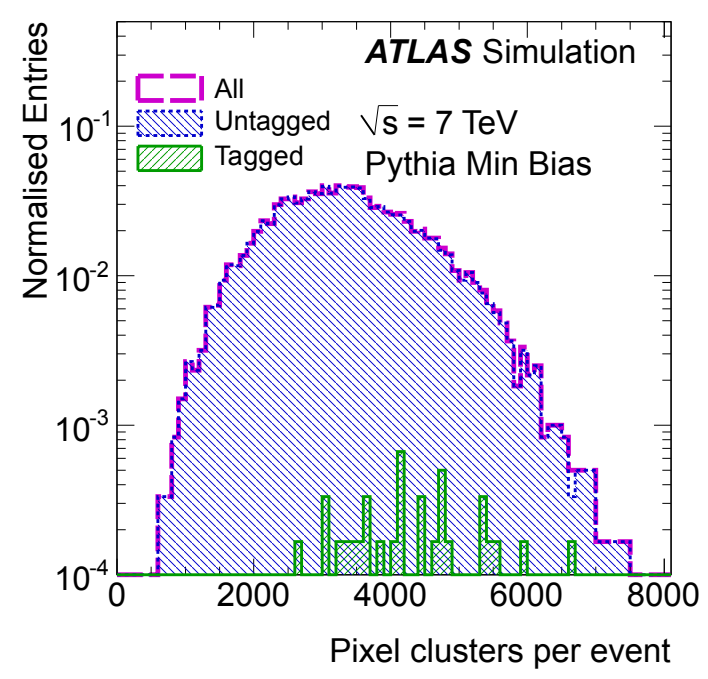

(b)

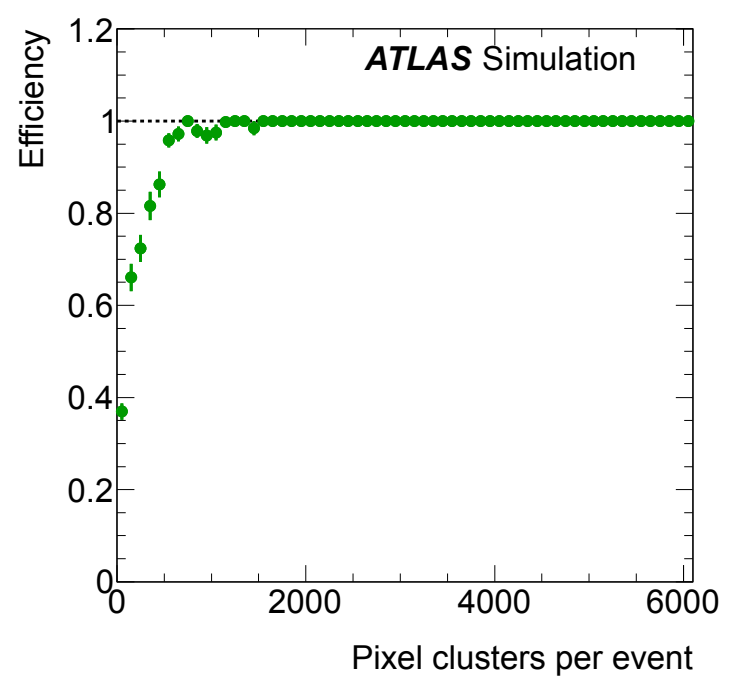

(d)

Figure 20. The pixel BIB tagging algorithm applied to: Monte Carlo samples of (a) beam-gas events, (b) minimum-bias collisions with pile-up of 21 events per bunch crossing, and (c) 2011 background data. The tagging efficiency as a function of the pixel cluster multiplicity is shown in (d). The efficiency is evaluated from the beam-gas simulation (a).

A small excess is observed at $\phi=0$ and $\phi=\pi$, corresponding to a horizontal spread of the BIB, most likely due to bending in the recombination dipoles. An up-down asymmetry is also apparent, which might be an artifact of the vertical crossing angle of the beams. Additional simulation studies are required to verify this hypothesis or to identify some other cause for the effect. ${ }^{12}$

\footnotetext{
${ }^{12}$ Since the Pixel detector is very close to the beam-line, the tunnel floor causing a similar effect in figure 7 cannot be the cause here.
} 


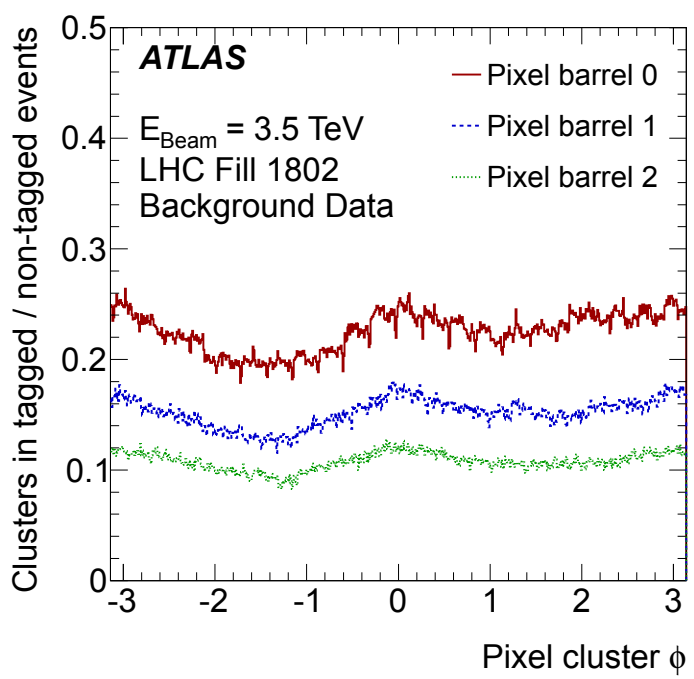

Figure 21. Azimuthal distribution of background tagged Pixel clusters, normalised by the cluster distribution in collision events.

\section{BIB muon rejection tools}

The BIB muon rejection tools described in this section are based on timing and angular information from the endcap muon detectors and the barrel calorimeters, and are primarily designed to identify fake jets due to BIB. The events to which the rejection tool is applied are typically selected by jet or $E_{\mathrm{T}}^{\text {miss }}$ triggers.

\subsection{General characteristics}

At radial distances larger than those covered by the acceptance of the tracking detectors, BIB can be studied with the calorimeters and the muon system. The LAr barrel has a radial coverage from 1.5 to $2.0 \mathrm{~m}$ and is therefore entirely covered by the radial range of the Cathode-Strip Chambers (CSC). The TileCal covers the radial range of $2.2 \mathrm{~m}<r<4.3 \mathrm{~m}$ which fully overlaps with the acceptance of the inner endcaps of the Monitored Drift Tube (MDT) system.

The left plot in figure 22 compares the $\phi$ distribution of the leading jets in data from unpaired bunches and from collisions. Both samples have general data quality requirements applied. Furthermore, the unpaired bunches are cleaned from ghost collisions by removing events with a reconstructed primary vertex. A striking difference is observed between the azimuthal distribution of leading jets from collisions and BIB. Whereas for collisions there is no preferred $\phi$ direction of jets, the azimuthal distribution for fake jets from BIB has two peaks, at $\phi=0$ and $\phi=\pi$. The region between the two peaks is somewhat more populated for $\phi>0$ than for $\phi<0$. These features are also seen in figure 7 and are explained by the arrangement of the dipole magnets and the shielding effect of the tunnel floor, respectively. The right plot in figure 22 shows that the reconstructed time of the fake jets from BIB is typically earlier than for jets from collisions. Physics objects from collisions have time $t \sim 0$ ns since all the time measurements are corrected for the time-of-flight from the interaction point

$$
t_{\mathrm{ToF}}=\sqrt{r^{2}+z^{2}} / c
$$



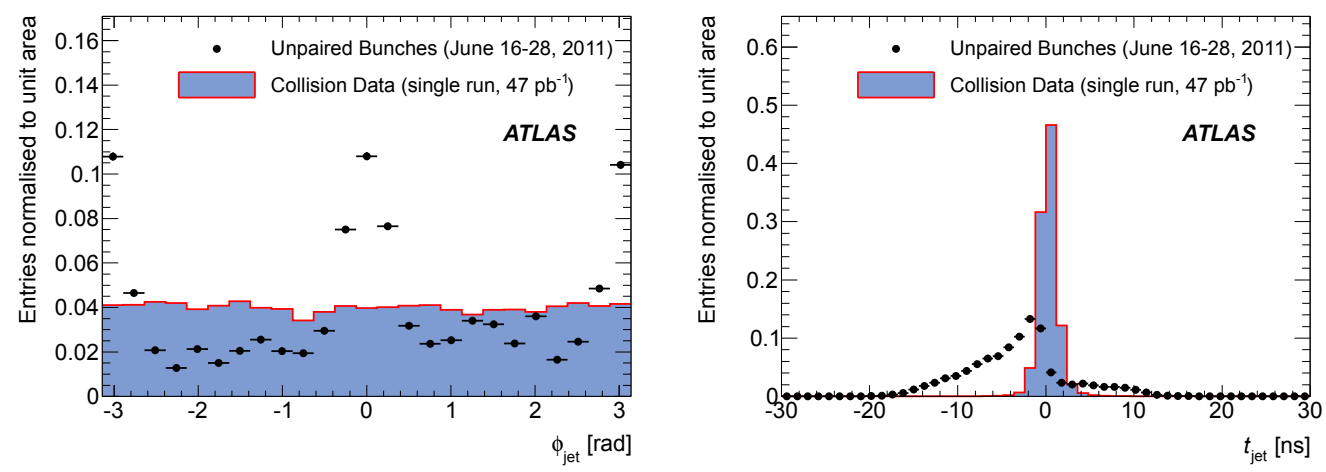

Figure 22. Leading jet $\phi$ (left) and time (right) in unpaired bunches and collision data.

where $(r, z)$ is the position of the physics object and $c$ is the speed of light. Since the highenergy components of BIB arrive simultaneously with the proton bunch, the BIB objects have time $t \sim \pm|z| / c$ with respect to the interaction time, where the sign depends on the direction of the BIB particle. As the reconstructed times are corrected for the time-of-flight, the reconstructed time of the BIB objects can be calculated as

$$
\begin{array}{ll}
t_{\mathrm{BIB}}=-z / c-t_{\mathrm{ToF}} & \text { for the } \mathrm{A} \rightarrow \mathrm{C} \text { direction, } \\
t_{\mathrm{BIB}}=+z / c-t_{\mathrm{ToF}} & \text { for the } \mathrm{C} \rightarrow \mathrm{A} \text { direction. }{ }^{13}
\end{array}
$$

These equations explain the observed time distribution in figure 22 as $t_{\mathrm{BIB}}$ is negative for the $z$ position where the BIB particle enters the detector and increases towards $0 \mathrm{~ns}$ on its way out of the detector on the other side. The entries at $t_{\text {jet }}>0 \mathrm{~ns}$ in the unpaired-bunch data are due to pile-up from the neighbouring interleaved bunches that are separated by only a $25 \mathrm{~ns}$ bunch spacing.

The response of the muon chambers to energetic BIB muons differs from that to muons from collisions, primarily due to their trajectories but also due to the early arrival time of the BIB muons with respect to the collision products. Figure 23 shows sketches of both of these characteristic features of BIB compared to the collision particles. The BIB particles have direction nearly parallel to the beam-pipe, therefore $\theta_{\mathrm{pos}}-\theta_{\mathrm{dir}} \sim \theta_{\mathrm{pos}}$, where $\theta_{\mathrm{pos}}, \theta_{\mathrm{dir}}$ denote the reconstructed polar position and direction, respectively. The collision products point to the interaction point and hence have $\theta_{\text {pos }}-\theta_{\text {dir }} \sim 0$. The reconstructed time of the BIB particles follows from eqs. (7.2) and (7.3). For the endcap chambers, the BIB particles can arrive either in time or early and the expected time can be formulated as

$$
\begin{aligned}
t_{\text {in-time }} & =+|z| / c-t_{\mathrm{ToF}}, \\
t_{\text {early }} & =-|z| / c-t_{\mathrm{ToF}} .
\end{aligned}
$$

For $z \gg r$, the time-of-flight correction in eq. (7.1) simplifies to $t_{\mathrm{ToF}} \sim|z| / c$. As the reconstructed times are corrected for the time-of-flight, the time of the BIB particles is either $t \sim(+|z|-|z|) / c=0$ or $t \sim(-|z|-|z|) / c=-2|z| / c$, depending on where along the path of the BIB particle through the detector the object is reconstructed. This approximation is illustrated in figure 23(b).

\footnotetext{
${ }^{13}$ The $z$-axis in the ATLAS coordinate system points from C to A.
} 


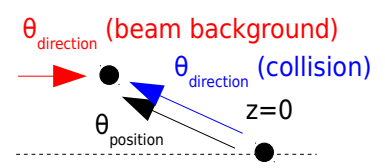

(a)

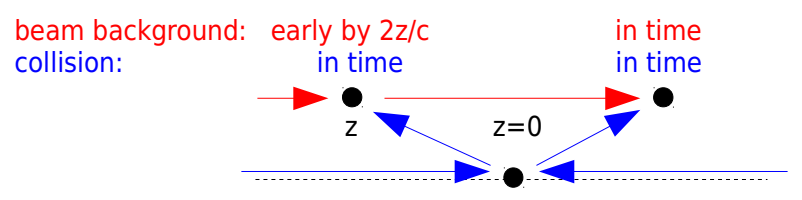

(b)

Figure 23. (a) Polar position and direction and (b) reconstructed time of the BIB objects compared to the collision objects.
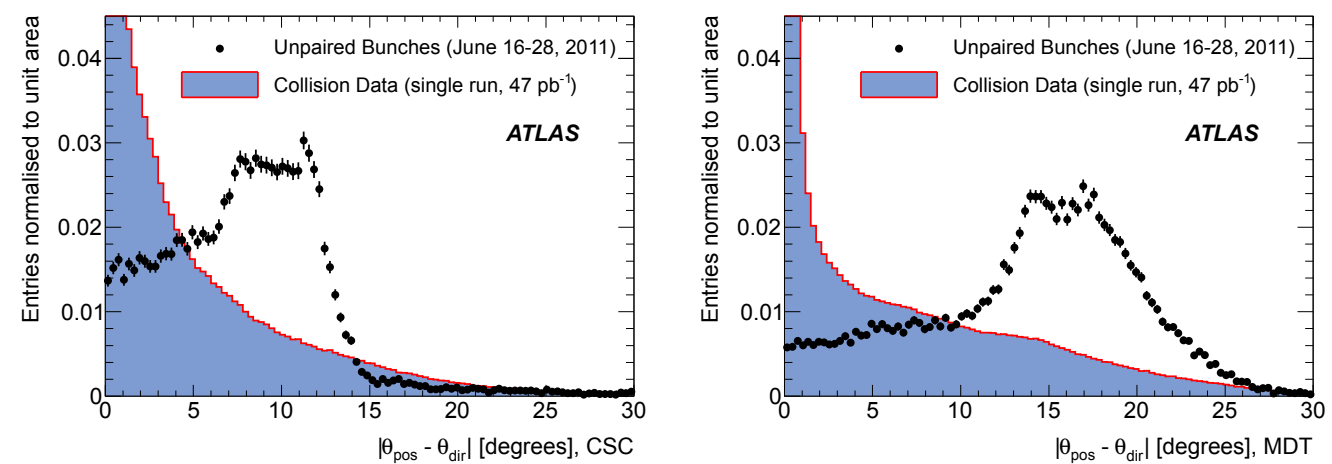

Figure 24. Difference between the reconstructed polar position $\theta_{\text {pos }}$ and the reconstructed polar direction $\theta_{\text {dir }}$ for the muon segments in the CSC (left) and the inner MDT endcap (right). Data from cleaned unpaired bunches (points) are compared to collisions (filled histogram).

Hits in each muon station are grouped into segments which allow the reconstruction of the direction of the particle causing the hits. At least three hits are required in order to form a segment. Figure 24 shows the difference between the reconstructed polar position $\theta_{\text {pos }}$ and the reconstructed polar direction $\theta_{\text {dir }}$ of the muon segments in the CSC and the inner MDT endcaps in cleaned unpaired bunches and collision data which, as can be seen in figure 23(a), is expected to be $\sim 0$ in collisions. This is indeed seen in figure 24 where the entries for collisions at non-zero values are due to angular resolution and particles bending in the toroidal magnetic field. For BIB, where $\Delta \theta=\left|\theta_{\text {pos }}-\theta_{\text {dir }}\right| \sim \theta_{\text {pos }}$, the expected values are $7^{\circ}<\Delta \theta<14^{\circ}$ for the CSC and $\Delta \theta>14^{\circ}$ for the inner MDT endcaps. The data clearly support the hypothesis that BIB muons are traversing the detector parallel to the beam-line at radii beyond $1 \mathrm{~m}$.

Figure 25 shows the transverse position of the muon segments that have direction nearly parallel to the beam-pipe in the CSC and the inner MDT endcaps. This is assured by requiring $\Delta \theta>5^{\circ}$ for the CSC and $\Delta \theta>10^{\circ}$ for the inner MDT endcaps. Only data from unpaired bunches are used in this plot, and the requirement on the direction of the muon segments helps to reject contributions coming from ghost collisions and noise. Such muon segments are referred to as "BIB muon segments" in the text below. It is seen that the charged BIB particles are mostly in the plane of the LHC ring $(y=0)$. Most of the muon segments are located at $r \sim 1.8 \mathrm{~m}$ and the distribution is steeply falling further away from the beam-pipe. The radial dependence and $\phi$-asymmetry are qualitatively consistent with figures 6 and 7, respectively. However, for BIB to be seen in data, the events have to be triggered. This is mostly done by jet triggers, which require calorimeter activity. The inner edge of the LAr barrel is at $r=1.5 \mathrm{~m}$ which explains why the rise of BIB rates 


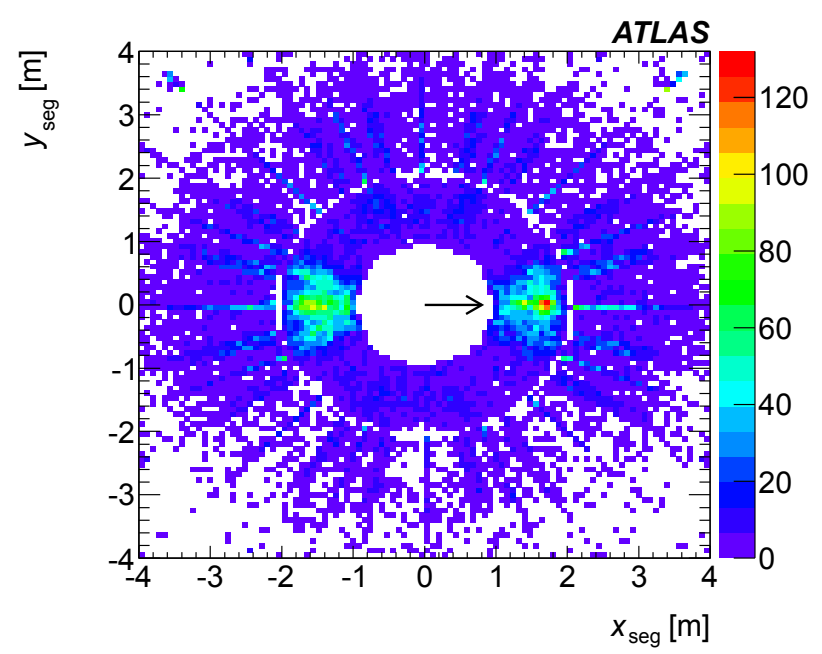

Figure 25. Position of the muon segments in the CSC and the inner MDT endcaps with a direction nearly parallel to the beam-pipe in the cleaned unpaired bunches. The arrow indicates the direction towards the centre of the LHC ring (positive $x$-axis). Units correspond to the number of entries per bin.

towards smaller radii, seen in figure 6 , is not reflected in the data. The jet triggers predominantly select highly energetic BIB muons that penetrate into the calorimeters and leave significant energy depositions above the trigger $p_{\mathrm{T}}$ threshold. Therefore, the pronounced azimuthal asymmetry of the muon segments observed in figure 25 corresponds mainly to high-energy BIB and fully reflects the jet asymmetry seen in figure 22 .

Figure 26 shows the reconstructed time of the BIB muon segments in cleaned unpaired bunches and collision data. As stated above, the collision products arrive at $t \sim 0 \mathrm{~ns}$. As expected, the time distribution of the muon segments in the inner MDT endcaps from collision data shows only a peak centred around $0 \mathrm{~ns}$. However, for the CSC muon segments there are two extra peaks in the time distribution located at $\pm 50 \mathrm{~ns}$. These peaks are related to the out-of-time pile-up due to the $50 \mathrm{~ns}$ bunch spacing. No such peaks are visible for the MDT endcaps since the reconstruction algorithm for the MDT is written in such a way that the out-of-time objects are suppressed. Furthermore, it can be seen that the whole time distribution for the CSC is shifted by $6.25 \mathrm{~ns}$ to positive values. ${ }^{14}$ In unpaired bunches, muon segments are expected to be either in time ( $\left.t \sim 0 \mathrm{~ns}\right)$ or early ( $t \sim-50 \mathrm{~ns})$ depending on whether the muon segment is created while exiting or entering the detector (see figure $23(\mathrm{~b})$ ). The expected time of $\sim-50 \mathrm{~ns}$ corresponds to the time-of-flight between the muon stations on both sides of the detector that are located at $|z| \sim 8 \mathrm{~m}$, and also coincides with the time of the early out-of-time pile-up.

As discussed in section 5.4, in some of the 2011 LHC bunch patterns, interleaved unpaired bunches were created by shifting the bunch trains to overlap with each other. In these cases bunches in opposite directions were separated by only $25 \mathrm{~ns}$. The peaks at $\pm 25 \mathrm{~ns}$ which are visible in the unpaired bunches in figure 26 correspond to muon segments reconstructed from the neighbouring

\footnotetext{
${ }^{14}$ This is due to the fact that half of the CSC channels have a $12.5 \mathrm{~ns}$ shift that is not corrected. Therefore, depending on which CSC channels are used for the time reconstruction, the muon segment time is shifted by $0,6.25$ or $12.5 \mathrm{~ns}$. The three distinct peaks are not visible in the distribution due to insufficient time resolution.
} 

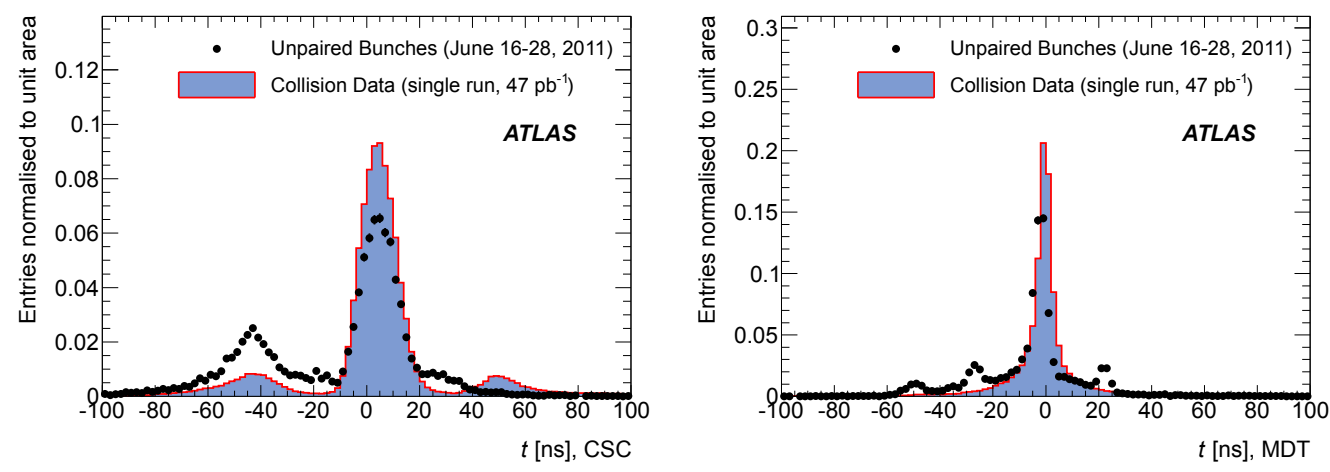

Figure 26. Reconstructed time of the CSC muon segments (left) and the inner MDT endcap segments (right) with a direction nearly parallel to the beam-pipe. Data from cleaned unpaired bunches (points) are compared to collisions (filled histogram).

interleaved unpaired bunch. The amount of data entering these peaks is about $10 \%$ of all unpairedbunch data.

A muon that radiates enough energy to create a fake jet loses a significant fraction of its energy, which is associated with a non-negligible momentum transfer. If the deflection, to which the endcap toroid field might also contribute in the case of MDT segments, is large enough, the outgoing muon would not create a muon segment with $\theta_{\text {dir }} \sim 0$ on the other side of the detector, or it might even miss the CSC or the inner MDT endcap altogether. Therefore, the number of entries in the early peak is expected to be larger than in the in-time peak. The fact that fewer early muon segments are seen is due to the muon segment reconstruction that is optimised for in-time measurements. Some of the early CSC segments are lost due to the fact that the read-out time window is not wide enough to detect all the early hits. As for the MDT segments, the out-of-time objects are suppressed by the reconstruction algorithm.

\subsection{BIB identification methods}

The characteristic signatures of BIB described above motivate a set of BIB identification methods. These either utilise only the basic information (position, direction, time) of the muon segments, or they try to match the muon segments to the calorimeter activity.

\subsubsection{Segment method}

The segment method requires the presence of a BIB muon segment, where $\theta_{\mathrm{dir}} \sim 0$, in the CSC or the inner MDT endcap. This method is very efficient for cleaning the empty bunch-crossings from BIB. Since the method is completely independent of calorimeter information, it is suitable for creating background-free empty bunch samples needed to identify noisy calorimeter cells.

\subsubsection{One-sided method}

The one-sided method requires the BIB muon segments and calorimeter clusters, with energy larger than $10 \mathrm{GeV}$, to be matched in relative azimuthal and radial positions. The matching in $\phi$ is motivated by the fact that BIB muons are not bent azimuthally by the magnetic fields of the ATLAS detector. The matching in $r$ is introduced in order to reduce the mis-identification probability of this method due to accidental matching. While the toroidal field does bend the trajectory in $r$, it 

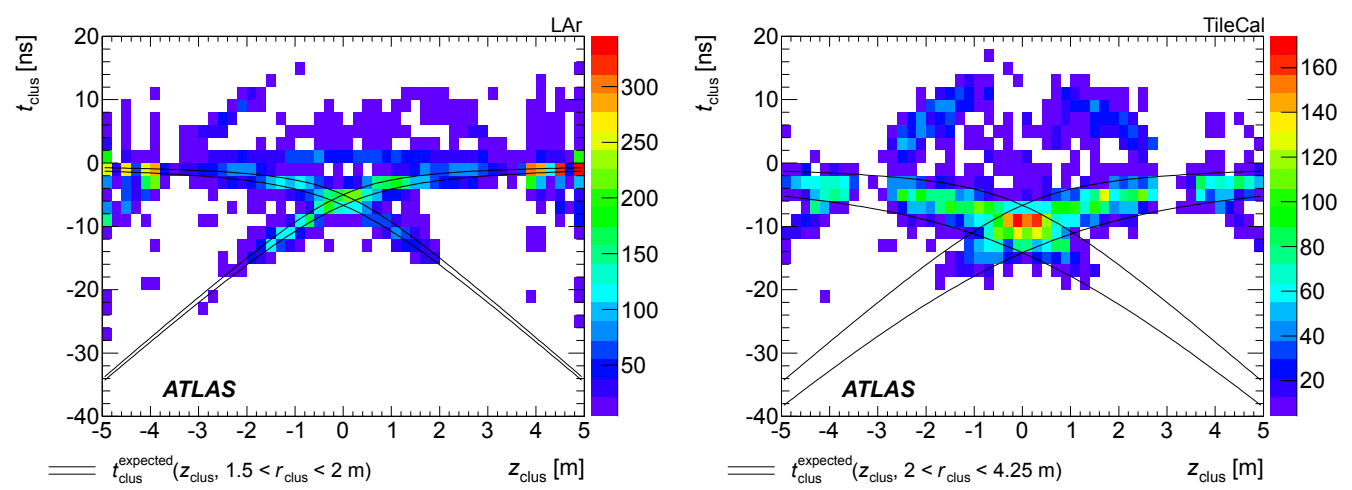

Figure 27. Cluster time plotted as a function of its $z$ position in the LAr (left) and TileCal (right) for the cleaned unpaired bunches. Only the clusters matching a BIB muon segment are shown. The two bands, covering the radial extent of the detectors, show the expected time for the BIB clusters in $1.5 \mathrm{~m}<r<2 \mathrm{~m}$ for $\mathrm{LAr}$ and $2 \mathrm{~m}<r<4.25 \mathrm{~m}$ for TileCal going in the $\mathrm{A} \rightarrow \mathrm{C}$ or $\mathrm{C} \rightarrow \mathrm{A}$ direction. Units correspond to the number of entries per bin.

can be assumed that the radial deflection remains small for high-energy incoming muons, or muons at radii below the inner edge of the endcap toroid. Ignoring the low-energy clusters also helps to suppress accidental matching. Depending on whether the muon segment is early or in time and on its position, the direction of the BIB muon may be reconstructed. The early (in-time) muon segments are selected such that the difference between the reconstructed time and the expected time $t_{\text {early }}\left(t_{\text {in-time }}\right)$, defined in eqs. (7.4) and (7.5), is less than $25 \mathrm{ns,} \mathrm{where} \mathrm{the} \mathrm{value} \mathrm{is} \mathrm{conservatively}$ chosen as half of the time-of-flight difference between the muon chambers on side A and side $\mathrm{C}$. The position of the calorimeter cluster in $z$ and $r$ can be used to estimate the expected time of the calorimeter energy deposition according to eqs. (7.2) or (7.3). Since the time resolution of the calorimeter measurements is $\sim 1 \mathrm{~ns}$ one can precisely compare the reconstructed cluster time with the expected value. The difference is required to be less than $2.5 \mathrm{~ns}$ in order to flag the cluster as a BIB candidate.

Figure 27 shows the cluster time as a function of the cluster $z$-position in unpaired bunches separately for the LAr and the TileCal. Expected cluster times for the radial acceptance of the calorimeters based on eqs. (7.2) and (7.3) are also indicated for both directions of BIB. The majority of data is seen to fall within the expectation band. However, there are also other interesting features in the plot: for the LAr calorimeter, there is a visible set of clusters with $t=0 \mathrm{~ns}$ at all $z$ positions. These come from the ghost collisions in the unpaired bunches. In both plots, one can see a set of clusters in a pattern similar to the expectation bands but shifted by $\sim 25 \mathrm{~ns}$ in time to positive values. These entries correspond to the clusters reconstructed from the neighbouring interleaved bunches, discussed already in section 7.1.

It follows from eqs. (7.2) and (7.3) that the expected time for BIB calorimeter clusters is close to $0 \mathrm{~ns}$ for small $r$ and large $|z|$ on the side where BIB leaves the detector. Therefore, the one-sided method has large mis-identification probability in the forward region.

Figure 28 shows the leading jet time as a function of its pseudorapidity in events identified by the one-sided method. It can be seen that the characteristic timing pattern of the BIB calorimeter clusters shown in figure 27 is reflected in the properties of the reconstructed jets due to BIB. 


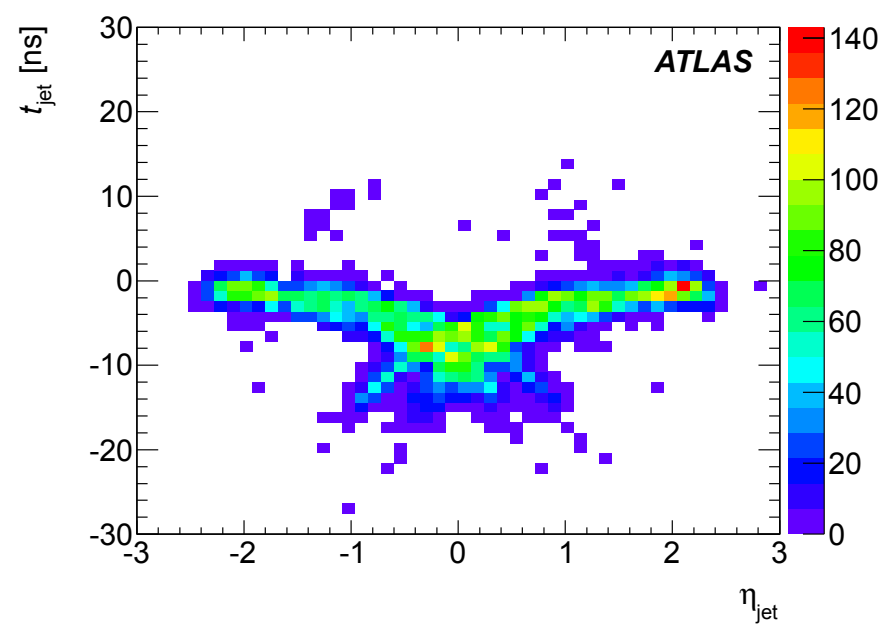

Figure 28. Time of the leading jet as a function of its $\eta$ in the cleaned unpaired bunches. Only the events identified by the one-sided method are shown.

\subsubsection{Two-sided method}

The two-sided method requires a BIB muon segment on both sides to be matched in $\phi$ and $r$ to a single calorimeter cluster of energy above $10 \mathrm{GeV}$. Here, the cluster time is not checked. A corresponding time difference between the two segments is required instead. The expected time difference, due to the relative $z$-position of the muon chambers on both sides of the spectrometer, is $\Delta t=50 \mathrm{~ns}$. Since the time resolution of the CSC is about $7 \mathrm{~ns}$ (see figure 26) a conservative cut of $\Delta t>25 \mathrm{~ns}$ is applied.

Such an event topology is unlikely to be mimicked by collision products which makes this method particularly robust against mis-identification.

\subsubsection{Efficiency and mis-identification probability}

The efficiency $(\varepsilon)$ of the identification methods is evaluated from the whole 2011 unpaired-bunch data. General data quality assessments are imposed on the sample, and ghost collisions are suppressed by vetoing events with one or more reconstructed primary vertices. Noisy events are further reduced by requiring a leading jet with a large transverse momentum of $p_{\mathrm{T}}>120 \mathrm{GeV}$. Jets from the inner part of the calorimeter endcaps, where there is no overlap with any muon chamber, are suppressed by rejecting events with the leading jet $|\eta|>2.8$. However, the number of events with the leading jet outside the calorimeter barrel, $|\eta|>1.5$, is negligible anyway.

The mis-identification probability $\left(P_{\text {mis }}\right)$ is determined in a back-to-back dijet sample from collision data. This sample also meets the general data quality requirements and the events with at least two jets as well as leading jet transverse momentum $p_{\mathrm{T}}>120 \mathrm{GeV}$ and $|\eta|<2$ are selected. Furthermore, the second leading jet in this sample is required to have a similar transverse momentum to the first one $\left(\frac{\left|p_{\mathrm{T}}^{1}-p_{\mathrm{T}}^{2}\right|}{p_{\mathrm{T}}^{1}+p_{\mathrm{T}}^{2}}<0.2\right)$ and the two jets are required to be back-to-back in the transverse plane $\left(\Delta \phi_{\mathrm{j}-\mathrm{j}}>2.8\right)$. An event is mis-tagged as BIB if any of the muon segments or calorimeter clusters satisfy the requirements of the tagging methods discussed above. 


\begin{tabular}{|l|l|l|}
\hline method & efficiency \pm (stat) & mis-identification probability \\
\hline segment & $0.816 \pm 0.017$ & 0.46 \\
one-sided & $0.542 \pm 0.013$ & 0.014 \\
two-sided & $0.160 \pm 0.006$ & $10^{-5}$ \\
\hline
\end{tabular}

Table 2. Efficiency and mis-identification probability of the BIB identification methods. The misidentification probabilities are derived from high-statistics samples, therefore no statistical uncertainties are given.

The resulting $\varepsilon$ and $P_{\text {mis }}$ are listed in table 2. The high efficiency of the segment method (81.6\%) makes it useful in preparing background-free samples or for data quality monitoring. In physics analyses however, it is important to clean background with a minimum loss of signal events. Table 2 shows that the two-sided method has high purity, $P_{\text {mis }}=10^{-5}$, but has an efficiency of only $16.0 \%$. The one-sided method has a better efficiency of $54.2 \%$, but $\sim 1.4 \%$ of signal events are mis-identified. However, the numbers given for the mis-identification probabilities also depend on the final-state topology induced by the signal region cuts in a particular physics analysis. Therefore, the mis-identification probabilities given here serve only as an illustration where dijets are chosen as an example. The combined efficiency of the one-sided and the two-sided methods yields $56.7 \%$ for the OR combination and $13.5 \%$ for the AND combination.

It was shown previously that the interleaved bunches may cause BIB from one BCID to be reconstructed in a neighbouring BCID with a reconstructed time shifted accordingly by $25 \mathrm{~ns}$. This introduces a systematic bias to the evaluated efficiencies of the one-sided and two-sided methods since they select BIB predominantly from the current BCID. The fraction of BIB, reconstructed from the neighbouring interleaved bunches, in all unpaired-bunch data is approximately $10 \%$ and it is not certain to what extent there is double counting of such events in the sample. Therefore, $10 \%$ is also taken as a relative systematic uncertainty.

\subsection{BIB rate in 2011}

The two-sided method is used to evaluate the rate of BIB in the whole 2011 collision data set. Figure 29(a) shows the time evolution of the BIB rate normalised to the nominal bunch current of $10^{11}$ protons. The plot shows that the rate was high early in the year and then after the first technical stop (TS1) rather rapidly decreased by a factor $\sim 3$, staying at a fairly constant level after early June. The only exceptions are the first runs after technical stops 2 and 3, where higher rates are observed.

In unpaired bunches, the rate is evaluated using the AND combination of the two-sided and onesided methods. The former one is chosen in order to maintain the low mis-identification probability. The latter one helps to remove the BIB reconstructed from the neighbouring interleaved bunches. ${ }^{15}$ Figure 29(b) shows the BIB rate in unpaired isolated and unpaired non-isolated bunches. As in the filled bunches, higher rates before May and just after the technical stops are also visible here. The rates in filled and unpaired bunches cannot be compared quantitatively since different triggers were used and no trigger efficiency corrections are applied here.

\footnotetext{
${ }^{15}$ Removing the entries from the neighbouring interleaved bunches is important in particular for evaluating rates for beam-1 and beam-2 separately.
} 


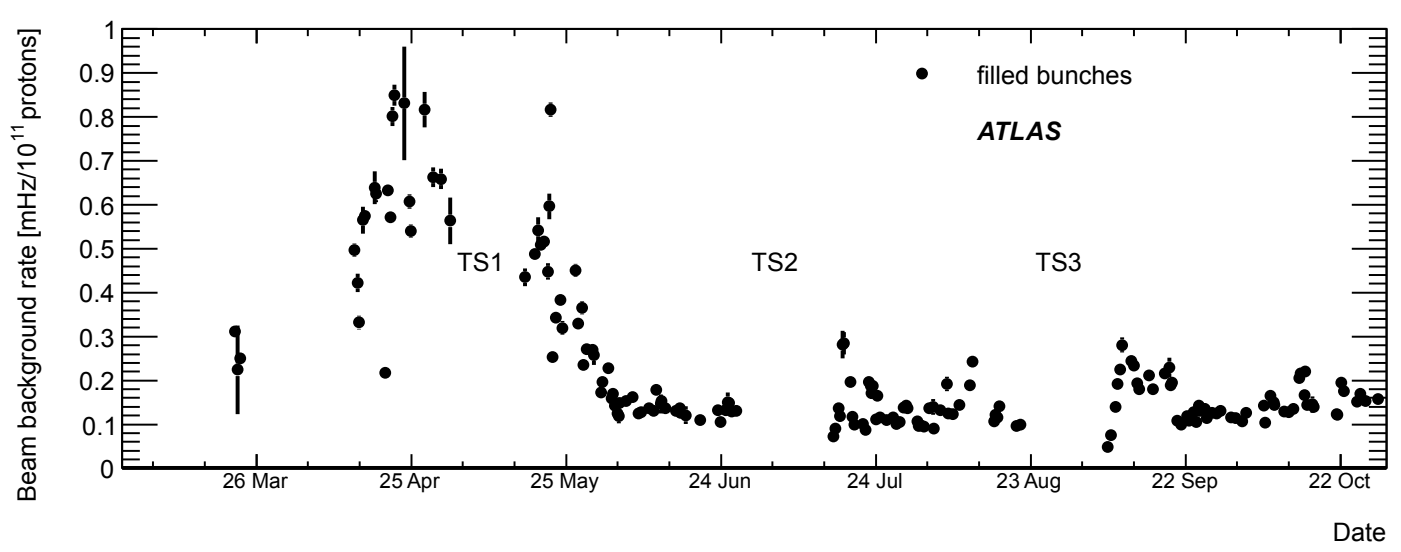

(a) BIB rate in filled bunches.

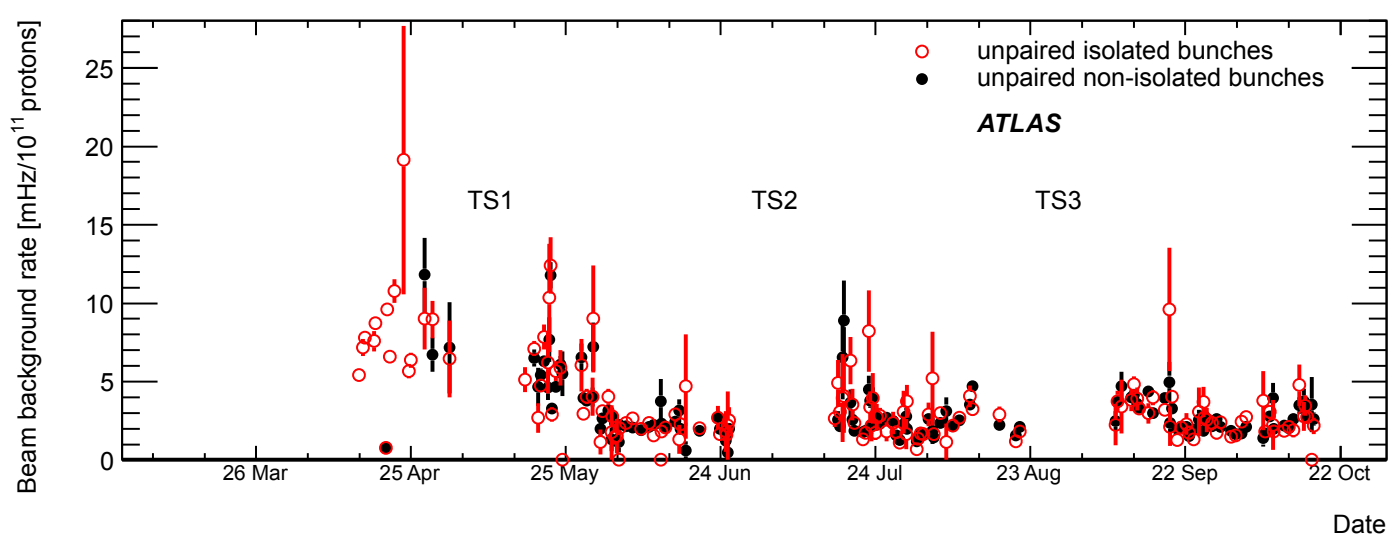

(b) BIB rate in unpaired isolated and unpaired non-isolated bunches.

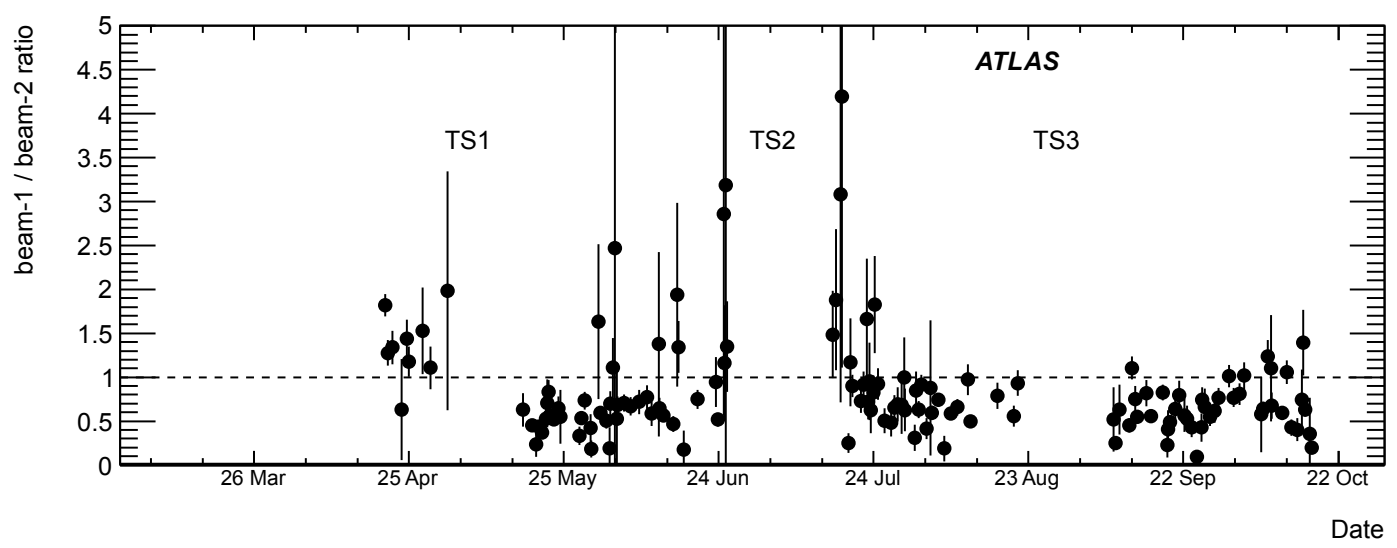

(c) Ratio of the BIB rate in beam-1 and beam-2 in the unpaired bunches.

Figure 29. BIB rate in 2011 proton-proton runs. The rates in filled (a) and unpaired (b) bunches cannot be compared quantitatively because of different trigger requirements. One entry in the plot corresponds to one LHC fill. Only the statistical uncertainties are shown. Technical stops are indicated in the plot. 
The data from unpaired isolated and unpaired non-isolated bunches are two statistically independent samples and the corresponding rates should be in agreement. The ratio of the measured rates for all data after the first technical stop is $0.974 \pm 0.018$ where only the statistical uncertainties are considered. Possible explanations for the relative difference are dead time and different trigger efficiency depending on the relative position of unpaired bunches with respect to the colliding bunch-trains.

The identification methods also enable the direction of BIB muons to be reconstructed. This allows the determination of whether the BIB muon originates in beam-1 or beam-2. Figure 29(c) shows the comparison of the BIB rate for beam- 1 and beam- 2 separately using the data from unpaired bunches. Averaged over the entire year the rate in beam- 1 is lower than in beam- 2 by a factor of $0.57 \pm 0.01$, taking only the statistical uncertainty into account. But it is also evident from figure 29(c) that the ratio differs from fill to fill and the origin of the asymmetry has not yet been identified. As discussed in the context of LHC collimation, there is no reason to believe that the beam halo should be equal for both beams. Attempts were made to correlate the relative rates with beam losses in the cleaning insertions, but no clear correlations could be found. Most likely other BIB sources, such as variations in vacuum quality, which can be different for the two beams to some extent, also play a role.

\section{Removal of non-collision background with jet observables}

The term "non-collision backgrounds" refers to the sources of backgrounds that are not related to the proton-proton collision products. These comprise BIB, cosmic rays and noise. This section describes a method to remove non-collision background in physics analyses based on jet observables, with a special emphasis on BIB. A set of jet cleaning cuts, which are commonly used in ATLAS analyses, is introduced first. It is then shown how non-collision backgrounds can be further reduced and how to estimate their residual levels. Finally, an example of the monojet signatures search [34] illustrates the performance of the standard cleaning techniques.

\subsection{Jet cleaning}

The jet selection criteria should effectively reject jets due to background processes while keeping high efficiency for jets produced in proton-proton collisions. Since the level and composition of the background depends on the event topology and the jet kinematics, several criteria are proposed, corresponding to different levels of fake jet rejection and jet selection efficiency.

\subsubsection{Event samples}

The selection criteria, based on jet quality, are optimised by studying event samples enriched in jets from collisions or in fake jets. Events are classified mainly by the missing transverse momentum significance, defined as $E_{\mathrm{T}}^{\text {miss }} / \sqrt{\Sigma E_{\mathrm{T}}}$, where $E_{\mathrm{T}}^{\text {miss }}$ is the missing transverse momentum [35] and $\Sigma E_{\mathrm{T}}$ is the scalar sum of the transverse energies of all energy deposits in the calorimeter.

- The collision jet sample requires two jets with $p_{\mathrm{T}}^{\text {jet }}>20 \mathrm{GeV}$ that are back-to-back in the transverse plane $\left(\Delta \phi_{\mathrm{j}-\mathrm{j}}>2.8\right)$ and have small missing transverse momentum significance $E_{\mathrm{T}}^{\text {miss }} / \sqrt{\Sigma E_{\mathrm{T}}}<2 \mathrm{GeV}^{1 / 2}$. Events are selected by single-jet triggers [36], where the threshold 
is chosen such that the trigger is fully efficient $(>99 \%)$ in the considered $p_{\mathrm{T}}^{\text {jet }}$-bin. The selected sample is dominated by dijet events and is called "sample enriched in collision jets" in the following.

- Fake jets are selected from events with only one jet with $p_{\mathrm{T}}^{\text {jet }}>150 \mathrm{GeV}$, large missing transverse momentum $E_{\mathrm{T}}^{\text {miss }}>150 \mathrm{GeV}$ and large missing transverse momentum significance $E_{\mathrm{T}}^{\text {miss }} / \sqrt{\Sigma E_{\mathrm{T}}}>3 \mathrm{GeV}^{1 / 2}$. The transverse component of the jet momentum is required to be opposite to the missing transverse momentum direction $\left(\Delta \phi_{E_{\mathrm{T}}^{\text {miss }}-\mathrm{j}}>2.8\right)$. Events with sub-leading jets with $p_{\mathrm{T}}^{\text {jet }}>40 \mathrm{GeV}$ or with reconstructed leptons are discarded. The events are triggered by requiring the presence of a jet and missing transverse momentum. The trigger thresholds are chosen to be fully efficient with respect to the selection criteria described above. This event sample is dominated by BIB, with a negligible contamination from calorimeter noise and physics processes like $Z \rightarrow v v+$ jets and $W \rightarrow \ell v+$ jets. In the following, this event sample is called "sample enriched in fake jets".

For both samples, requirements that ensure the quality of beam conditions, detector performance and data processing are imposed. After application of these criteria, the total integrated luminosity is about $4.7 \mathrm{fb}^{-1}$.

\subsubsection{Criteria to remove non-collision background}

Beam-induced background and cosmic rays. The distribution of energy deposits by the jet, the shower shape and its direction, in particular the pointing to the interaction point, can be employed to discriminate collision jets from BIB-induced fake jets. Examples of discriminating variables are the electromagnetic energy fraction $\left(f_{\mathrm{EM}}\right)$, defined as the energy deposited in the electromagnetic calorimeter, divided by the total jet energy, and $\left(f_{\max }\right)$, the maximum energy fraction in any single calorimeter layer.

The vast majority of collision jets contain charged hadrons that are reconstructed by the tracking system. In the tracker acceptance, $|\eta|<2.5$, the jet charged particle fraction $\left(f_{\text {ch }}\right)$ is defined as the ratio of the scalar sum of the $p_{\mathrm{T}}$ of the tracks associated with the jet divided by $p_{\mathrm{T}}^{\text {jet }}$. This is another powerful tool to discriminate collision jets from fake jets, which typically have no associated tracks. Finally, BIB and cosmic rays induce jet candidates that are usually not in-time with the collision products.

Noise in the calorimeters. Most of the noise is already identified and rejected by the data quality inspection performed shortly after data-taking, based on standardised quality criteria. A small fraction of calorimeter noise remains undetected and needs to be rejected by additional criteria, because it can lead to reconstruction of energy deposits not associated with particle interactions in the calorimeter. As explained in section 3, the characteristic pulse shape of real energy deposits in the calorimeter cells can be used to distinguish a true ionisation signal from noise. This leads to the definition of the quality variables $f_{\mathrm{HEC}},\langle Q\rangle, f_{\mathrm{Q}}^{\mathrm{LAr}}$ and $f_{\mathrm{Q}}^{\mathrm{HEC}}$, described in section 3 .

Jet quality selections. Four sets of jet quality criteria — "Looser", "Loose", "Medium" and "Tight" - are defined in order to reject fake jets in 2011 data. These correspond to different background rejection factors and jet selection efficiencies. The selection criteria using jet quality 
to identify and reject fake jets are listed in table 3. The Looser criteria are designed to provide a signal efficiency above $99.8 \%$ with a fake-jet rejection factor of about $50 \%$, while the Tight criteria are designed to provide a large fake-jet rejection factor with a signal inefficiency not larger than a few percent. The two other sets of cuts correspond to intermediate rejection factors and selection efficiencies.

Figure 30 shows jet distributions for the sample enriched in fake jets before and after applying the selection criteria listed in table 3. Distributions from the sample enriched in collision jets are also superimposed where applicable.

As shown before, the two peaks at $\phi=0$ and $\phi=\pi$ are characteristic of BIB and are effectively removed only by the Tight selection criteria. The good agreement between the sample enriched in fake jets after the Tight selection criteria and the sample enriched in collision jets shows that the fake-jet background contamination is very small once the Tight selection criteria are applied. After this cleaning, the sample enriched in fake jets is dominated by physics processes like $Z \rightarrow v v+$ jets and $W \rightarrow \ell v+$ jets.

An "out-of-time" sub-set of the sample enriched in fake jets is selected by requiring $5<$ $\left|t_{\text {jet }}\right|<10 \mathrm{~ns}$. Since this time cut is not used in the fake-jet sample selection, this sub-sample provides a fake-jet sample that can be used to compute an independent estimate of the fake-jet rejection. The timing cut helps to reduce significantly the collision jet contamination in the sample enriched in fake jets (see figure 30). The Looser criteria reject 37.8\% (68.6\%) of the out-of-time fake jets with $p_{\mathrm{T}}^{\text {jet }}>150 \mathrm{GeV}(500 \mathrm{GeV})$, while the Tight criteria reject more than $99.9 \%$ of the jets in the out-of-time sub-set of the sample enriched in fake jets. The results are summarised in table 4. The valid $\eta$ ranges for all cuts are indicated in table 3 . Only overall efficiencies, integrated over the whole $\eta$ range, are given here, although variations depending on $\eta$ have to be expected. The efficiency of the $f_{\text {ch }}$ and $f_{\mathrm{EM}}$ selection criteria for fake jets is expected to be degraded with increasing pile-up compared to the 2011 data studied here, because the characteristic peaks at $f_{\text {ch }}=0$ and $f_{\mathrm{EM}}=0$ and 1 become broader.

\subsubsection{Evaluation of the jet quality selection efficiency}

The efficiency of the jet selection criteria is measured using the "tag-and-probe" method. Collision dijet events are selected as described in section 8.1.1. The tagging jet $\left(p_{\mathrm{T}}^{\text {ref }}\right)$ is required to pass the Tight selection criteria, and to be back-to-back with the probe jet $\left(p_{\mathrm{T}}^{\text {probe }}\right)$. The probe-jet sample is used to measure the jet selection efficiency defined as the fraction of probe jets selected, as a function of $\eta$ and $p_{\mathrm{T}}^{\text {jet }}$ of the probe jets.

The efficiency for the selection of good jets using the Looser criteria is better than $99.8 \%$ over all $p_{\mathrm{T}}^{\text {jet }}$ and $\eta$ bins while a slightly lower efficiency is measured for the Loose criteria in particular at low $p_{\mathrm{T}}^{\text {jet }}$ and for $2.5<|\eta|<3$.6. The Medium and Tight selection criteria have lower jet selection efficiency due mainly to the cuts on the jet charged particle fraction. For jets with $p_{\mathrm{T}}^{\text {jet }}$ of about $25 \mathrm{GeV}$, the Medium and Tight criteria have inefficiencies of $4 \%$ and $15 \%$ respectively. For $p_{\mathrm{T}}^{\text {jet }}>50 \mathrm{GeV}$, the Medium and Tight selection criteria have efficiencies better than $99 \%$ and $98 \%$, respectively.

The event selection (using $\Delta \phi_{\mathrm{j}-\mathrm{j}}$ and $E_{\mathrm{T}}^{\text {miss }} / \sqrt{\Sigma E_{\mathrm{T}}}$ cuts) and the Tight selection of the tag jet are varied to study the systematic uncertainties. For the Loose and Looser criteria, the jet selection 


\begin{tabular}{|l|l|}
\hline & Looser \\
\hline BIB and cosmic rays & $\begin{array}{l}\left(f_{\text {max }}>0.99 \text { and }|\eta|<2\right) \\
\text { or }\left(f_{\mathrm{EM}}<0.05 \text { and } f_{\text {ch }}<0.05 \text { and }|\eta|<2\right) \\
\text { or }\left(f_{\mathrm{EM}}<0.05 \text { and }|\eta| \geq 2\right)\end{array}$ \\
\hline $\begin{array}{l}\left(f_{\mathrm{HEC}}>0.5 \text { and }\left|f_{\mathrm{Q}}^{\mathrm{HEC}}\right|>0.5 \text { and }\langle Q\rangle>0.8\right) \\
\text { or }\left|E_{\text {neg }}\right|>60 \mathrm{GeV} \\
\text { or }\left(f_{\mathrm{EM}}>0.95 \text { and } f_{\mathrm{Q}}^{\mathrm{LAr}}>0.8 \text { and }\langle Q\rangle>0.8\right. \\
\text { and }|\eta|<2.8)\end{array}$ \\
\hline Loose \\
\hline BIB and cosmic rays & $\begin{array}{l}\text { Looser or } \\
\left|t_{\text {jet }}\right|>25 \text { ns }\end{array}$ \\
\hline Calorimeter noise & $\begin{array}{l}\text { Looser or } \\
\left(f_{\mathrm{HEC}}>0.5 \text { and }\left|f_{\mathrm{Q}}^{\mathrm{HEC}}\right|>0.5\right) \\
\text { or }\left(f_{\mathrm{EM}}>0.95 \text { and } f_{\mathrm{Q}}^{\mathrm{LAr}}>0.8 \text { and }|\eta|<2.8\right)\end{array}$ \\
\hline Medium \\
\hline BIB and cosmic rays & $\begin{array}{l}\text { Loose or } \\
\left|t_{\text {jet }}\right|>10 \text { ns } \\
\text { or }\left(f_{\mathrm{EM}}<0.05 \text { and } f_{\text {ch }}<0.1 \text { and }|\eta|<2\right) \\
\text { or }\left(f_{\mathrm{EM}}>0.95 \text { and } f_{\text {ch }}<0.05 \text { and }|\eta|<2\right)\end{array}$ \\
\hline Loose or \\
$f_{\mathrm{HEC}}>1-\left|f_{\mathrm{Q}}^{\mathrm{HEC}}\right|$ \\
or $\left(f_{\mathrm{EM}}>0.9\right.$ and $f_{\mathrm{Q}}^{\mathrm{LAr}}>0.8$ and $\left.|\eta|<2.8\right)$
\end{tabular}

Table 3. Selection criteria used to identify fake jets. They are classified from the loosest to the tightest one: Looser, Loose, Medium and Tight selection criteria.

\begin{tabular}{|c|c|c|c|c|c|}
\hline & Total & Looser & Loose & Medium & Tight \\
\hline$p_{\mathrm{T}}^{\text {jet }}>150 \mathrm{GeV}$ & 124890 & $77675(37.81 \%)$ & $70226(43.76 \%)$ & $663(99.46 \%)$ & $38(99.99 \%)$ \\
$p_{\mathrm{T}}^{\text {jet }}>500 \mathrm{GeV}$ & 2140 & $671(68.64 \%)$ & $652(69.53 \%)$ & $10(99.53 \%)$ & $0(100 \%)$ \\
\hline
\end{tabular}

Table 4. Number of jet candidates in the out-of-time sub-set of the sample enriched in fake jets before and after applying the jet selection criteria. Numbers in parentheses are the fraction of jets identified as fake jets. 

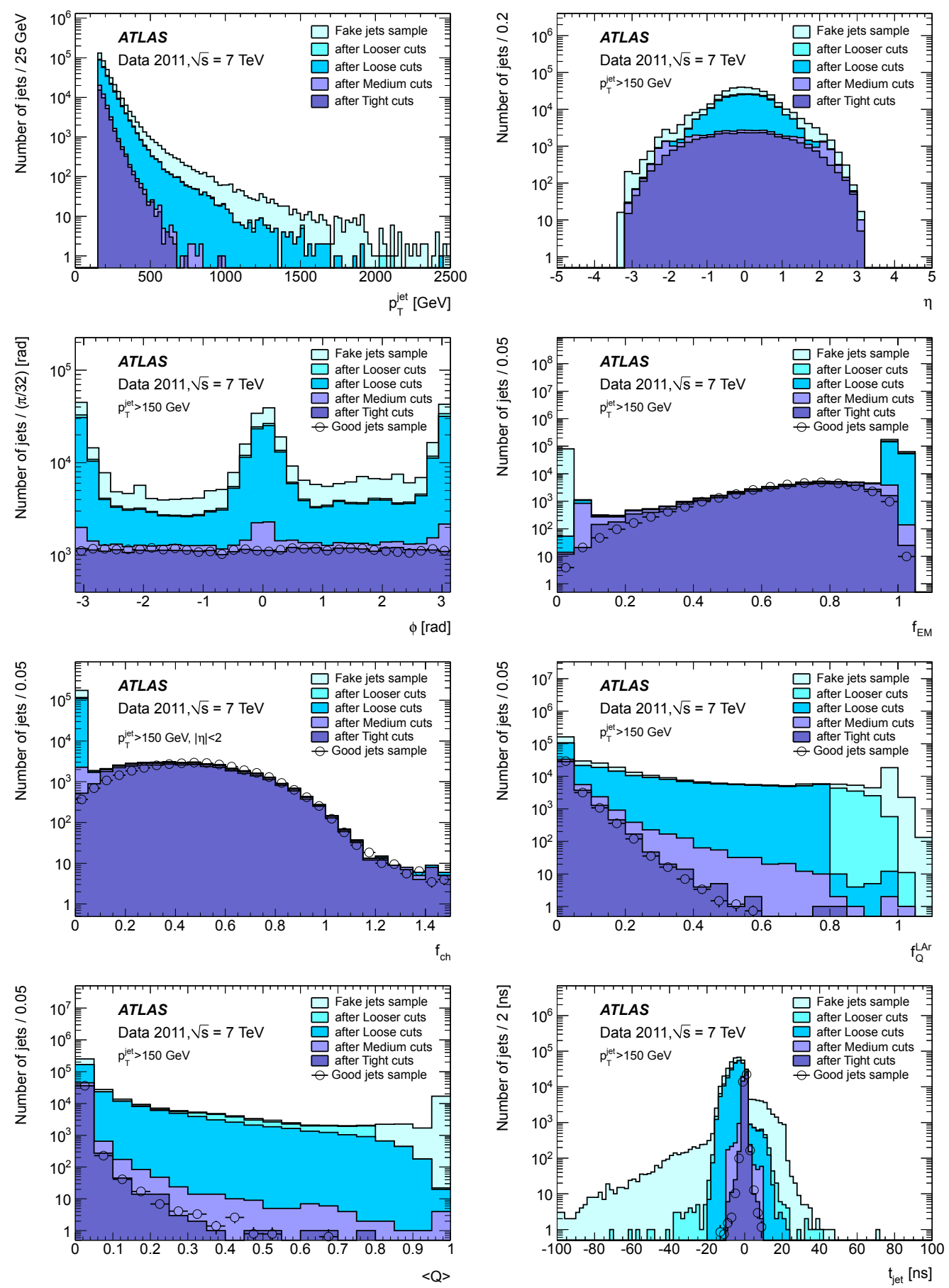

Figure 30. Distributions of jet kinematic and discriminating variables for the sample enriched in fake jets before and after applying the jet selection criteria. Distributions for the sample enriched in collision jets, labelled as "good jets sample" in the figures, are also superimposed where applicable. Distributions for jets from collisions are re-weighted in a way to reproduce the two-dimensional jet $p_{\mathrm{T}}^{\text {jet }}$ versus jet $\eta$ distribution obtained from the sample enriched in fake jets after Tight selection cuts. 
efficiency is almost unchanged (variations are smaller than $0.05 \%$ ) when varying the selection cuts. For the Medium (Tight) criteria the size of the variation is at most $0.1 \%(0.5 \%)$.

The jet selection efficiency is measured in multijet Monte Carlo samples and compared to the data driven estimates. Very good agreement is observed for the Looser and Loose criteria. For the Medium (Tight) selection criteria differences not larger than $0.2 \%(1 \%)$ are observed for $p_{\mathrm{T}}^{\text {jet }}>40 \mathrm{GeV}$. Differences at lower $p_{\mathrm{T}}^{\text {jet }}$ values are at most $1 \%(2 \%)$ for the Medium (Tight) selection criteria.

\subsection{Monojet analysis}

Events with a single jet balanced by large missing transverse momentum are often exploited to search for signatures of new physics. The monojet analysis searches for new exotic phenomena such as Supersymmetry, Large Extra Dimensions, an invisibly decaying Higgs boson or Dark Matter candidates. The analysis is carried out on data from proton-proton collisions at $\sqrt{s}=7 \mathrm{TeV}$ taken in 2011, corresponding to an integrated luminosity of $4.7 \mathrm{fb}^{-1}$. A detailed description of the analysis can be found in [34]. Only the BIB rejection methods are discussed here.

The dominant Standard Model physics processes that form an irreducible background in this analysis are $Z \rightarrow v v+$ jets, where a jet from initial-state radiation is detected and the two neutrinos create large $E_{\mathrm{T}}^{\mathrm{miss}}$, and $W \rightarrow \ell v+$ jets, where the lepton is out of the acceptance of the detector or badly reconstructed. Other backgrounds in the analysis, in decreasing order of importance, are top-quark decays, multijet production, non-collision backgrounds and diboson production $(W W$, $W Z, Z Z)$.

The events in the monojet analysis signal region are selected by $E_{\mathrm{T}}^{\text {miss }}$ triggers and must have a reconstructed primary vertex. Furthermore, events with reconstructed leptons are rejected. A leading jet with $p_{\mathrm{T}}^{\text {jet }}>120 \mathrm{GeV}$, accompanied by $E_{\mathrm{T}}^{\text {miss }}>120 \mathrm{GeV}$, is required. Events with a third jet with $p_{\mathrm{T}}^{\text {jet }}$ above $30 \mathrm{GeV}$ are vetoed. The veto on additional jets is less stringent than in the previous ATLAS monojet search [37] as it was shown that allowing a second jet in the event reduces systematic uncertainties from initial- or final-state radiation and increases signal selection efficiencies. If a second jet exists, the difference between the azimuthal angle of the second jet and $E_{\mathrm{T}}^{\mathrm{miss}}$ is required to be larger than $0.5 \mathrm{rad}$. This cut suppresses back-to-back QCD dijet events where one of the jets is mis-measured resulting in $E_{\mathrm{T}}^{\text {miss }}$ pointing in its direction. This set of cuts is referred to as the "monojet selection".

The monojet selection and the selection for the sample enriched in fake jets, defined in section 8.1.1, are remarkably similar. Indeed, it is shown below that the monojet selection tends to select predominantly non-collision background events and therefore the analysis requires efficient cleaning of BIB and cosmic rays.

While most ATLAS physics analyses require only the Looser jet selection criteria introduced in table 3, the monojet analysis requires the Medium criteria. This applies to all jets with $p_{\mathrm{T}}^{\text {jet }}>20 \mathrm{GeV}$ in an event. An additional cut on the leading jet charged particle fraction $f_{\text {ch }}>0.02$ and electromagnetic energy fraction $f_{\mathrm{EM}}>0.1$ is imposed in order to obtain even higher rejection power.

Figure 31 illustrates the importance of the cleaning cuts in the monojet analysis. The leading jet $\phi$ distribution, before applying any cleaning cuts, clearly shows the typical azimuthal signature of BIB, as described in sections 4 and 7.1. Here, the total number of selected events is $\sim 694000$. 


\begin{tabular}{|l|l|l|}
\hline selection & total number of events & non-collision background \\
\hline monojet selection & $\sim 694000$ & $\sim 557000$ \\
after Medium jet cleaning & $\sim 134000$ & $\sim 7000$ \\
\hline after $f_{\text {ch }}$ and $f_{\text {EM }}$ cuts & 124704 & $575 \pm 60($ stat $) \pm 57($ sys $)$ \\
\hline
\end{tabular}

Table 5. Number of events in the monojet signal region before and after the cleaning cuts. Non-collision background levels are also indicated. The last row corresponds to the monojet analysis signal region where the non-collision background is the BIB contamination determined using the two-sided method. In the other two cases, the estimate is based on the jet selection inefficiency evaluated in Monte Carlo simulations.

The Medium jet cleaning reduces the amount of BIB significantly by removing $\sim 560000$ events from the sample, which is $\sim 80 \%$ of the original sample size. However, as discussed in section 8.1.2, it identifies $99.5 \%$ of the fake jets, which means that a certain residual amount of BIB after the cleaning is still expected. Indeed, the contamination from BIB in the remaining $\sim 134000$ events after this cleaning is visible as a slight excess at $\phi=0$ and $\phi=\pi$. Therefore, even stronger cleaning is needed and the additional cuts on the leading jet charged particle fraction and electromagnetic energy fraction are applied. The resulting $\phi$ distribution looks flat which demonstrates the rejection power of these cleaning cuts. The flat $\phi$ distribution suggests that the sample is dominated by physics processes as indicated in figure 22. The number of events in the monojet analysis signal region, i.e. after the monojet selection with all the cleaning cuts, is 124704 which corresponds to $\sim 18 \%$ of the size of the original sample without any cleaning applied. These selected events correspond mainly to physics processes but there may still be a small fraction of BIB events left.

The dominant Standard Model backgrounds ( $Z$ and $W$ boson plus jet production) are estimated in a data-driven way in dedicated control regions. Multi-jet backgrounds are also estimated from data, while the diboson and top-quark backgrounds are obtained from Monte Carlo simulations. Since the monojet analysis searches for rare events (beyond Standard Model physics), even the smallest backgrounds need to be estimated accurately in order to quantify how many of the events may be due to new phenomena. The two-sided method, described in section 7.2, is completely independent of the jet cleaning criteria applied in the monojet signal region selection, and is used to quantify the residual number of BIB events present in the 124704 monojet signal region events. As shown in table 5, the method estimates the BIB level to be $575 \pm 60$ (stat) \pm 57 (sys) events. This residual background is also indicated in figure 31. As expected, the distribution of the leading jet charged particle fraction shows that a majority of the events tagged as BIB have leading jets without tracks pointing to them.

A Monte Carlo study reveals that the Medium cleaning selection criteria applied to all jets with $p_{\mathrm{T}}^{\text {jet }}>20 \mathrm{GeV}$ removes $\sim 7 \%$ of physics events passing the monojet selection. Introducing the additional cuts on the leading jet $f_{\mathrm{ch}}$ and $f_{\mathrm{EM}}$ reduces the number of physics events in the Monte Carlo samples by an additional $\sim 2 \%$. These estimates for the jet selection inefficiency can be used to determine the number of non-collision background events in the sample after the monojet selection with and without the Medium cleaning selection criteria applied (see table 5).

The total number of non-collision background events in the data sample just after the monojet selection is $\sim 557000$, which corresponds to $80 \%$ of the sample size. After applying all the cleaning cuts the number of BIB events in the sample is reduced to 575 , corresponding to a rejection power of $\sim 10^{3}$ for this analysis. 

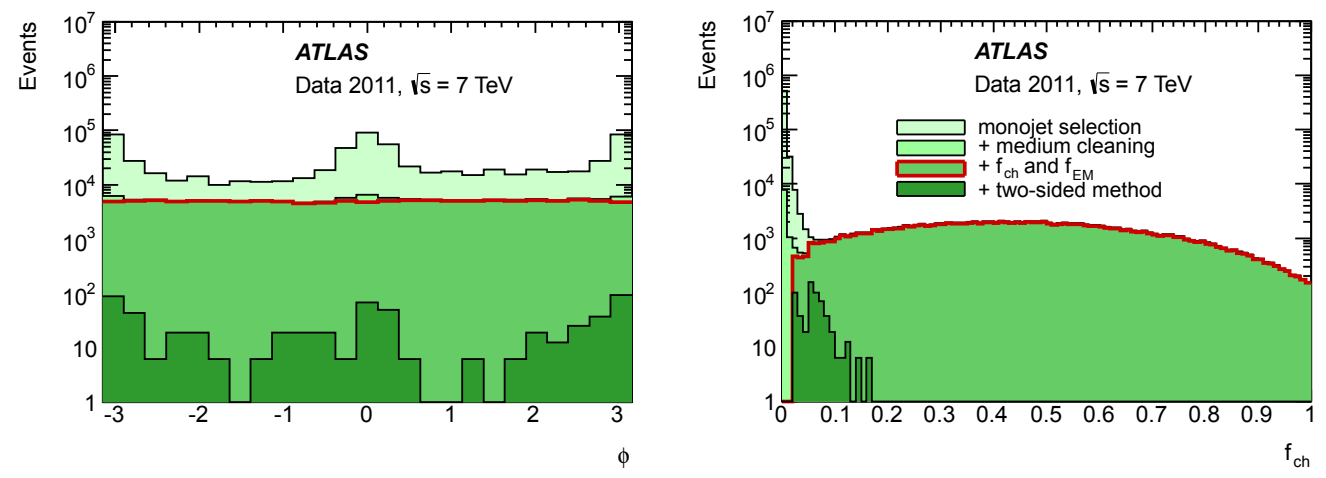

Figure 31. Azimuthal distribution (left) and the charged particle fraction (right) of the leading jet in the monojet analysis signal region before and after the cleaning cuts. The monojet analysis signal region events are emphasised by the red line. The residual level of BIB as estimated by the two-sided method is also shown.
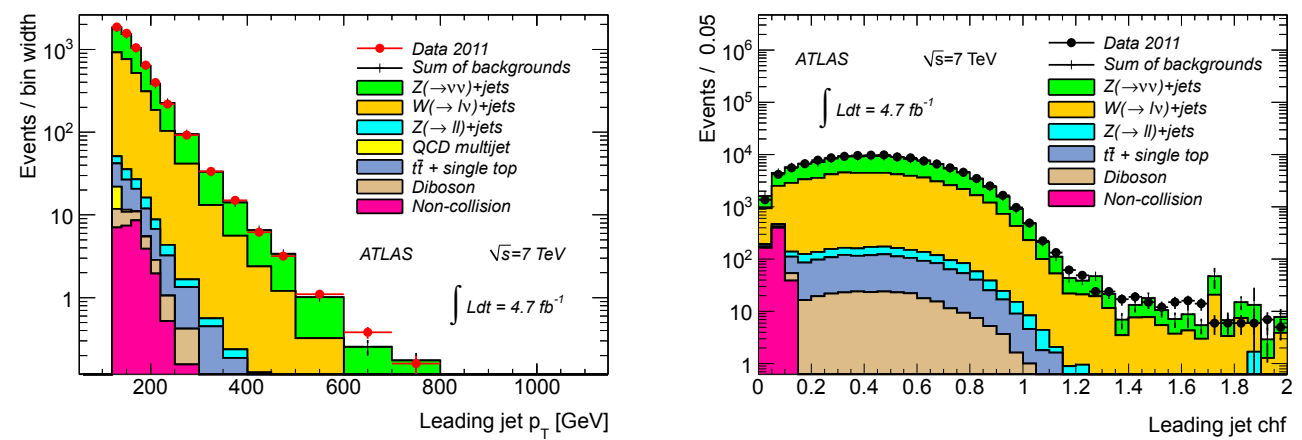

Figure 32. Transverse momentum (left) and the charged particle fraction (right) of the leading jet in the monojet analysis signal region. The non-collision background is the BIB evaluated by the two-sided method.

Finally, figure 32 shows the leading jet $p_{\mathrm{T}}$ distribution and the leading jet charged particle fraction distribution for the monojet signal events together with various sources of Standard Model backgrounds. The residual BIB, which amounts to only $0.5 \%$ of the signal region events, is also illustrated in the figure. The other events in the monojet signal region sample of 124704 events are in agreement with the background estimates for Standard Model processes. No evidence for physics beyond the Standard Model is found in the 2011 data. All the fake jets tagged by the twosided method in this analysis have $f_{\text {ch }}<0.2$ and have a $p_{\mathrm{T}}^{\text {jet }}$ lower than $300 \mathrm{GeV}$. These events are typically BIB muons overlaid on top of a minimum bias process. An example of such a BIB event in the monojet analysis signal region is shown in figure 33 where a BIB muon travels in the $\mathrm{A} \rightarrow \mathrm{C}$ direction leaving hits in the CSC detectors on both sides of ATLAS. A LAr calorimeter cluster stretched along the $z$-axis is seen in-between, leading to a fake jet with $p_{\mathrm{T}}^{\text {jet }} \sim 270 \mathrm{GeV}$ with the corresponding missing transverse momentum in the opposite direction. No collision tracks point towards this jet. The energy of BIB muons can be up to the TeV level, and a few cases have been seen in data where the energy deposition of such a BIB muon has been reconstructed as a jet with $p_{\mathrm{T}}^{\mathrm{jet}}>1 \mathrm{TeV}$. 


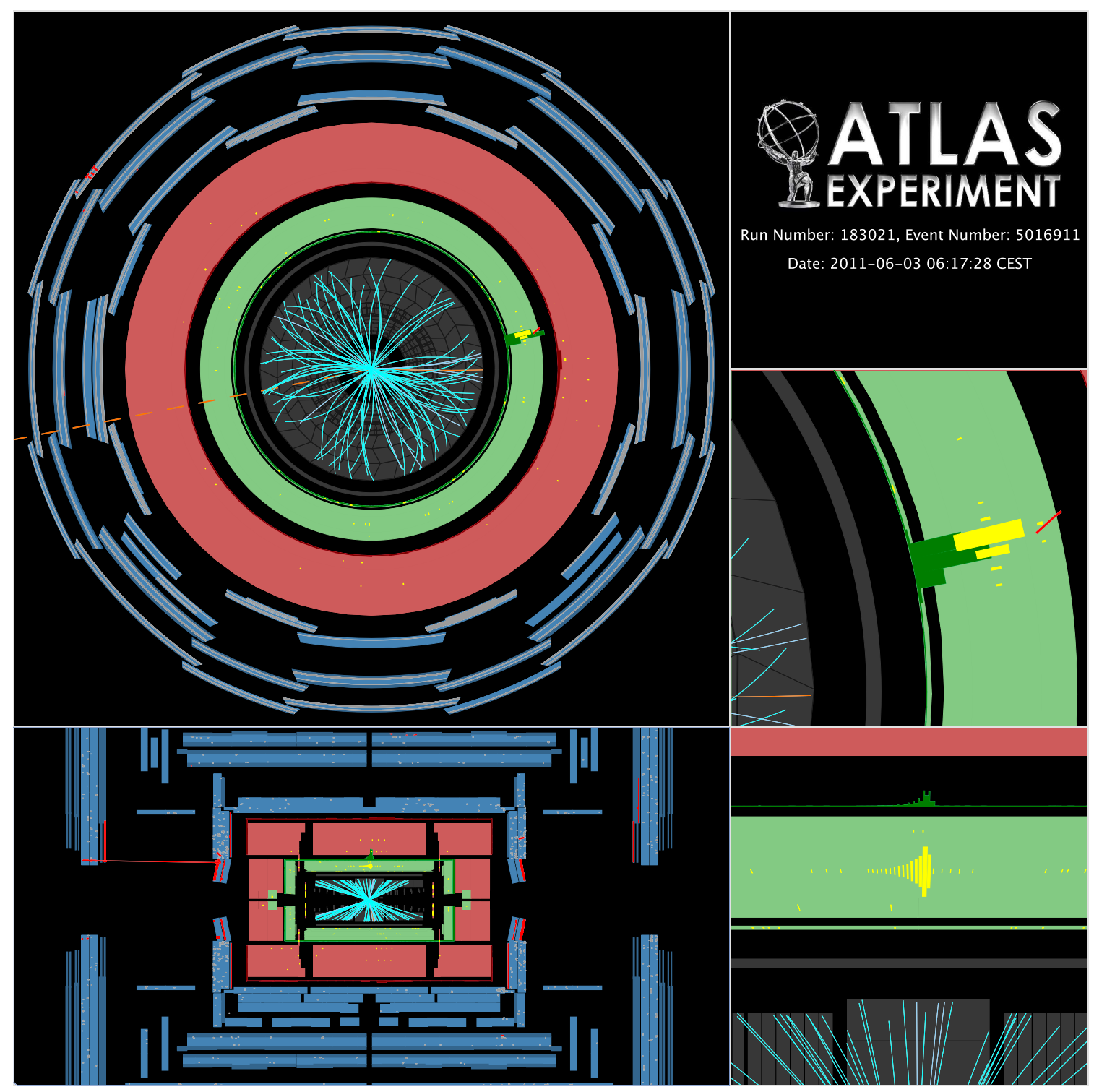

Figure 33. Example of an event in the monojet analysis signal region with a BIB muon entering from the right and causing a fake jet. In the longitudinal projection (bottom left), CSC chambers with hits (highlighted in red) are seen on both sides. LAr calorimeter cells (yellow) in-between contain large energy (green towers) that forms a fake jet. A muon track (red line) parallel to the $z$-axis is reconstructed on side $\mathrm{C}$. The transverse projection (top left) shows $E_{\mathrm{T}}^{\text {miss }}$ (dashed line) opposite to the fake jet. The reconstructed tracks (blue) in the inner tracking detector do not point towards the fake jet. A detailed view (middle right) shows that the calorimeter cells and the muon track are aligned in $\phi$. Focusing on the LAr energy depositions in the longitudinal projection (bottom right) reveals that the fake jet consists of a cluster elongated in the $z$ direction.

A tighter cut on the leading jet charged particle fraction could clearly remove non-collision background events even further. The two-sided method allows studies of the efficiency and the misidentification probability of different cleaning cuts. Such studies, using Monte Carlo simulation samples, reveal that tighter cleaning cuts also significantly reduce the signal acceptance, which is 
not desired in searches. The set of cleaning cuts used in the monojet analysis is a balance between large background rejection and small physics signal suppression.

Since the efficiency of the jet charged particle fraction cut is expected to decrease with increasing pile-up, the independent methods of BIB removal described here are expected to become more important in LHC runs after 2011.

\subsection{Summary of jet cleaning techniques}

The selection method based on jet observables to remove non-collision backgrounds is particularly powerful and widely used in ATLAS physics analysis. The Looser criteria already provide good background rejection, while having a negligible loss of efficiency for jets originating from protonproton collisions. The collision-jet selection efficiency is better than $99.8 \%$ for $p_{\mathrm{T}}^{\mathrm{jet}}>20 \mathrm{GeV}$ and its performance is well reproduced by the Monte Carlo simulation. When larger rejection factors of non-collision backgrounds are needed, further selection criteria based on the electromagnetic fraction and the charged particle fraction of the jets can be applied. Such tighter cleaning cuts have been successfully applied in new physics searches, for instance the monojet signatures search. There, the topology of the signal region events is similar to the signatures of jets due to noncollision backgrounds, and it has been shown that $\sim 80 \%$ of the selected data come from noncollision backgrounds if no cleaning cuts are applied. Dedicated cleaning reduces the non-collision background contamination to $0.5 \%$, where the estimate of the residual BIB level is carried out using the methods described in section 7.2, which are independent of the cleaning cuts.

\section{Conclusions}

During the 2011 proton run the LHC delivered more than $5 \mathrm{fb}^{-1}$ of luminosity, of which about $4.7 \mathrm{fb}^{-1}$ is usable for physics analyses. The number of colliding bunches increased during the year from a few hundred to 1331. Each physics fill of the LHC also contained on the order of 50 unpaired, i.e. non-colliding, bunches to monitor the beam induced background (BIB). The events in those unpaired bunches were triggered by dedicated algorithms and stored in a special background stream at a rate of a few $\mathrm{Hz}$.

Due to the large event rate, the Level-1 trigger rates before prescaling allowed detailed monitoring of backgrounds, while the recorded events formed the basis for developing dedicated background tagging tools to be used in physics analyses.

The main detector used for beam-gas monitoring in ATLAS is the Beam Conditions Monitor (BCM), located very close to the beam-line. A special background-like trigger was implemented for BCM hits, which selected only events with an early hit on one side and an in-time hit on the other side of the ATLAS Interaction Point (IP). The rates of this trigger are shown to correlate very well with residual gas pressure close to the experiment (the pressure measured at $|z|=22 \mathrm{~m}$ ), but have much less sensitivity to beam losses further away, i.e. pressure at $z=58 \mathrm{~m}$.

The BCM also provides a collision-like trigger, i.e. an in-time coincidence on both sides of the IP. The rates from this trigger are used to study the ghost charge distribution by looking for collisions of protons in unpaired bunches with protons in nominally empty bunches. These studies reveal that non-negligible ghost charge can extend as far as $150 \mathrm{~ns}$ from the filled bunches. This result is supported by similar findings for the Level-1 $\mathrm{J} 10$ (jet with $p_{\mathrm{T}}>10 \mathrm{GeV}$ ) trigger rates. 
In order to gain a deeper understanding of BIB sources and formation, dedicated simulations have been performed [9]. The main results of these simulations are presented in this paper and characteristic features of the BIB, such as radial and azimuthal distributions, are shown. Some of these characteristic features have been observed in 2011 data as well.

The various ATLAS sub-detectors allow accurate studies of the BIB to be performed. A particularly well-suited detector for studying BIB at small radii is the ATLAS Pixel detector. Since the Pixel barrel is coaxial with the beam-line and BIB tracks are predominantly parallel to the beam, a characteristic feature of BIB events in the Pixel detector is the presence of elongated clusters with large total charge deposition. This feature has been used to develop an algorithm for tagging BIB events. Comparison of data and simulations indicates very good agreement for both collisions and BIB. The tagging tool has been used to produce background samples that show that the BIB rate in the Pixel detector correlates very well with the residual pressure at $|z|=22 \mathrm{~m}$. This shows that the Pixel detector, like the BCM, is sensitive mainly to beam-gas events close to the detector. However, the background data also show a slight $\phi$-asymmetry. The BIB simulations suggest that such an asymmetry is created by bending in the magnets of the inner triplet and beyond. Thus a fraction of the background seen by the Pixel detector seems to originate from a larger distance.

The main impact of BIB on physics analyses is the production of fake jets due to radiative energy losses of high-energy muons passing through the calorimeters. This affects mainly the analyses relying on large $E_{\mathrm{T}}^{\text {miss }}$ signatures. The simulations indicate that such muons have to originate far from the detector $(>100 \mathrm{~m})$ in order to reach the calorimeter radii. In addition they are predicted to show a very pronounced $\phi$-asymmetry with a strong preference for muons to be in the horizontal plane. Such an asymmetry is clearly seen in the distribution of fake-jet candidates. A special tool, based on identifying the incoming/outgoing muon in the CSC and the inner MDT endcap muon chambers, has been developed to remove such events from physics analysis. This tool comprises several algorithms, yielding different efficiency and mis-identification probabilities. In addition to using the position and direction information from the muon detectors (both polar and azimuthal) it also uses timing information of both the muon detectors and the calorimeter.

The standard jet cleaning algorithms used in ATLAS to reject non-collision background events have been summarised and their application in the monojet signatures search has been presented. It has been shown that after the jet cleaning criteria are applied, the event sample still contains BIB events, which are identified by a special analysis tool and taken into account in the background estimates in this analysis. Without this dedicated cleaning, BIB events would represent a serious background for some searches for new phenomena.

\section{A Alternative methods for BIB identification in the calorimeters}

This appendix outlines two alternative methods for BIB identification in the calorimeters in addition to those described in section 7.2. The first method uses the time signature of energy depositions due to BIB in the TileCal. The other checks the shape and orientation of a calorimeter cluster in order to differentiate between BIB and collision products. Both methods are presently under study.

\section{A.1 Beam background signatures in the Tile calorimeter}

The timing measurements with 1 ns resolution, and spatial information for the measured energy depositions of the TileCal provide an analysis tool capable of reconstructing muons which may 

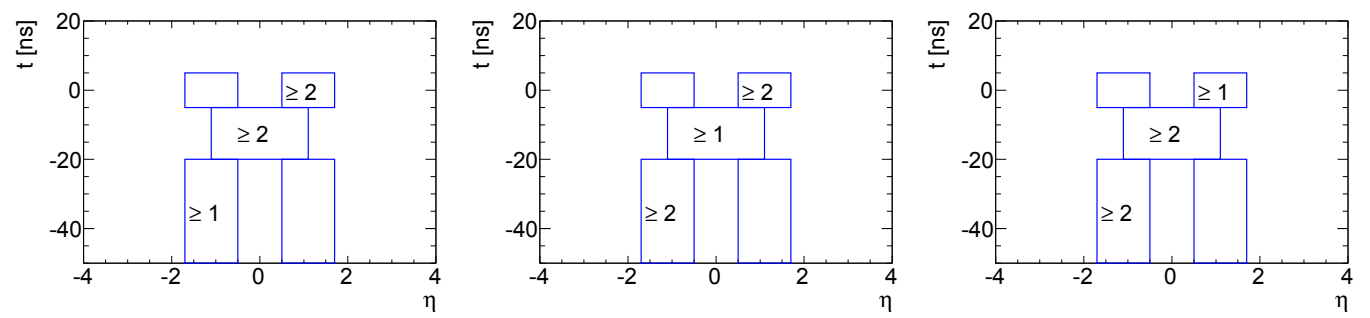

Figure 34. Selection criteria for the TileCal muon filter. The blue rectangles correspond to the $\eta-t$ regions used by the TileCal muon filter to select the events. The numbers correspond to the minimum number of selected clusters required in each region.

originate from BIB. A possible signature of such muons is a series of aligned energy depositions parallel to the beam direction, starting on one side of the TileCal and propagating to the other. The time measurement of the energy deposits has to be consistent with the hypothesis of a particle travelling parallel to the beam direction at the speed of light, while having roughly the same azimuthal angle $(\phi)$ in the detector. The criteria used to identify such distinct patterns are the following:

- Select calorimeter clusters with a fraction of energy in the TileCal of at least $90 \%$.

- The TileCal is divided in 64 overlapping slices in $\phi$, such that the $n^{\text {th }}$ slice covers $\phi \in$ $\left[n \frac{\pi}{32}, n \frac{\pi}{32}+\frac{\pi}{16}\right]$. The width of each $\phi$ slice, $\frac{\pi}{16}$, corresponds to two consecutive TileCal modules, which define the angular resolution of the TileCal in $\phi$. In each $\phi$ slice, clusters are selected if their pseudorapidity and time measurement are compatible with the hypothesis of a particle travelling parallel to the beam axis.

- A minimum number of selected clusters is required to tag a BIB muon candidate and it is required that they have a specific pattern in $\eta-t$. Figure 34 illustrates the $\eta-t$ regions that are defined to tag BIB muons and shows the minimum cluster multiplicity requirement in each of the regions along the muon path. No additional selection criterion is applied, i.e. these $\eta-t$ regions have no segmentation in the radial direction. Three sets of cuts on the number of clusters in each region are defined in the figure. The illustrated selection criteria apply to muon background travelling in the $\mathrm{C} \rightarrow \mathrm{A}$ direction. For the $\mathrm{A} \rightarrow \mathrm{C}$ direction, the diagonal of the $\eta-t$ regions is reversed.

An example of a BIB event tagged in an unpaired bunch is shown in figure 35 .

The efficiency of the selection criteria is evaluated in the data from unpaired bunches, requiring exactly one jet with $p_{\mathrm{T}}^{\text {jet }}>30 \mathrm{GeV},|\eta|<1.5$. A timing cut of $t<-5 \mathrm{~ns}$ is applied in order to reduce the contamination from ghost collisions in the unpaired bunch data, and $90 \%$ of the jet energy should belong to TileCal channels in order to ensure that the BIB particle deposits its energy in the TileCal. Since collision data samples are always contaminated by BIB events, the mis-identification rate of the TileCal muon filter is estimated with a multijet Monte Carlo sample that reproduces the pile-up conditions of the data. The BIB-enriched and Monte Carlo samples described above are composed of 2101 and $1.4 \cdot 10^{6}$ events, respectively. The efficiency for the selection criteria depicted in figure 34 is about $12 \%$, and the mis-identification rate is about $2 \cdot 10^{-4}$. By requiring only one hit in each of the relevant $\eta-t$ regions, the efficiency is higher by about a factor three and 


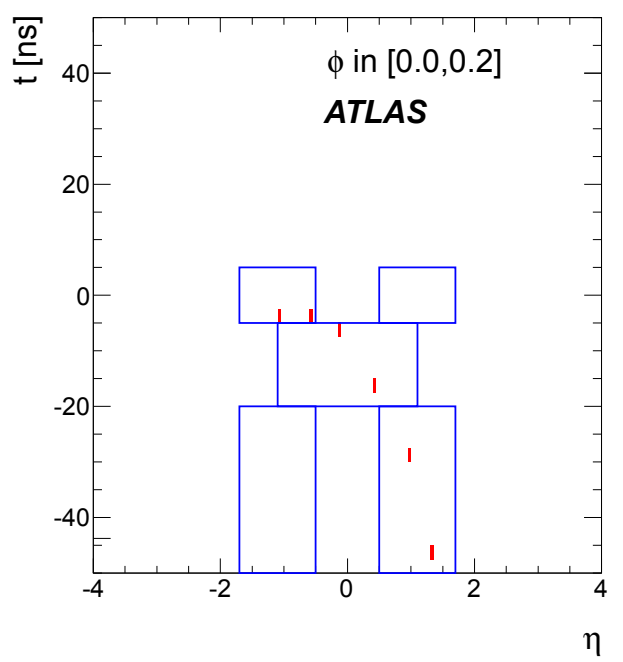

Figure 35. Example of an event selected by the TileCal muon filter in unpaired bunches. The clusters are shown in red marks and belongs to the same $\phi$ slice.

the mis-identification rate increases by more than two orders of magnitude. If the minimum number of required hits in the regions is increased to two, the mis-identification rate drops to about $10^{-6}$ and the selection efficiency decreases to only about $1 \%$. The relatively low efficiency can be explained by the fact that the selection criteria require the muon to cross the calorimeter completely from side to side. Muons that enter on one side and are stopped inside the calorimeter are not tagged, but they contribute to the inefficiency of the method.

\section{A.2 Cluster shape}

Because BIB muons travel parallel to the beam-pipe, their cluster shapes in the calorimeter are different from those generated by collisions. The particle shower develops mainly along the $z$ direction for BIB, whereas for collisions it develops in the direction from the interaction point. In order to distinguish between BIB and collision products based on the cluster shape, one can compare the standard deviations of the $z$ and $r$ positions of the cells contained within a cluster. The ratio

$$
\frac{\sigma_{r}}{\sigma_{z}}=\frac{\sum\left(r_{\text {cell }}-r_{\text {clus }}\right)^{2}}{\sum\left(z_{\text {cell }}-z_{\text {clus }}\right)^{2}}
$$

is defined, where $z_{\text {cell }}, r_{\text {cell }}$ and $z_{\text {clus }}, r_{\text {clus }}$ are the positions of cells and clusters, respectively. Only the cells with a well-measured time and an energy deposition above $100 \mathrm{MeV}$ are considered in the sum in order to suppress noise. Figure 36 compares the ratio of standard deviations in $z$ and $r$, $\sigma_{r} / \sigma_{z}$, for BIB muon data from unpaired bunches with simulated collision data. The distributions motivate a cut on $\sigma_{r} / \sigma_{z}<0.15$ to select BIB muons. It can be seen that some of the clusters from collisions also satisfy the cut on $\sigma_{r} / \sigma_{z}<0.15$. Given the large number of clusters per event, this leads to non-negligible mis-tagging rates. In order to reduce mis-identification of collisions due to this fact, selection criteria based on other quantities need to be applied as well. 


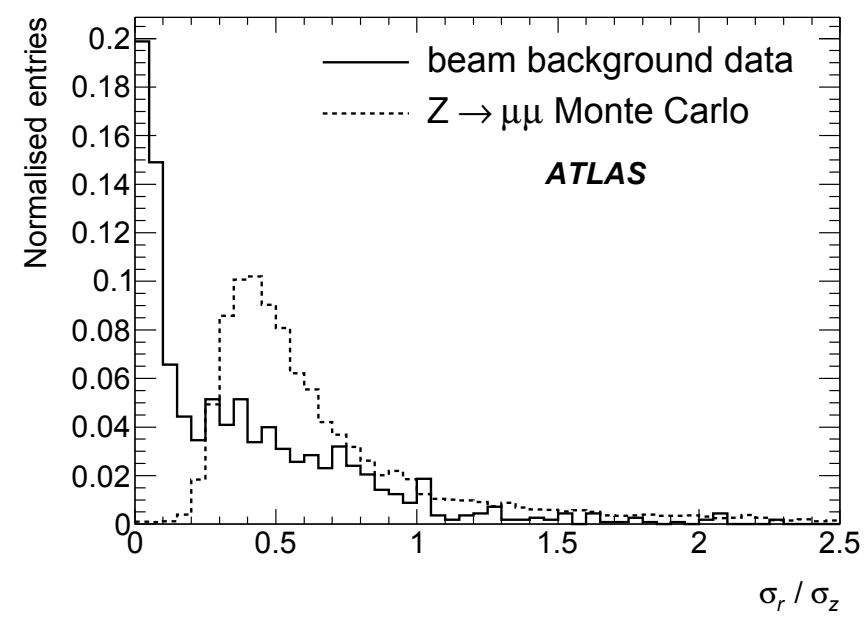

Figure 36. Ratio of the standard deviation of $r$ and $z$ position of the cells contained within a cluster in unpaired bunches (solid) and simulated collision events (dashed). Taken from [29].

\section{Acknowledgments}

We thank CERN for the very successful operation of the LHC, as well as the support staff from our institutions without whom ATLAS could not be operated efficiently.

We thank, especially, G. Bregliozzi and G. Lanza from the LHC Vacuum group for providing us with pressure maps and explanations. We are also grateful for the help of the FLUKA team at CERN, who contributed to the geometry models of the LHC interaction region simulations.

We acknowledge the support of ANPCyT, Argentina; YerPhI, Armenia; ARC, Australia; BMWF and FWF, Austria; ANAS, Azerbaijan; SSTC, Belarus; CNPq and FAPESP, Brazil; NSERC, NRC and CFI, Canada; CERN; CONICYT, Chile; CAS, MOST and NSFC, China; COLCIENCIAS, Colombia; MSMT CR, MPO CR and VSC CR, Czech Republic; DNRF, DNSRC and Lundbeck Foundation, Denmark; EPLANET, ERC and NSRF, European Union; IN2P3-CNRS, CEA-DSM/IRFU, France; GNSF, Georgia; BMBF, DFG, HGF, MPG and AvH Foundation, Germany; GSRT and NSRF, Greece; ISF, MINERVA, GIF, DIP and Benoziyo Center, Israel; INFN, Italy; MEXT and JSPS, Japan; CNRST, Morocco; FOM and NWO, Netherlands; BRF and RCN, Norway; MNiSW, Poland; GRICES and FCT, Portugal; MERYS (MECTS), Romania; MES of Russia and ROSATOM, Russian Federation; JINR; MSTD, Serbia; MSSR, Slovakia; ARRS and MIZŠ, Slovenia; DST/NRF, South Africa; MICINN, Spain; SRC and Wallenberg Foundation, Sweden; SER, SNSF and Cantons of Bern and Geneva, Switzerland; NSC, Taiwan; TAEK, Turkey; STFC, the Royal Society and Leverhulme Trust, United Kingdom; DOE and NSF, United States of America.

The crucial computing support from all WLCG partners is acknowledged gratefully, in particular from CERN and the ATLAS Tier-1 facilities at TRIUMF (Canada), NDGF (Denmark, Norway, Sweden), CC-IN2P3 (France), KIT/GridKA (Germany), INFN-CNAF (Italy), NL-T1 (Netherlands), PIC (Spain), ASGC (Taiwan), RAL (UK) and BNL (USA) and in the Tier-2 facilities worldwide. 


\section{References}

[1] L. Evans and P. Bryant, LHC Machine, 2008 JINST 3 S08001.

[2] LHC design Report, http://lhc.web.cern.ch/lhc/lhc-designreport.html.

[3] H. Wiedemann, Particle Accelerator Physics, third edition, Springer (2007).

[4] M. Bosman et al., Estimation of Radiation Background, Impact on Detectors, Activation and Shielding Optimization in ATLAS, ATL-GEN-2005-001 (2005).

[5] R. Assmann, Collimation for the LHC High Intensity Beams, Proceedings of HB2010, Morschbach, Switzerland, 2010, http://epaper.kek.jp/HB2010/index.htm.

[6] R. Assmann et al., Collimators and Beam Absorbers for Cleaning and Machine Protection, Proceedings of the LHC Project Workshop, Chamonix XIV, 2005, pg. 2016, http://indico.cern.ch/conferenceDisplay.py?confId $=044$.

[7] R. Assmann et al., The Final Collimation System for the LHC, Proceedings of EPAC 2006, Edinburgh, Scotland, 2006, pg. 986, http://accelconf.web.cern.ch/accelconf/e06/.

[8] G. Valentino et al., Multi-turn losses and cleaning in 2011 and 2012, Proceedings of the 2011 LHC beam operation workshop, Evian, France, 2011, http://indico.cern.ch/conferenceDisplay.py?confId=155520.

[9] R.Bruce et al., Sources of machine-induced background in the ATLAS and CMS detectors at the CERN Large Hadron Collider, to be submitted to Nucl. Instrum. Meth. A.

[10] C. Benvenuti et al., Decreasing surface outgassing by thin film getter coatings, Vacuum 50 (1998) 57.

[11] R. Cimino et al., Can low energy electrons affect high energy physics accelerators?, Phys. Rev. Lett. 93 (2004) 014801.

[12] G. Bregliozzi, G. Lanza, V. Baglin and J.M. Jiminez, Vacuum pressure observations during the 2011 proton run, Proceedings of the 2011 LHC beam operation workshop, Evian, France, 2011, http://indico.cern.ch/conferenceDisplay.py?confId=155520.

[13] A. Rossi, G. Rumolo and F. Zimmermann, A Simulation Study of Electron Cloud in the Experimental Regions of the LHC, Proceedings of EPAC 2002, Paris, France, 2002, http://accelconf.web.cern.ch/accelconf/e02/default.htm.

[14] ATLAS collaboration, The ATLAS Experiment at the CERN Large Hadron Collider, 2008 JINST 3 S08003.

[15] V. Cindro et al., The ATLAS beam conditions monitor, 2008 JINST 3 P02004.

[16] M. Cacciari, G.P. Salam and G. Soyez, The Anti-k(t) jet clustering algorithm, JHEP 04 (2008) 063 [arXiv:0802.1189].

[17] W. Lampl et al., Calorimeter Clustering Algorithms: Description and Performance, ATL-LARG-PUB-2008-002 (2008).

[18] ATLAS collaboration, Readiness of the ATLAS Liquid Argon Calorimeter for LHC Collisions, Eur. Phys. J. C 70 (2010) 723 [arXiv:0912.2642].

[19] A. Drozhdin, M. Huhtinen and N. Mokhov, Accelerator related background in the CMS detector at LHC, Nucl. Instrum. Meth. A 381 (1996) 531.

[20] A.I. Drozhdin, N.V. Mokhov and S.I. Striganov, Beam Losses and Background Loads on Collider Detectors Due to Beam-Gas Interactions in the LHC, Proceedings of PAC09, Vancouver, Canada, April 2009. Fermilab-Conf-09-172 (2009). 
[21] R. Bruce et al., Machine-induced showers entering the ATLAS and CMS detectors in the LHC, Proceedings of IPAC'11, San Sebastian, Spain 2011, http://accelconf.web.cern.ch/accelconf/IPAC2011/index.htm.

[22] G. Bregliozzi, G.Lanza, V. Baglin and J. M. Jiminez, Vacuum stability and residal gas density estimation for the vacuum chamber upgrade of the ATLAS interaction region at the Large Hadron Collider, Vacuum 86 (2012) 1682.

[23] N.V. Mokhov and T. Weiler, Machine-induced backgrounds: their origin and loads on ATLAS/CMS, Proceedings of the LHC workshop on Experimental Conditions and Beam-induced Detectors Backgrounds, CERN-2009-003 (2009).

[24] F. Schmidt, SixTrack Version 4.2.16, Single Particle Tracking Code Treating Transverse Motion with Synchrotron Oscillations in a Symplectic Manner, Report CERN/SL/94-56-AP (1994).

[25] A. Ferrari, P. R. Sala, A. Fasso and J. Ranft, FLUKA: a multi-particle transport code, Report CERN-2005-10 (2005);

G. Battistoni at al., The FLUKA code: Description and benchmarking, Proceedings of Hadronic Shower Simulation Workshop 2006, Fermilab September 6-8 2006, AIP Conf. Proc. 896 (2007) 31.

[26] Particle Data Group collaboration, J. Beringer et al., 2012 Review of Particle Physics, section 30.6, Phys. Rev. D 66 (2002) 204.

[27] B. Trocmé, The Atlas Liquid Argon Calorimeter: Commissioning with Cosmic Muons and First LHC Beams, Proceedings of the 11th Topical Seminar on Innovative Particle and Radiation Detectors, Siena, Italy, October 1-4 2008, Nucl. Phys. (Proc. Suppl.) 197 (2009) 139.

[28] S. Ask, et al., The ATLAS central level-1 trigger logic and TTC system, 2008 JINST 3 P08002.

[29] ATLAS collaboration, Non-collision backgrounds as measured by the ATLAS detector during the 2010 proton-proton run, ATLAS-CONF-2011-137 (2011).

[30] ATLAS collaboration, Luminosity Determination in pp Collisions at $\sqrt{s}=7 \mathrm{TeV}$ Using the ATLAS Detector at the LHC, Eur. Phys. J. C 71 (2011) 1630 [arXiv:1101.2185].

[31] A. Alici et al., Study of the LHC ghost charge and satellite bunches for luminosity calibration., CERN-ATS-Note-2012-029 (2012).

[32] A. Jeff, A Longitudinal Density Monitor for the LHC, Ph.D. thesis, Department of Physics, University of Liverpool U.K. (2012).

[33] T. Sjöstrand, S. Mrenna and P.Z. Skands, PYTHIA 6.4 Physics and Manual, JHEP 05 (2006) 026 [hep-ph/0603175].

[34] ATLAS collaboration, Search for dark matter candidates and large extra dimensions in events with a jet and missing transverse momentum with the ATLAS detector, CERN-PH-EP-2012-210 (2012).

[35] ATLAS collaboration, Performance of Missing Transverse Momentum Reconstruction in Proton-Proton Collisions at 7 TeV with ATLAS, Eur. Phys. J. C 72 (2012) 1844 [arXiv: 1108. 5602].

[36] ATLAS collaboration, Performance of the ATLAS Trigger System in 2010, Eur. Phys. J. C 72 (2012) 1849 [arXiv: 1110.1530].

[37] ATLAS collaboration, Search for new phenomena with the monojet and missing transverse momentum signature using the ATLAS detector in $\sqrt{s}=7 \mathrm{TeV}$ proton-proton collisions, CERN-PH-EP-2011-090 (2011). 


\section{The ATLAS collaboration}

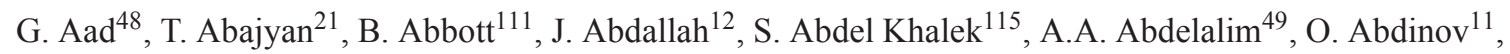
R. Aben ${ }^{105}$, B. Abi ${ }^{112}$, M. Abolins ${ }^{88}$, O.S. AbouZeid ${ }^{158}$, H. Abramowicz ${ }^{153}$, H. Abreu ${ }^{136}$, B.S. Acharya ${ }^{164 a, 164 b, a}$, L. Adamczyk ${ }^{38}$, D.L. Adams ${ }^{25}$, T.N. Addy ${ }^{56}$, J. Adelman ${ }^{176}$, S. Adomeit ${ }^{98}$, P. Adragna ${ }^{75}$, T. Adye ${ }^{129}$, S. Aefsky ${ }^{23}$, J.A. Aguilar-Saavedra ${ }^{124 b, b}$, M. Agustoni ${ }^{17}$, M. Aharrouche ${ }^{81}$, S.P. Ahlen ${ }^{22}$, F. Ahles ${ }^{48}$, A. Ahmad ${ }^{148}$, M. Ahsan ${ }^{41}$, G. Aielli ${ }^{133 a, 133 b}$, T. Akdogan ${ }^{19 a}$, T.P.A. Åkesson ${ }^{79}$, G. Akimoto ${ }^{155}$, A.V. Akimov ${ }^{94}$, M.S. Alam ${ }^{2}$, M.A. Alam ${ }^{76}$, J. Albert ${ }^{169}$, S. Albrand ${ }^{55}$, M. Aleksa ${ }^{30}$, I.N. Aleksandrov ${ }^{64}$, F. Alessandria ${ }^{89 a}$, C. Alexa $^{26 a}$, G. Alexander ${ }^{153}$, G. Alexandre ${ }^{49}$, T. Alexopoulos ${ }^{10}$, M. Alhroob ${ }^{164 a, 164 c}$, M. Aliev ${ }^{16}$, G. Alimonti ${ }^{89 a}$, J. Alison ${ }^{120}$, B.M.M. Allbrooke ${ }^{18}$, P.P. Allport ${ }^{73}$,

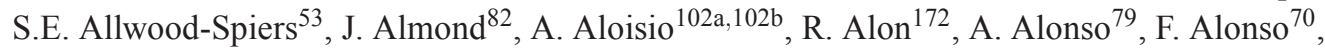
A. Altheimer ${ }^{35}$, B. Alvarez Gonzalez ${ }^{88}$, M.G. Alviggi ${ }^{102 a, 102 b}$, K. Amako ${ }^{65}$, C. Amelung ${ }^{23}$, V.V. Ammosov ${ }^{128, *}$, S.P. Amor Dos Santos ${ }^{124 a}$, A. Amorim ${ }^{124 a, c}$, N. Amram ${ }^{153}$, C. Anastopoulos ${ }^{30}$, L.S. Ancu ${ }^{17}$, N. Andari ${ }^{115}$, T. Andeen ${ }^{35}$, C.F. Anders ${ }^{58 b}$, G. Anders ${ }^{58 a}$, K.J. Anderson ${ }^{31}$, A. Andreazza ${ }^{89 a, 89 b}$, V. Andrei ${ }^{58 a}$, M-L. Andrieux ${ }^{55}$, X.S. Anduaga ${ }^{70}$, S. Angelidakis ${ }^{9}$, P. Anger ${ }^{44}$, A. Angerami ${ }^{35}$, F. Anghinolfi ${ }^{30}$, A. Anisenkov ${ }^{107}$, N. Anjos ${ }^{124 a}$, A. Annovi ${ }^{47}$, A. Antonaki ${ }^{9}$, M. Antonelli ${ }^{47}$, A. Antonov ${ }^{96}$, J. Antos ${ }^{144 b}$, F. Anulli1 ${ }^{132 a}$, M. Aoki ${ }^{101}$, S. Aoun ${ }^{83}$, L. Aperio Bella ${ }^{5}$, R. Apolle ${ }^{18, d}$, G. Arabidze ${ }^{88}$, I. Aracena ${ }^{143}$, Y. Arai ${ }^{65}$, A.T.H. Arce ${ }^{45}$, S. Arfaoui ${ }^{148}$, J-F. Arguin ${ }^{93}$,

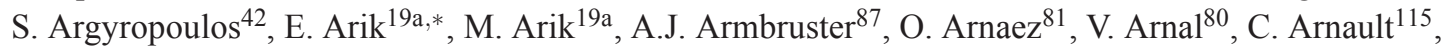

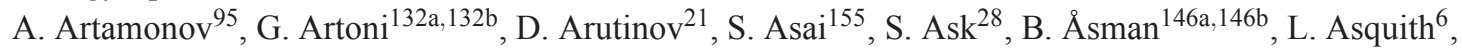
K. Assamagan ${ }^{25, e}$, A. Astbury ${ }^{169}$, M. Atkinson ${ }^{165}$, B. Aubert ${ }^{5}$, E. Auge ${ }^{115}$, K. Augsten ${ }^{126}$, M. Aurousseau ${ }^{145 a}$, G. Avolio ${ }^{30}$, R. Avramidou ${ }^{10}$, D. Axen ${ }^{168}$, G. Azuelos ${ }^{93, f}$, Y. Azuma ${ }^{155}$, M.A. Baak ${ }^{30}$, G. Baccaglioni89a , C. Bacci ${ }^{134 a, 134 b}$, A.M. Bach ${ }^{15}$, H. Bachacou ${ }^{136}$, K. Bachas ${ }^{30}$, M. Backes ${ }^{49}$, M. Backhaus ${ }^{21}$, J. Backus Mayes ${ }^{143}$, E. Badescu ${ }^{26 a}$, P. Bagnaia ${ }^{132 a, 132 b}$, S. Bahinipati ${ }^{3}$, Y. Bai ${ }^{33 a}$, D.C. Bailey ${ }^{158}$, T. Bain ${ }^{35}$, J.T. Baines ${ }^{129}$, O.K. Baker ${ }^{176}$, M.D. Baker ${ }^{25}$, S. Baker ${ }^{77}$, P. Balek ${ }^{127}$, E. Banas ${ }^{39}$, P. Banerjee ${ }^{93}$, Sw. Banerjee ${ }^{173}$, D. Banfi ${ }^{30}$, A. Bangert ${ }^{150}$, V. Bansal ${ }^{169}$, H.S. Bansil ${ }^{18}$, L. Barak $^{172}$, S.P. Baranov ${ }^{94}$, A. Barbaro Galtieri ${ }^{15}$, T. Barber ${ }^{48}$, E.L. Barberio ${ }^{86}$, D. Barberis ${ }^{50 a, 50 b}$, M. Barbero ${ }^{21}$, D.Y. Bardin ${ }^{64}$, T. Barillari ${ }^{99}$, M. Barisonzi ${ }^{175}$, T. Barklow ${ }^{143}$, N. Barlow ${ }^{28}$, B.M. Barnett ${ }^{129}$, R.M. Barnett ${ }^{15}$, A. Baroncelli ${ }^{134 a}$, G. Barone ${ }^{49}$, A.J. Barr ${ }^{118}$, F. Barreiro ${ }^{80}$,

J. Barreiro Guimarães da Costa ${ }^{57}$, P. Barrillon ${ }^{115}$, R. Bartoldus ${ }^{143}$, A.E. Barton ${ }^{71}$, V. Bartsch ${ }^{149}$, A. Basye ${ }^{165}$, R.L. Bates ${ }^{53}$, L. Batkova ${ }^{144 a}$, J.R. Batley ${ }^{28}$, A. Battaglia ${ }^{17}$, M. Battistin ${ }^{30}$, F. Bauer ${ }^{136}$, H.S. Bawa ${ }^{143, g}$, S. Beale ${ }^{98}$, T. Beau ${ }^{78}$, P.H. Beauchemin ${ }^{161}$, R. Beccherle ${ }^{50 a}$, P. Bechtle ${ }^{21}$, H.P. Beck ${ }^{17}$, K. Becker ${ }^{175}$, S. Becker ${ }^{98}$, M. Beckingham ${ }^{138}$, K.H. Becks ${ }^{175}$, A.J. Beddall ${ }^{19 c}$, A. Beddall ${ }^{19 c}$, S. Bedikian ${ }^{176}$, V.A. Bednyakov ${ }^{64}$, C.P. Bee ${ }^{83}$, L.J. Beemster ${ }^{105}$, M. Begel ${ }^{25}$, S. Behar Harpaz ${ }^{152}$, P.K. Behera ${ }^{62}$, M. Beimforde ${ }^{99}$, C. Belanger-Champagne ${ }^{85}$, P.J. Bell ${ }^{49}$, W.H. Bell ${ }^{49}$, G. Bella ${ }^{153}$, L. Bellagamba ${ }^{20 a}$, M. Bellomo ${ }^{30}$, A. Belloni ${ }^{57}$, O. Beloborodova ${ }^{107, h}$, K. Belotskiy ${ }^{96}$, O. Beltramello ${ }^{30}$, O. Benary ${ }^{153}$, D. Benchekroun ${ }^{135 a}$, K. Bendtz ${ }^{146 a, 146 b}$, N. Benekos ${ }^{165}$, Y. Benhammou ${ }^{153}$,

E. Benhar Noccioli ${ }^{49}$, J.A. Benitez Garcia ${ }^{159 b}$, D.P. Benjamin ${ }^{45}$, M. Benoit ${ }^{115}$, J.R. Bensinger ${ }^{23}$, K. Benslama ${ }^{130}$, S. Bentvelsen ${ }^{105}$, D. Berge ${ }^{30}$, E. Bergeaas Kuutmann ${ }^{42}$, N. Berger ${ }^{5}$, F. Berghaus ${ }^{169}$, E. Berglund ${ }^{105}$, J. Beringer ${ }^{15}$, P. Bernat ${ }^{77}$, R. Bernhard ${ }^{48}$, C. Bernius ${ }^{25}$, T. Berry ${ }^{76}$, C. Bertella ${ }^{83}$, A. Bertin 20a,20b, F. Bertolucci'122a,122b ${ }^{2}$, M.I. Besana ${ }^{89 a, 89 b}$, G.J. Besjes ${ }^{104}$, N. Besson ${ }^{136}$, S. Bethke ${ }^{99}$, W. Bhimji ${ }^{46}$, R.M. Bianchi ${ }^{30}$, L. Bianchini ${ }^{23}$, M. Bianco ${ }^{72 a, 72 b}$, O. Biebel ${ }^{98}$, S.P. Bieniek ${ }^{77}$, K. Bierwagen ${ }^{54}$, J. Biesiada ${ }^{15}$, M. Biglietti ${ }^{134 a}$, H. Bilokon ${ }^{47}$, M. Bindi ${ }^{20 a, 20 b}$, S. Binet $^{115}$, A. Bingul ${ }^{19 c}$, C. Bini ${ }^{132 a, 132 b}$, C. Biscarat ${ }^{178}$, B. Bittner ${ }^{99}$, K.M. Black ${ }^{22}$, R.E. Blair ${ }^{6}$, J.-B. Blanchard ${ }^{136}$, G. Blanchot ${ }^{30}$, T. Blazek ${ }^{144 a}$, I. Bloch ${ }^{42}$, C. Blocker ${ }^{23}$, J. Blocki ${ }^{39}$, A. Blondel ${ }^{49}$, W. Blum ${ }^{81}$, U. Blumenschein ${ }^{54}$, G.J. Bobbink ${ }^{105}$, V.S. Bobrovnikov ${ }^{107}$, S.S. Bocchetta ${ }^{79}$, A. Bocci ${ }^{45}$, C.R. Boddy ${ }^{118}$, M. Boehler ${ }^{48}$, J. Boek ${ }^{175}$, T.T. Boek ${ }^{175}$, N. Boelaert ${ }^{36}$, J.A. Bogaerts ${ }^{30}$, A. Bogdanchikov ${ }^{107}$, A. Bogouch ${ }^{90, *}$, C. Bohm ${ }^{146 a}$, J. Bohm $^{125}$, V. Boisvert ${ }^{76}$, T. Bold ${ }^{38}$, V. Boldea ${ }^{26 a}$, N.M. Bolnet ${ }^{136}$, M. Bomben ${ }^{78}$, 
M. Bona ${ }^{75}$, M. Boonekamp ${ }^{136}$, S. Bordoni ${ }^{78}$, C. Borer ${ }^{17}$, A. Borisov ${ }^{128}$, G. Borissov ${ }^{71}$, I. Borjanovic ${ }^{13 a}$, M. Borri ${ }^{82}$, S. Borroni ${ }^{87}$, J. Bortfeldt ${ }^{98}$, V. Bortolotto ${ }^{134 a, 134 b}$, K. Bos ${ }^{105}$, D. Boscherini ${ }^{20 a}$, M. Bosman ${ }^{12}$, H. Boterenbrood ${ }^{105}$, J. Bouchami ${ }^{93}$, J. Boudreau ${ }^{123}$, E.V. Bouhova-Thacker ${ }^{71}$, D. Boumediene ${ }^{34}$, C. Bourdarios ${ }^{115}$, N. Bousson ${ }^{83}$, A. Boveia ${ }^{31}$, J. Boyd ${ }^{30}$, I.R. Boyko ${ }^{64}$, I. Bozovic-Jelisavcic ${ }^{13 b}$, J. Bracinik ${ }^{18}$, P. Branchini ${ }^{134 a}$, A. Brandt ${ }^{8}$, G. Brandt ${ }^{118}$, O. Brandt ${ }^{54}$, U. Bratzler ${ }^{156}$, B. Brau ${ }^{84}$, J.E. Brau ${ }^{114}$, H.M. Braun ${ }^{175, *}$, S.F. Brazzale ${ }^{164 a, 164 c}$, B. Brelier ${ }^{158}$, J. Bremer ${ }^{30}$, K. Brendlinger ${ }^{120}$, R. Brenner ${ }^{166}$, S. Bressler ${ }^{172}$, D. Britton ${ }^{53}$, F.M. Brochu ${ }^{28}$, I. Brock ${ }^{21}$, R. Brock ${ }^{88}$, F. Broggi ${ }^{89 a}$, C. Bromberg ${ }^{88}$, J. Bronner ${ }^{99}$, G. Brooijmans ${ }^{35}$, T. Brooks ${ }^{76}$, W.K. Brooks ${ }^{32 b}$, G. Brown ${ }^{82}$, H. Brown $^{8}$, R. Bruce ${ }^{30}$, P.A. Bruckman de Renstrom ${ }^{39}$, D. Bruncko ${ }^{144 b}$, R. Bruneliere ${ }^{48}$, S. Brunet $^{60}$, A. Bruni $^{20 a}$, G. Bruni ${ }^{20 a}$, M. Bruschi ${ }^{20 a}$, T. Buanes ${ }^{14}$, Q. Buat ${ }^{55}$, F. Bucci ${ }^{49}$, J. Buchanan ${ }^{118}$, P. Buchholz ${ }^{141}$, R.M. Buckingham ${ }^{118}$, A.G. Buckley ${ }^{46}$, S.I. Buda ${ }^{26 a}$, I.A. Budagov ${ }^{64}$, B. Budick ${ }^{108}$, V. Büscher ${ }^{81}$, L. Bugge ${ }^{117}$, O. Bulekov 96 , A.C. Bundock ${ }^{73}$, M. Bunse ${ }^{43}$, T. Buran ${ }^{117}$, H. Burckhart ${ }^{30}$, S. Burdin ${ }^{73}$, T. Burgess ${ }^{14}$, S. Burke ${ }^{129}$, E. Busato ${ }^{34}$, P. Bussey ${ }^{53}$, C.P. Buszello ${ }^{166}$, B. Butler ${ }^{143}$, J.M. Butler ${ }^{22}$, C.M. Buttar ${ }^{53}$, J.M. Butterworth ${ }^{77}$, W. Buttinger ${ }^{28}$, M. Byszewski ${ }^{30}$, S. Cabrera Urbán ${ }^{167}$, D. Caforio ${ }^{20 a}, 20 b$, O. Cakir ${ }^{4 a}$, P. Calafiura ${ }^{15}$, G. Calderini ${ }^{78}$, P. Calfayan ${ }^{98}$, R. Calkins ${ }^{106}$, L.P. Caloba ${ }^{24 a}$, R. Caloi ${ }^{132 a, 132 b}$, D. Calvet ${ }^{34}$, S. Calvet ${ }^{34}$, R. Camacho Toro ${ }^{34}$, P. Camarri133a,133b, D. Cameron ${ }^{117}$, L.M. Caminada ${ }^{15}$, R. Caminal Armadans ${ }^{12}$, S. Campana ${ }^{30}$, M. Campanelli ${ }^{77}$, V. Canale ${ }^{102 a, 102 b}$, F. Canelli ${ }^{31}$, A. Canepa ${ }^{159 a}$, J. Cantero ${ }^{80}$, R. Cantrill ${ }^{76}$, L. Capasso ${ }^{102 a, 102 b}$, M.D.M. Capeans Garrido ${ }^{30}$, I. Caprini ${ }^{26 a}$, M. Caprini ${ }^{26 a}$, D. Capriotti ${ }^{99}$, M. Capua ${ }^{37 a, 37 b}$, R. Caputo ${ }^{81}$, R. Cardarelli ${ }^{133 a}$, T. Carli $^{30}$, G. Carlino ${ }^{102 a}$, L. Carminati ${ }^{89 a, 89 b}$, B. Caron ${ }^{85}$, S. Caron ${ }^{104}$, E. Carquin ${ }^{32 b}$, G.D. Carrillo-Montoya ${ }^{145 b}$, A.A. Carter $^{75}$, J.R. Carter $^{28}$, J. Carvalho ${ }^{124 a, i}$, D. Casadei ${ }^{108}$, M.P. Casado ${ }^{12}$, M. Cascella ${ }^{122 a, 122 b}$, C. Caso $^{50 a, 50 b, *}$, A.M. Castaneda Hernandez ${ }^{173, j}$, E. Castaneda-Miranda ${ }^{173}$, V. Castillo Gimenez ${ }^{167}$, N.F. Castro ${ }^{124 a}$, G. Cataldi ${ }^{72 a}$, P. Catastini ${ }^{57}$, A. Catinaccio ${ }^{30}$, J.R. Catmore ${ }^{30}$, A. Cattai ${ }^{30}$, G. Cattani ${ }^{133 a, 133 b}$, S. Caughron ${ }^{88}$, V. Cavaliere ${ }^{165}$, P. Cavalleri ${ }^{78}$, D. Cavalli ${ }^{89 a}$, M. Cavalli-Sforza ${ }^{12}$, V. Cavasinni ${ }^{122 a, 122 b}$, F. Ceradini ${ }^{134 a, 134 b}$, A.S. Cerqueira ${ }^{24 b}$, A. Cerri ${ }^{30}$, L. Cerrito ${ }^{75}$, F. Cerutti ${ }^{47}$, S.A. Cetin ${ }^{19 b}$, A. Chafaq ${ }^{135 a}$, D. Chakraborty ${ }^{106}$, I. Chalupkova ${ }^{127}$, K. Chan ${ }^{3}$, P. Chang ${ }^{165}$, B. Chapleau ${ }^{85}$, J.D. Chapman ${ }^{28}$, J.W. Chapman ${ }^{87}$, E. Chareyre ${ }^{78}$, D.G. Charlton ${ }^{18}$, V. Chavda ${ }^{82}$, C.A. Chavez Barajas ${ }^{30}$, S. Cheatham ${ }^{85}$, S. Chekanov ${ }^{6}$, S.V. Chekulaev ${ }^{159 a}$, G.A. Chelkov ${ }^{64}$, M.A. Chelstowska ${ }^{104}$, C. Chen ${ }^{63}$, H. Chen ${ }^{25}$, S. Chen ${ }^{33 c}$, X. Chen ${ }^{173}$, Y. Chen ${ }^{35}$, Y. Cheng ${ }^{31}$, A. Cheplakov ${ }^{64}$, R. Cherkaoui El Moursli ${ }^{135 e}$, V. Chernyatin ${ }^{25}$, E. Cheu ${ }^{7}$, S.L. Cheung ${ }^{158}$, L. Chevalier ${ }^{136}$, G. Chiefari ${ }^{102 a, 102 b}$, L. Chikovani ${ }^{51 a, *}$, J.T. Childers ${ }^{30}$, A. Chilingarov ${ }^{71}$, G. Chiodini ${ }^{72 a}$, A.S. Chisholm ${ }^{18}$, R.T. Chislett ${ }^{77}$, A. Chitan $^{26 a}$, M.V. Chizhov ${ }^{64}$, G. Choudalakis ${ }^{31}$, S. Chouridou ${ }^{137}$, I.A. Christidi ${ }^{77}$, A. Christov ${ }^{48}$,

D. Chromek-Burckhart ${ }^{30}$, M.L. Chu ${ }^{151}$, J. Chudoba ${ }^{125}$, G. Ciapetti132a,132b, A.K. Ciftci ${ }^{4 a}$, R. Ciftci ${ }^{4 a}$,

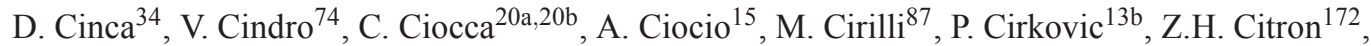
M. Citterio ${ }^{89 a}$, M. Ciubancan ${ }^{26 a}$, A. Clark ${ }^{49}$, P.J. Clark ${ }^{46}$, R.N. Clarke ${ }^{15}$, W. Cleland ${ }^{123}$, J.C. Clemens ${ }^{83}$,

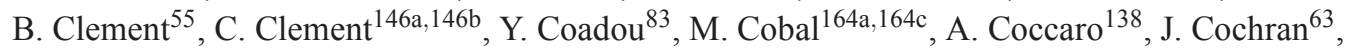
L. Coffey ${ }^{23}$, J.G. Cogan ${ }^{143}$, J. Coggeshall ${ }^{165}$, E. Cogneras ${ }^{178}$, J. Colas ${ }^{5}$, S. Cole ${ }^{106}$, A.P. Colijn ${ }^{105}$, N.J. Collins ${ }^{18}$, C. Collins-Tooth ${ }^{53}$, J. Collot ${ }^{55}$, T. Colombo ${ }^{119 a, 119 b}$, G. Colon ${ }^{84}$, G. Compostella ${ }^{99}$, P. Conde Muiño ${ }^{124 a}$, E. Coniavitis ${ }^{166}$, M.C. Conidi ${ }^{12}$, S.M. Consonni ${ }^{89 a}$ a 89 b , V. Consorti ${ }^{48}$,

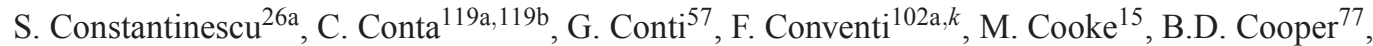
A.M. Cooper-Sarkar ${ }^{118}$, K. Copic ${ }^{15}$, T. Cornelissen ${ }^{175}$, M. Corradi ${ }^{20 a}$, F. Corriveau ${ }^{85, l}$,

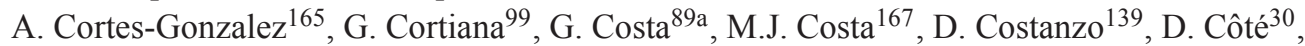
L. Courneyea ${ }^{169}$, G. Cowan $^{76}$, C. Cowden ${ }^{28}$, B.E. Cox ${ }^{82}$, K. Cranmer ${ }^{108}$, F. Crescioli ${ }^{78}$, M. Cristinziani ${ }^{21}$, G. Crosetti ${ }^{37 a, 37 b}$, S. Crépé-Renaudin ${ }^{55}$, C.-M. Cuciuc ${ }^{26 a}$, C. Cuenca Almenar ${ }^{176}$,

T. Cuhadar Donszelmann ${ }^{139}$, J. Cummings ${ }^{176}$, M. Curatolo ${ }^{47}$, C.J. Curtis ${ }^{18}$, C. Cuthbert ${ }^{150}$, P. Cwetanski ${ }^{60}$, H. Czirr ${ }^{141}$, P. Czodrowski ${ }^{44}$, Z. Czyczula ${ }^{176}$, S. D’Auria ${ }^{53}$, M. D’Onofrio ${ }^{73}$, A. D’Orazio ${ }^{132 a, 132 b}$, M.J. Da Cunha Sargedas De Sousa ${ }^{124 a}$, C. Da Via ${ }^{82}$, W. Dabrowski ${ }^{38}$, A. Dafinca ${ }^{118}$, T. Dai ${ }^{87}$,

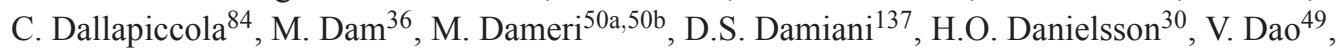


G. Darbo ${ }^{50 a}$, G.L. Darlea ${ }^{26 b}$, J.A. Dassoulas ${ }^{42}$, W. Davey ${ }^{21}$, T. Davidek ${ }^{127}$, N. Davidson ${ }^{86}$, R. Davidson ${ }^{71}$, E. Davies ${ }^{118, d}$, M. Davies ${ }^{93}$, O. Davignon ${ }^{78}$, A.R. Davison ${ }^{77}$, Y. Davygora ${ }^{58 a}$, E. Dawe ${ }^{142}$, I. Dawson ${ }^{139}$, R.K. Daya-Ishmukhametova ${ }^{23}$, K. De ${ }^{8}$, R. de Asmundis ${ }^{102 a}$, S. De Castro ${ }^{20 a, 20 b}$, S. De Cecco ${ }^{78}$, J. de Graat ${ }^{98}$, N. De Groot ${ }^{104}$, P. de Jong ${ }^{105}$, C. De La Taille ${ }^{115}$, H. De la Torre ${ }^{80}$, F. De Lorenzi ${ }^{63}$, L. de Mora ${ }^{71}$, L. De Nooij ${ }^{105}$, D. De Pedis ${ }^{132 a}$, A. De Salvo ${ }^{132 a}$, U. De Sanctis ${ }^{164 a, 164 c}$, A. De Santo ${ }^{149}$, J.B. De Vivie De Regie ${ }^{115}$, G. De Zorzi ${ }^{132 a, 132 b}$, W.J. Dearnaley ${ }^{71}$, R. Debbe ${ }^{25}$, C. Debenedetti ${ }^{46}$, B. Dechenaux ${ }^{55}$, D.V. Dedovich ${ }^{64}$, J. Degenhardt ${ }^{120}$, J. Del Peso ${ }^{80}$, T. Del Prete ${ }^{122 a, 122 b}$, T. Delemontex ${ }^{55}$, M. Deliyergiyev ${ }^{74}$, A. Dell'Acqua ${ }^{30}$, L. Dell'Asta ${ }^{22}$, M. Della Pietra ${ }^{102 a, k}$, D. della Volpe ${ }^{102 a, 102 b}$, M. Delmastro ${ }^{5}$, P.A. Delsart ${ }^{55}$, C. Deluca ${ }^{105}$, S. Demers ${ }^{176}$, M. Demichev ${ }^{64}$, B. Demirkoz ${ }^{12, m}$,

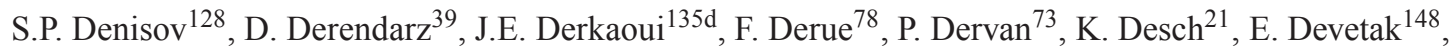
P.O. Deviveiros ${ }^{105}$, A. Dewhurst ${ }^{129}$, B. DeWilde ${ }^{148}$, S. Dhaliwal $^{158}$, R. Dhullipudi $^{25, n}$,

A. Di Ciaccio ${ }^{133 a, 133 b}$, L. Di Ciaccio ${ }^{5}$, C. Di Donato ${ }^{102 a, 102 b}$, A. Di Girolamo ${ }^{30}$, B. Di Girolamo ${ }^{30}$, S. Di Luise ${ }^{134 a, 134 b}$, A. Di Mattia ${ }^{173}$, B. Di Micco ${ }^{30}$, R. Di Nardo ${ }^{47}$, A. Di Simone ${ }^{133 a, 133 b}$, R. Di Sipio 20a,20b, M.A. Diaz ${ }^{32 a}$, E.B. Diehl ${ }^{87}$, J. Dietrich ${ }^{42}$, T.A. Dietzsch ${ }^{58 a}$, S. Diglio ${ }^{86}$,

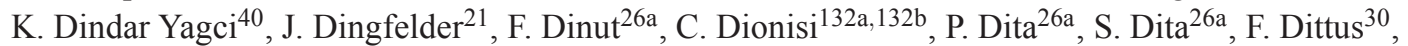
F. Djama ${ }^{83}$, T. Djobava ${ }^{51 b}$, M.A.B. do Vale ${ }^{24 c}$, A. Do Valle Wemans ${ }^{124 a, o}$, T.K.O. Doan ${ }^{5}$, M. Dobbs ${ }^{85}$,

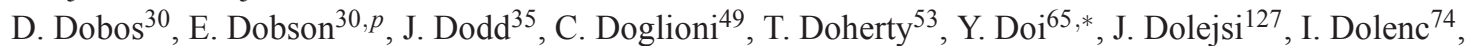
Z. Dolezal ${ }^{127}$, B.A. Dolgoshein ${ }^{96, *}$, T. Dohmae ${ }^{155}$, M. Donadelli24d ${ }^{24}$ J. Donini ${ }^{34}$, J. Dopke $^{30}$, A. Doria $^{102 a}$,

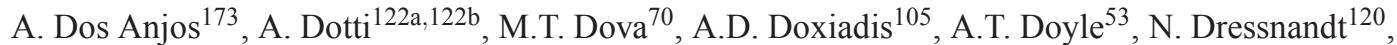
M. Dris ${ }^{10}$, J. Dubbert ${ }^{99}$, S. Dube ${ }^{15}$, E. Duchovni ${ }^{172}$, G. Duckeck ${ }^{98}$, D. Duda ${ }^{175}$, A. Dudarev ${ }^{30}$,

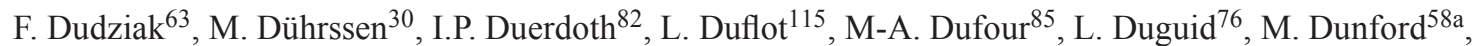
H. Duran Yildiz ${ }^{4 a}$, R. Duxfield ${ }^{139}$, M. Dwuznik ${ }^{38}$, F. Dydak ${ }^{30}$, M. Düren ${ }^{52}$, W.L. Ebenstein ${ }^{45}$, J. Ebke ${ }^{98}$, S. Eckweiler ${ }^{81}$, K. Edmonds ${ }^{81}$, W. Edson ${ }^{2}$, C.A. Edwards ${ }^{76}$, N.C. Edwards ${ }^{53}$, W. Ehrenfeld ${ }^{42}$, T. Eifert ${ }^{143}$, G. Eigen ${ }^{14}$, K. Einsweiler ${ }^{15}$, E. Eisenhandler ${ }^{75}$, T. Ekelof ${ }^{166}$, M. El Kacimi ${ }^{135 c}$, M. Ellert ${ }^{166}$, S. Elles ${ }^{5}$, F. Ellinghaus ${ }^{81}$, K. Ellis ${ }^{75}$, N. Ellis ${ }^{30}$, J. Elmsheuser ${ }^{98}$, M. Elsing ${ }^{30}$, D. Emeliyanov ${ }^{129}$, R. Engelmann ${ }^{148}$, A. Engl $^{98}$, B. Epp ${ }^{61}$, J. Erdmann ${ }^{54}$, A. Ereditato ${ }^{17}$, D. Eriksson ${ }^{146 a}$, J. Ernst ${ }^{2}$, M. Ernst ${ }^{25}$, J. Ernwein ${ }^{136}$, D. Errede ${ }^{165}$, S. Errede ${ }^{165}$, E. Ertel ${ }^{81}$, M. Escalier ${ }^{115}$, H. Esch ${ }^{43}$, C. Escobar ${ }^{123}$, X. Espinal Curull ${ }^{12}$, B. Esposito ${ }^{47}$, F. Etienne ${ }^{83}$, A.I. Etienvre ${ }^{136}$, E. Etzion ${ }^{153}$, D. Evangelakou ${ }^{54}$, H. Evans ${ }^{60}$, L. Fabbri ${ }^{20 a, 20 b}$, C. Fabre $^{30}$, R.M. Fakhrutdinov ${ }^{128}$, S. Falciano ${ }^{132 a}$, Y. Fang ${ }^{33 a}$, M. Fanti ${ }^{89 a}$,89b , A. Farbin ${ }^{8}$, A. Farilla ${ }^{134 a}$, J. Farley ${ }^{148}$, T. Farooque ${ }^{158}$, S. Farrell ${ }^{163}$, S.M. Farrington ${ }^{170}$, P. Farthouat ${ }^{30}$, F. Fassi ${ }^{167}$, P. Fassnacht ${ }^{30}$, D. Fassouliotis ${ }^{9}$, B. Fatholahzadeh ${ }^{158}$, A. Favareto ${ }^{89 a, 89 b}$, L. Fayard ${ }^{115}$, S. Fazio ${ }^{37 a, 37 b}$, R. Febbraro ${ }^{34}$, P. Federic ${ }^{144}$, O.L. Fedin ${ }^{121}$, W. Fedorko ${ }^{88}$, M. Fehling-Kaschek ${ }^{48}$, L. Feligioni ${ }^{83}$, C. Feng ${ }^{33 d}$, E.J. Feng 6 , A.B. Fenyuk ${ }^{128}$, J. Ferencei ${ }^{144 b}$, W. Fernando ${ }^{6}$, S. Ferrag ${ }^{53}$, J. Ferrando ${ }^{53}$, V. Ferrara ${ }^{42}$, A. Ferrari ${ }^{166}$, P. Ferrari ${ }^{105}$, R. Ferrari ${ }^{119 a}$, D.E. Ferreira de Lima $^{53}$, A. Ferrer ${ }^{167}$, D. Ferrere ${ }^{49}$, C. Ferretti ${ }^{87}$, A. Ferretto Parodi ${ }^{50 a, 50 b}$, M. Fiascaris ${ }^{31}$, F. Fiedler ${ }^{81}$, A. Filipčič ${ }^{74}$, F. Filthaut $^{104}$, M. Fincke-Keeler ${ }^{169}$, M.C.N. Fiolhais ${ }^{124 a, i}$, L. Fiorini ${ }^{167}$, A. Firan ${ }^{40}$, G. Fischer ${ }^{42}$, M.J. Fisher ${ }^{109}$, M. Flechl ${ }^{48}$, I. Fleck ${ }^{141}$, J. Fleckner ${ }^{81}$, P. Fleischmann ${ }^{174}$, S. Fleischmann ${ }^{175}$, T. Flick ${ }^{175}$, A. Floderus ${ }^{79}$, L.R. Flores Castillo ${ }^{173}$, M.J. Flowerdew ${ }^{99}$, T. Fonseca Martin ${ }^{17}$, A. Formica ${ }^{136}$, A. Forti ${ }^{82}$, D. Fortin ${ }^{159 a}$, D. Fournier ${ }^{115}$, A.J. Fowler ${ }^{45}$, H. Fox ${ }^{71}$, P. Francavilla ${ }^{12}$, M. Franchini ${ }^{20 a, 20 b}$, S. Franchino119a,119b ${ }^{\text {, D. Francis }}{ }^{30}$, T. Frank ${ }^{172}$, M. Franklin ${ }^{57}$, S. Franz ${ }^{30}$, M. Fraternali ${ }^{119 a, 119 b}$, S. Fratina ${ }^{120}$, S.T. French ${ }^{28}$, C. Friedrich ${ }^{42}$, F. Friedrich ${ }^{44}$, R. Froeschl ${ }^{30}$, D. Froidevaux ${ }^{30}$, J.A. Frost ${ }^{28}$, C. Fukunaga ${ }^{156}$, E. Fullana Torregrosa ${ }^{30}$, B.G. Fulsom ${ }^{143}$, J. Fuster ${ }^{167}$, C. Gabaldon ${ }^{30}$, O. Gabizon ${ }^{172}$, T. Gadfort ${ }^{25}$, S. Gadomski ${ }^{49}$, G. Gagliardi ${ }^{50 a, 50 b}$, P. Gagnon ${ }^{60}$, C. Galea ${ }^{98}$, B. Galhardo ${ }^{124 a}$, E.J. Gallas ${ }^{118}$, V. Gallo ${ }^{17}$, B.J. Gallop ${ }^{129}$, P. Gallus ${ }^{125}$, K.K. Gan ${ }^{109}$, Y.S. Gao ${ }^{143, g}$, A. Gaponenko ${ }^{15}$, F. Garberson ${ }^{176}$, M. Garcia-Sciveres ${ }^{15}$, C. García ${ }^{167}$, J.E. García Navarro ${ }^{167}$, R.W. Gardner ${ }^{31}$, N. Garelli ${ }^{30}$, H. Garitaonandia ${ }^{105}$, V. Garonne ${ }^{30}$, C. Gatti $^{47}$, G. Gaudio ${ }^{119 a}$, B. Gaur ${ }^{141}$, L. Gauthier ${ }^{136}$, P. Gauzzi ${ }^{132 a, 132 b}$, I.L. Gavrilenko ${ }^{94}$, C. Gay ${ }^{168}$, G. Gaycken ${ }^{21}$, E.N. Gazis ${ }^{10}$, P. Ge ${ }^{33 d}$, Z. Gecse ${ }^{168}$, C.N.P. Gee ${ }^{129}$, D.A.A. Geerts ${ }^{105}$, Ch. Geich-Gimbel ${ }^{21}$,

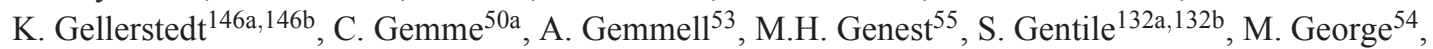


S. George ${ }^{76}$, P. Gerlach ${ }^{175}$, A. Gershon ${ }^{153}$, C. Geweniger ${ }^{58 a}$, H. Ghazlane ${ }^{135 b}$, N. Ghodbane ${ }^{34}$, B. Giacobbe ${ }^{20 a}$, S. Giagu ${ }^{132 a, 132 b}$, V. Giakoumopoulou ${ }^{9}$, V. Giangiobbe ${ }^{12}$, F. Gianotti ${ }^{30}$, B. Gibbard ${ }^{25}$, A. Gibson ${ }^{158}$, S.M. Gibson ${ }^{30}$, M. Gilchriese ${ }^{15}$, D. Gillberg ${ }^{29}$, A.R. Gillman ${ }^{129}$, D.M. Gingrich ${ }^{3, f}$, J. Ginzburg ${ }^{153}$, N. Giokaris ${ }^{9}$, M.P. Giordani ${ }^{164 c}$, R. Giordano ${ }^{102 a, 102 b}$, F.M. Giorgi ${ }^{16}$, P. Giovannini ${ }^{99}$, P.F. Giraud ${ }^{136}$, D. Giugni ${ }^{89 a}$, M. Giunta ${ }^{93}$, B.K. Gjelsten ${ }^{117}$, L.K. Gladilin ${ }^{97}$, C. Glasman ${ }^{80}$, J. Glatzer ${ }^{21}$, A. Glazov ${ }^{42}$, K.W. Glitza ${ }^{175}$, G.L. Glonti ${ }^{64}$, J.R. Goddard ${ }^{75}$, J. Godfrey ${ }^{142}$, J. Godlewski ${ }^{30}$, M. Goebel ${ }^{42}$, T. Göpfert ${ }^{44}$, C. Goeringer ${ }^{81}$, C. Gössling ${ }^{43}$, S. Goldfarb ${ }^{87}$, T. Golling ${ }^{176}$, A. Gomes ${ }^{124 a, c}$, L.S. Gomez Fajardo ${ }^{42}$, R. Gonçalo ${ }^{76}$, J. Goncalves Pinto Firmino Da Costa ${ }^{42}$, L. Gonella ${ }^{21}$, S. González de la $\mathrm{Hoz}^{167}$, G. Gonzalez Parra ${ }^{12}$, M.L. Gonzalez Silva ${ }^{27}$, S. Gonzalez-Sevilla ${ }^{49}$, J.J. Goodson ${ }^{148}$, L. Goossens ${ }^{30}$, P.A. Gorbounov ${ }^{95}$, H.A. Gordon ${ }^{25}$, I. Gorelov ${ }^{103}$, G. Gorfine ${ }^{175}$, B. Gorini ${ }^{30}$, E. Gorini ${ }^{72 a, 72 b}$, A. Gorišek ${ }^{74}$, E. Gornicki ${ }^{39}$, A.T. Goshaw ${ }^{6}$, M. Gosselink ${ }^{105}$, M.I. Gostkin ${ }^{64}$, I. Gough Eschrich ${ }^{163}$, M. Gouighri ${ }^{135 a}$, D. Goujdami ${ }^{135 c}$, M.P. Goulette ${ }^{49}$, A.G. Goussiou ${ }^{138}$, C. Goy ${ }^{5}$,

S. Gozpinar ${ }^{23}$, I. Grabowska-Bold ${ }^{38}$, P. Grafström ${ }^{20 a, 20 b}$, K-J. Grahn ${ }^{42}$, E. Gramstad ${ }^{117}$, F. Grancagnolo ${ }^{72 a}$, S. Grancagnolo ${ }^{16}$, V. Grassi ${ }^{148}$, V. Gratchev ${ }^{121}$, N. Grau ${ }^{35}$, H.M. Gray ${ }^{30}$, J.A. Gray ${ }^{148}$, E. Graziani ${ }^{134 a}$, O.G. Grebenyuk ${ }^{121}$, T. Greenshaw ${ }^{73}$, Z.D. Greenwood ${ }^{25, n}$, K. Gregersen ${ }^{36}$, I.M. Gregor ${ }^{42}$, P. Grenier ${ }^{143}$, J. Griffiths ${ }^{8}$, N. Grigalashvili ${ }^{64}$, A.A. Grillo ${ }^{137}$, S. Grinstein ${ }^{12}$, Ph. Gris ${ }^{34}$, Y.V. Grishkevich ${ }^{97}$, J.-F. Grivaz ${ }^{115}$, E. Gross ${ }^{172}$, J. Grosse-Knetter ${ }^{54}$, J. Groth-Jensen ${ }^{172}$, K. Grybel ${ }^{141}$, D. Guest ${ }^{176}$, C. Guicheney ${ }^{34}$, E. Guido ${ }^{50 \mathrm{a}, 50 \mathrm{~b}}, \mathrm{~S} . \mathrm{Guindon}^{54}$, U. Gul ${ }^{53}$, J. Gunther ${ }^{125}$, B. Guo ${ }^{158}$, J. Guo ${ }^{35}$,

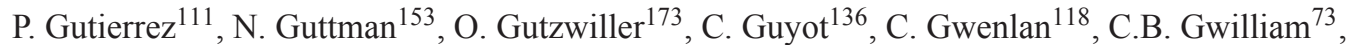

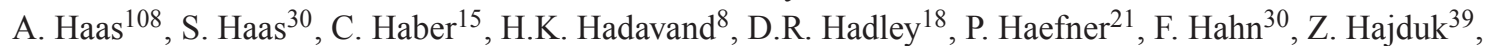

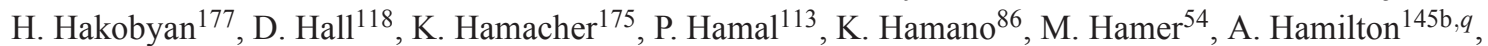

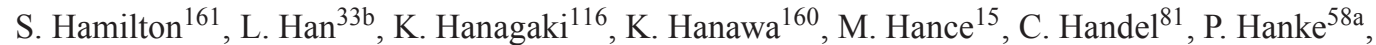

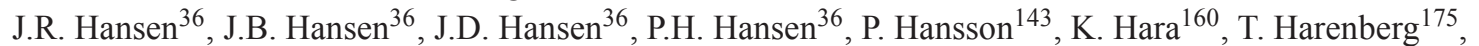
S. Harkusha ${ }^{90}$, D. Harper ${ }^{87}$, R.D. Harrington ${ }^{46}$, O.M. Harris ${ }^{138}$, J. Hartert ${ }^{48}$, F. Hartjes ${ }^{105}$, T. Haruyama ${ }^{65}$, A. Harvey ${ }^{56}$, S. Hasegawa ${ }^{101}$, Y. Hasegawa ${ }^{140}$, S. Hassani ${ }^{136}$, S. Haug ${ }^{17}$, M. Hauschild ${ }^{30}$, R. Hauser ${ }^{88}$, M. Havranek ${ }^{21}$, C.M. Hawkes ${ }^{18}$, R.J. Hawkings ${ }^{30}$, A.D. Hawkins ${ }^{79}$, T. Hayakawa ${ }^{66}$, T. Hayashi ${ }^{160}$,

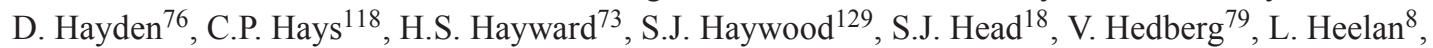
S. Heim ${ }^{120}$, B. Heinemann ${ }^{15}$, S. Heisterkamp ${ }^{36}$, L. Helary ${ }^{22}$, C. Heller ${ }^{98}$, M. Heller ${ }^{30}$, S. Hellman ${ }^{146 a, 146 b}$, D. Hellmich ${ }^{21}$, C. Helsens ${ }^{12}$, R.C.W. Henderson ${ }^{71}$, M. Henke ${ }^{58 a}$, A. Henrichs ${ }^{176}$,

A.M. Henriques Correia ${ }^{30}$, S. Henrot-Versille ${ }^{115}$, C. Hensel $^{54}$, T. Hen $\beta^{175}$, C.M. Hernandez ${ }^{8}$, Y. Hernández Jiménez ${ }^{167}$, R. Herrberg ${ }^{16}$, G. Herten ${ }^{48}$, R. Hertenberger ${ }^{98}$, L. Hervas ${ }^{30}$, G.G. Hesketh ${ }^{77}$, N.P. Hessey ${ }^{105}$, E. Higón-Rodriguez ${ }^{167}$, J.C. Hill ${ }^{28}$, K.H. Hiller ${ }^{42}$, S. Hillert ${ }^{21}$, S.J. Hillier ${ }^{18}$,

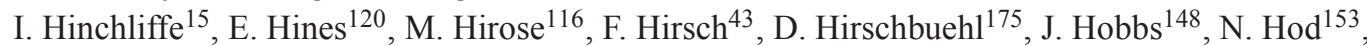
M.C. Hodgkinson ${ }^{139}$, P. Hodgson ${ }^{139}$, A. Hoecker ${ }^{30}$, M.R. Hoeferkamp ${ }^{103}$, J. Hoffman ${ }^{40}$, D. Hoffmann ${ }^{83}$, M. Hohlfeld ${ }^{81}$, M. Holder ${ }^{141}$, S.O. Holmgren ${ }^{146 a}$, T. Holy ${ }^{126}$, J.L. Holzbauer ${ }^{88}$, T.M. Hong ${ }^{120}$, L. Hooft van Huysduynen ${ }^{108}$, S. Horner ${ }^{48}$, J-Y. Hostachy ${ }^{55}$, S. Hou ${ }^{151}$, A. Hoummada ${ }^{135 a}$, J. Howard ${ }^{118}$, J. Howarth ${ }^{82}$, I. Hristova ${ }^{16}$, J. Hrivnac ${ }^{115}$, T. Hryn'ova ${ }^{5}$, P.J. Hsu ${ }^{81}$, S.-C. Hsu ${ }^{15}$, D. Hu ${ }^{35}$, Z. Hubacek ${ }^{126}$, F. Hubaut ${ }^{83}$, F. Huegging ${ }^{21}$, A. Huettmann ${ }^{42}$, T.B. Huffman ${ }^{118}$, E.W. Hughes ${ }^{35}$, G. Hughes ${ }^{71}$,

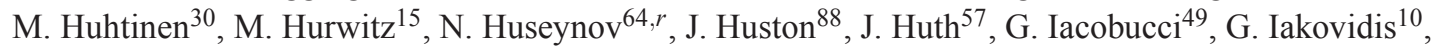
M. Ibbotson ${ }^{82}$, I. Ibragimov ${ }^{141}$, L. Iconomidou-Fayard ${ }^{115}$, J. Idarraga $^{115}$, P. Iengo $^{102 a}$, O. Igonkina ${ }^{105}$, Y. Ikegami ${ }^{65}$, M. Ikeno ${ }^{65}$, D. Iliadis ${ }^{154}$, N. Ilic ${ }^{158}$, T. Ince ${ }^{99}$, J. Inigo-Golfin ${ }^{30}$, P. Ioannou ${ }^{9}$, M. Iodice ${ }^{134 a}$, K. Iordanidou 9 , V. Ippolito ${ }^{132 a, 132 b}$, A. Irles Quiles ${ }^{167}$, C. Isaksson ${ }^{166}$, M. Ishino ${ }^{67}$, M. Ishitsuka ${ }^{157}$, R. Ishmukhametov ${ }^{109}$, C. Issever ${ }^{118}$, S. Istin ${ }^{19 a}$, A.V. Ivashin ${ }^{128}$, W. Iwanski ${ }^{39}$, H. Iwasaki ${ }^{65}$, J.M. Izen $^{41}$, V. Izzo ${ }^{102 a}$, B. Jackson ${ }^{120}$, J.N. Jackson ${ }^{73}$, P. Jackson ${ }^{1}$, M.R. Jaekel ${ }^{30}$, V. Jain ${ }^{60}$, K. Jakobs ${ }^{48}$, S. Jakobsen ${ }^{36}$, T. Jakoubek ${ }^{125}$, J. Jakubek ${ }^{126}$, D.O. Jamin ${ }^{151}$, D.K. Jana ${ }^{111}$, E. Jansen ${ }^{77}$, H. Jansen ${ }^{30}$, J. Janssen ${ }^{21}$, A. Jantsch ${ }^{99}$, M. Janus ${ }^{48}$, R.C. Jared ${ }^{173}$, G. Jarlskog ${ }^{79}$, L. Jeanty ${ }^{57}$, I. Jen-La Plante ${ }^{31}$, D. Jennens ${ }^{86}$, P. Jenni ${ }^{30}$, A.E. Loevschall-Jensen ${ }^{36}$, P. Jež ${ }^{36}$, S. Jézéquel ${ }^{5}$, M.K. Jha ${ }^{20 a}$, H. Ji ${ }^{173}$, W. Ji ${ }^{81}$, J. Jia ${ }^{148}$, Y. Jiang ${ }^{33 b}$, M. Jimenez Belenguer ${ }^{42}$, S. Jin ${ }^{33 a}$, O. Jinnouchi ${ }^{157}$, M.D. Joergensen ${ }^{36}$, D. Joffe ${ }^{40}$, 
M. Johansen ${ }^{146 a, 146 b}$, K.E. Johansson ${ }^{146 a}$, P. Johansson ${ }^{139}$, S. Johnert ${ }^{42}$, K.A. Johns ${ }^{7}$, K. Jon-And ${ }^{146 a, 146 b}$, G. Jones ${ }^{170}$, R.W.L. Jones ${ }^{71}$, T.J. Jones ${ }^{73}$, C. Joram ${ }^{30}$, P.M. Jorge ${ }^{124 a}$, K.D. Joshi ${ }^{82}$, J. Jovicevic ${ }^{147}$, T. Jovin ${ }^{13 b}$, X. Ju ${ }^{173}$, C.A. Jung ${ }^{43}$, R.M. Jungst ${ }^{30}$, V. Juranek ${ }^{125}$, P. Jussel ${ }^{61}$, A. Juste Rozas ${ }^{12}$, S. Kabana ${ }^{17}$, M. Kaci ${ }^{167}$, A. Kaczmarska ${ }^{39}$, P. Kadlecik ${ }^{36}$, M. Kado ${ }^{115}$, H. Kagan ${ }^{109}$, M. Kagan ${ }^{57}$, E. Kajomovitz ${ }^{152}$, S. Kalinin ${ }^{175}$, L.V. Kalinovskaya ${ }^{64}$, S. Kama ${ }^{40}$, N. Kanaya ${ }^{155}$, M. Kaneda ${ }^{30}$, S. Kaneti ${ }^{28}$, T. Kanno ${ }^{157}$, V.A. Kantserov ${ }^{96}$, J. Kanzaki ${ }^{65}$, B. Kaplan ${ }^{108}$, A. Kapliy ${ }^{31}$, J. Kaplon ${ }^{30}$, D. Kar ${ }^{53}$, M. Karagounis ${ }^{21}$, K. Karakostas ${ }^{10}$, M. Karnevskiy ${ }^{42}$, V. Kartvelishvili ${ }^{71}$, A.N. Karyukhin ${ }^{128}$, L. Kashif ${ }^{173}$, G. Kasieczka ${ }^{58 b}$, R.D. Kass ${ }^{109}$, A. Kastanas ${ }^{14}$, M. Kataoka ${ }^{5}$, Y. Kataoka ${ }^{155}$, E. Katsoufis ${ }^{10}$, J. Katzy ${ }^{42}$, V. Kaushik ${ }^{7}$, K. Kawagoe ${ }^{69}$, T. Kawamoto ${ }^{155}$, G. Kawamura ${ }^{81}$, M.S. Kayl ${ }^{105}$, S. Kazama ${ }^{155}$, V.F. Kazanin ${ }^{107}$, M.Y. Kazarinov ${ }^{64}$, R. Keeler ${ }^{169}$, P.T. Keener ${ }^{120}$, R. Kehoe ${ }^{40}$, M. Keil ${ }^{54}$, G.D. Kekelidze ${ }^{64}$, J.S. Keller ${ }^{138}$, M. Kenyon ${ }^{53}$, O. Kepka ${ }^{125}$, N. Kerschen ${ }^{30}$, B.P. Kerševan ${ }^{74}$, S. Kersten ${ }^{175}$, K. Kessoku ${ }^{155}$, J. Keung ${ }^{158}$, F. Khalil-zada ${ }^{11}$, H. Khandanyan ${ }^{146 a, 146 b}$, A. Khanov ${ }^{112}$, D. Kharchenko ${ }^{64}$, A. Khodinov ${ }^{96}$, A. Khomich ${ }^{58 a}$, T.J. Khoo ${ }^{28}$, G. Khoriauli ${ }^{21}$, A. Khoroshilov ${ }^{175}$, V. Khovanskiy ${ }^{95}$, E. Khramov ${ }^{64}$, J. Khubua ${ }^{51 b}$, H. Kim ${ }^{146 a}, 146 b$, S.H. Kim ${ }^{160}$, N. Kimura ${ }^{171}$, O. Kind ${ }^{16}$, B.T. King ${ }^{73}$, M. King ${ }^{66}$, R.S.B. King ${ }^{18}$, J. Kirk ${ }^{129}$, A.E. Kiryunin ${ }^{99}$, T. Kishimoto ${ }^{66}$, D. Kisielewska ${ }^{38}$, T. Kitamura ${ }^{66}$, T. Kittelmann ${ }^{123}$, K. Kiuchi ${ }^{160}$, E. Kladiva ${ }^{144 b}$, M. Klein ${ }^{73}$, U. Klein ${ }^{73}$, K. Kleinknecht ${ }^{81}$, M. Klemetti ${ }^{85}$, A. Klier ${ }^{172}$, P. Klimek ${ }^{146 a, 146 b}$, A. Klimentov ${ }^{25}$, R. Klingenberg ${ }^{43}$, J.A. Klinger ${ }^{82}$, E.B. Klinkby ${ }^{36}$, T. Klioutchnikova ${ }^{30}$, P.F. Klok ${ }^{104}$, S. Klous ${ }^{105}$, E.-E. Kluge ${ }^{58 a}$, T. Kluge ${ }^{73}$, P. Kluit ${ }^{105}$, S. Kluth ${ }^{99}$, E. Kneringer ${ }^{61}$, E.B.F.G. Knoops ${ }^{83}$, A. Knue ${ }^{54}$, B.R. Ko ${ }^{45}$, T. Kobayashi ${ }^{155}$, M. Kobel ${ }^{44}$, M. Kocian ${ }^{143}$, P. Kodys ${ }^{127}$, K. Köneke ${ }^{30}$, A.C. König ${ }^{104}$, S. Koenig ${ }^{81}$, L. Köpke ${ }^{81}$, F. Koetsveld ${ }^{104}$, P. Koevesarki ${ }^{21}$, T. Koffas ${ }^{29}$, E. Koffeman ${ }^{105}$, L.A. Kogan ${ }^{118}$, S. Kohlmann ${ }^{175}$, F. Kohn ${ }^{54}$, Z. Kohout ${ }^{126}$, T. Kohriki ${ }^{65}$, T. Koi ${ }^{143}$, G.M. Kolachev ${ }^{107, *}$, H. Kolanoski ${ }^{16}$, V. Kolesnikov ${ }^{64}$, I. Koletsou ${ }^{89 a}$, J. Koll ${ }^{88}$, A.A. Komar ${ }^{94}$, Y. Komori ${ }^{155}$, T. Kondo ${ }^{65}$, T. Kono ${ }^{42, s}$, A.I. Kononov ${ }^{48}$, R. Konoplich ${ }^{108, t}$, N. Konstantinidis ${ }^{77}$, R. Kopeliansky ${ }^{152}$, S. Koperny ${ }^{38}$, K. Korcyl ${ }^{39}$, K. Kordas ${ }^{154}$, A. Korn ${ }^{118}$, A. Korol ${ }^{107}$, I. Korolkov ${ }^{12}$, E.V. Korolkova ${ }^{139}$, V.A. Korotkov ${ }^{128}$, O. Kortner ${ }^{99}$, S. Kortner ${ }^{99}$, V.V. Kostyukhin ${ }^{21}$, S. Kotov ${ }^{99}$, V.M. Kotov ${ }^{64}$, A. Kotwal ${ }^{45}$, C. Kourkoumelis ${ }^{9}$, V. Kouskoura ${ }^{154}$, A. Koutsman ${ }^{159 a}$, R. Kowalewski ${ }^{169}$, T.Z. Kowalski ${ }^{38}$, W. Kozanecki ${ }^{136}$, A.S. Kozhin ${ }^{128}$, V. Kral ${ }^{126}$, V.A. Kramarenko ${ }^{97}$, G. Kramberger ${ }^{74}$, M.W. Krasny ${ }^{78}$, A. Krasznahorkay ${ }^{108}$, J.K. Kraus ${ }^{21}$, S. Kreiss ${ }^{108}$, F. Krejci ${ }^{126}$, J. Kretzschmar ${ }^{73}$, N. Krieger ${ }^{54}$, P. Krieger ${ }^{158}$, K. Kroeninger ${ }^{54}$, H. Kroha ${ }^{99}$, J. Kroll ${ }^{120}$, J. Kroseberg ${ }^{21}$, J. Krstic ${ }^{13 a}$, U. Kruchonak ${ }^{64}$, H. Krüger ${ }^{21}$, T. Kruker ${ }^{17}$, N. Krumnack ${ }^{63}$, Z.V. Krumshteyn ${ }^{64}$, M.K. Kruse ${ }^{45}$, T. Kubota ${ }^{86}$, S. Kuday ${ }^{4 a}$, S. Kuehn ${ }^{48}$, A. Kugel ${ }^{58 c}$, T. Kuhl ${ }^{42}$, D. Kuhn ${ }^{61}$, V. Kukhtin ${ }^{64}$, Y. Kulchitsky ${ }^{90}$, S. Kuleshov ${ }^{32 b}$, C. Kummer ${ }^{98}$, M. Kuna ${ }^{78}$, J. Kunkle ${ }^{120}$, A. Kupco ${ }^{125}$, H. Kurashige ${ }^{66}$, M. Kurata ${ }^{160}$, Y.A. Kurochkin ${ }^{90}$, V. Kus ${ }^{125}$, E.S. Kuwertz ${ }^{147}$, M. Kuze ${ }^{157}$, J. Kvita ${ }^{142}$, R. Kwee ${ }^{16}$, A. La Rosa ${ }^{49}$, L. La Rotonda ${ }^{37 a, 37 b}$, L. Labarga ${ }^{80}$, J. Labbe ${ }^{5}$, S. Lablak ${ }^{135 a}$, C. Lacasta ${ }^{167}$, F. Lacava ${ }^{132 a, 132 b}$, J. Lacey ${ }^{29}$, H. Lacker ${ }^{16}$, D. Lacour ${ }^{78}$, V.R. Lacuesta ${ }^{167}$, E. Ladygin ${ }^{64}$, R. Lafaye $^{5}$, B. Laforge ${ }^{78}$, T. Lagouri ${ }^{176}$, S. Lai ${ }^{48}$, E. Laisne ${ }^{55}$, L. Lambourne ${ }^{77}$, C.L. Lampen ${ }^{7}$, W. Lampl ${ }^{7}$, E. Lancon ${ }^{136}$, U. Landgraf ${ }^{48}$, M.P.J. Landon ${ }^{75}$, V.S. Lang ${ }^{58 a}$, C. Lange ${ }^{42}$, A.J. Lankford ${ }^{163}$, F. Lanni ${ }^{25}$,

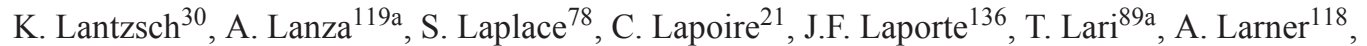
M. Lassnig ${ }^{30}$, P. Laurelli ${ }^{47}$, V. Lavorini ${ }^{37 a, 37 b}$, W. Lavrijsen ${ }^{15}$, P. Laycock ${ }^{73}$, O. Le Dortz ${ }^{78}$, E. Le Guirriec ${ }^{83}$, E. Le Menedeu ${ }^{12}$, T. LeCompte ${ }^{6}$, F. Ledroit-Guillon ${ }^{55}$, H. Lee ${ }^{105}$, J.S.H. Lee ${ }^{116}$, S.C. Lee ${ }^{151}$, L. Lee ${ }^{176}$, M. Lefebvre ${ }^{169}$, M. Legendre ${ }^{136}$, F. Legger ${ }^{98}$, C. Leggett ${ }^{15}$, M. Lehmacher ${ }^{21}$, G. Lehmann Miotto ${ }^{30}$, A.G. Leister ${ }^{176}$, M.A.L. Leite ${ }^{24 d}$, R. Leitner ${ }^{127}$, D. Lellouch ${ }^{172}$, B. Lemmer ${ }^{54}$, V. Lendermann ${ }^{58 a}$, K.J.C. Leney ${ }^{145 b}$, T. Lenz ${ }^{105}$, G. Lenzen ${ }^{175}$, B. Lenzi ${ }^{30}$, K. Leonhardt ${ }^{44}$, S. Leontsinis ${ }^{10}$, F. Lepold ${ }^{58 a}$, C. Leroy ${ }^{93}$, J-R. Lessard ${ }^{169}$, C.G. Lester ${ }^{28}$, C.M. Lester ${ }^{120}$, J. Levêque ${ }^{5}$, D. Levin ${ }^{87}$, L.J. Levinson ${ }^{172}$, A. Lewis ${ }^{118}$, G.H. Lewis ${ }^{108}$, A.M. Leyko ${ }^{21}$, M. Leyton ${ }^{16}$, B. Li ${ }^{83}$, H. Li $^{148}$,

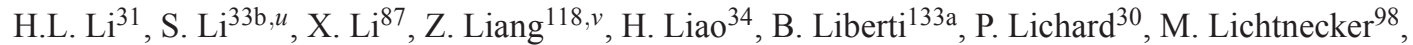

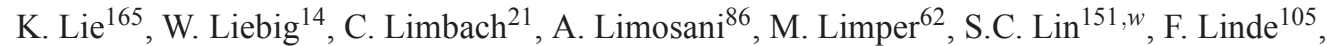
J.T. Linnemann ${ }^{88}$, E. Lipeles ${ }^{120}$, A. Lipniacka ${ }^{14}$, T.M. Liss ${ }^{165}$, D. Lissauer ${ }^{25}$, A. Lister ${ }^{49}$, A.M. Litke ${ }^{137}$,

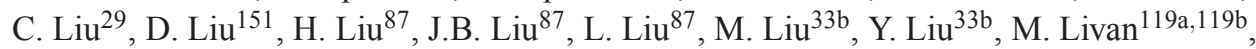


S.S.A. Livermore ${ }^{118}$, A. Lleres ${ }^{55}$, J. Llorente Merino ${ }^{80}$, S.L. Lloyd ${ }^{75}$, E. Lobodzinska ${ }^{42}$, P. Loch $^{7}$, W.S. Lockman ${ }^{137}$, T. Loddenkoetter ${ }^{21}$, F.K. Loebinger ${ }^{82}$, A. Loginov ${ }^{176}$, C.W. Loh ${ }^{168}$, T. Lohse ${ }^{16}$, K. Lohwasser ${ }^{48}$, M. Lokajicek ${ }^{125}$, V.P. Lombardo ${ }^{5}$, R.E. Long ${ }^{71}$, L. Lopes ${ }^{124 a}$, D. Lopez Mateos ${ }^{57}$, J. Lorenz ${ }^{98}$, N. Lorenzo Martinez ${ }^{115}$, M. Losada ${ }^{162}$, P. Loscutoff ${ }^{15}$, F. Lo Sterzo ${ }^{132 a, 132 b}$, M.J. Losty ${ }^{159 a, *}$, X. Lou ${ }^{41}$, A. Lounis ${ }^{115}$, K.F. Loureiro ${ }^{162}$, J. Love ${ }^{6}$, P.A. Love ${ }^{71}$, A.J. Lowe ${ }^{143, g}$, F. Lu $^{33 a}$, H.J. Lubatti ${ }^{138}$, C. Luci ${ }^{132 a, 132 b}$, A. Lucotte ${ }^{55}$, A. Ludwig ${ }^{44}$, D. Ludwig ${ }^{42}$, I. Ludwig ${ }^{48}$, J. Ludwig ${ }^{48}$, F. Luehring ${ }^{60}$, G. Luijckx ${ }^{105}$, W. Lukas ${ }^{61}$, L. Luminari1 ${ }^{132 a}$, E. Lund ${ }^{117}$, B. Lund-Jensen ${ }^{147}$, B. Lundberg ${ }^{79}$, J. Lundberg ${ }^{146 a, 146 b}$, O. Lundberg ${ }^{146 a, 146 b}$, J. Lundquist ${ }^{36}$, M. Lungwitz ${ }^{81}$, D. Lynn ${ }^{25}$, E. Lytken ${ }^{79}$, H. Ma ${ }^{25}$, L.L. Ma ${ }^{173}$, G. Maccarrone ${ }^{47}$, A. Macchiolo ${ }^{99}$, B. Maček ${ }^{74}$, J. Machado Miguens ${ }^{124 a}$, D. Macina ${ }^{30}$, R. Mackeprang ${ }^{36}$, R.J. Madaras ${ }^{15}$, H.J. Maddocks ${ }^{71}$, W.F. Mader $^{44}$, R. Maenner ${ }^{58 c}$, T. Maeno ${ }^{25}$, P. Mättig ${ }^{175}$, S. Mättig ${ }^{42}$, L. Magnoni1 ${ }^{163}$, E. Magradze ${ }^{54}$, K. Mahboubi ${ }^{48}$, J. Mahlstedt ${ }^{105}$, S. Mahmoud ${ }^{73}$, G. Mahout ${ }^{18}$, C. Maiani ${ }^{136}$, C. Maidantchik ${ }^{24 a}$, A. Maio ${ }^{124 a, c}$, S. Majewski ${ }^{25}$, Y. Makida ${ }^{65}$, N. Makovec ${ }^{115}$, P. Mal ${ }^{136}$, B. Malaescu ${ }^{30}$, Pa. Malecki ${ }^{39}$, P. Malecki ${ }^{39}$, V.P. Maleev ${ }^{121}$, F. Malek ${ }^{55}$, U. Mallik ${ }^{62}$, D. Malon ${ }^{6}$, C. Malone ${ }^{143}$, S. Maltezos ${ }^{10}$, V. Malyshev ${ }^{107}$, S. Malyukov ${ }^{30}$, R. Mameghani ${ }^{98}$, J. Mamuzic ${ }^{13 b}$, A. Manabe ${ }^{65}$, L. Mandelli ${ }^{89 a}$, I. Mandić ${ }^{74}$, R. Mandrysch ${ }^{16}$, J. Maneira ${ }^{124 a}$, A. Manfredini ${ }^{99}$, L. Manhaes de Andrade Filho ${ }^{24 b}$, J.A. Manjarres Ramos ${ }^{136}$, A. Mann ${ }^{54}$, P.M. Manning ${ }^{137}$, A. Manousakis-Katsikakis ${ }^{9}$, B. Mansoulie ${ }^{136}$, A. Mapelli ${ }^{30}$, L. Mapelli ${ }^{30}$, L. March ${ }^{167}$, J.F. Marchand ${ }^{29}$, F. Marchese ${ }^{133 a, 133 b}$, G. Marchiori ${ }^{78}$, M. Marcisovsky ${ }^{125}$, C.P. Marino ${ }^{169}$, F. Marroquim ${ }^{24 a}$, Z. Marshall ${ }^{30}$, F.K. Martens ${ }^{158}$, L.F. Marti ${ }^{17}$, S. Marti-Garcia ${ }^{167}$, B. $\operatorname{Martin}^{30}$, B. Martin ${ }^{88}$, J.P. Martin ${ }^{93}$, T.A. Martin ${ }^{18}$, V.J. Martin ${ }^{46}$, B. Martin dit Latour ${ }^{49}$, S. Martin-Haugh ${ }^{149}$, M. Martinez $^{12}$, V. Martinez Outschoorn ${ }^{57}$, A.C. Martyniuk ${ }^{169}$, M. Marx ${ }^{82}$, F. Marzano ${ }^{132 a}$, A. Marzin ${ }^{111}$, L. Masetti ${ }^{81}$, T. Mashimo ${ }^{155}$, R. Mashinistov ${ }^{94}$, J. Masik ${ }^{82}$, A.L. Maslennikov ${ }^{107}$, I. Massa ${ }^{20 a, 20 b}$, G. Massaro ${ }^{105}$, N. Massol ${ }^{5}$, P. Mastrandrea ${ }^{148}$, A. Mastroberardino ${ }^{37 a, 37 b}$, T. Masubuchi ${ }^{155}$, P. Matricon ${ }^{115}$, H. Matsunaga ${ }^{155}$, T. Matsushita ${ }^{66}$, C. Mattravers ${ }^{118, d}$, J. Maurer ${ }^{83}$, S.J. Maxfield ${ }^{73}$, D.A. Maximov ${ }^{107, h}$, A. Mayne ${ }^{139}$, R. Mazini ${ }^{151}$, M. Mazur $^{21}$, L. Mazzaferro ${ }^{133 a, 133 b}$, M. Mazzanti ${ }^{89 a}$, J. Mc Donald ${ }^{85}$, S.P. Mc Kee ${ }^{87}$, A. McCarn ${ }^{165}$, R.L. McCarthy ${ }^{148}$, T.G. McCarthy ${ }^{29}$, N.A. McCubbin ${ }^{129}$, K.W. McFarlane ${ }^{56, *}$, J.A. Mcfayden ${ }^{139}$, G. Mchedlidze ${ }^{51 b}$, T. Mclaughlan ${ }^{18}$, S.J. McMahon ${ }^{129}$, R.A. McPherson ${ }^{169, l}$, A. Meade ${ }^{84}$, J. Mechnich ${ }^{105}$, M. Mechtel ${ }^{175}$, M. Medinnis ${ }^{42}$, S. Meehan ${ }^{31}$, R. Meera-Lebbai ${ }^{111}$, T. Meguro ${ }^{116}$, S. Mehlhase ${ }^{36}$, A. Mehta ${ }^{73}$, K. Meier ${ }^{58 a}$, B. Meirose ${ }^{79}$, C. Melachrinos ${ }^{31}$, B.R. Mellado Garcia ${ }^{173}$, F. Meloni ${ }^{89 a, 89 b}$, L. Mendoza Navas ${ }^{162}$, Z. Meng ${ }^{151, x}$, A. Mengarelli ${ }^{20 a, 20 b}$, S. Menke ${ }^{99}$, E. Meoni ${ }^{161}$, K.M. Mercurio ${ }^{57}$, P. Mermod ${ }^{49}$, L. Merola ${ }^{102 a, 102 b}$, C. Meroni ${ }^{89 a}$, F.S. Merritt ${ }^{31}$, H. Merritt ${ }^{109}$, A. Messina ${ }^{30, y}$, J. Metcalfe ${ }^{25}$, A.S. Mete ${ }^{163}$, C. Meyer ${ }^{81}$, C. Meyer ${ }^{31}$, J-P. Meyer ${ }^{136}$, J. Meyer ${ }^{174}$, J. Meyer ${ }^{54}$, S. Michal ${ }^{30}$, L. Micu ${ }^{26 a}$, R.P. Middleton ${ }^{129}$, S. Migas ${ }^{73}$, L. Mijović ${ }^{136}$, G. Mikenberg ${ }^{172}$, M. Mikestikova ${ }^{125}$, M. Mikuž ${ }^{74}$, D.W. Miller ${ }^{31}$, R.J. Miller ${ }^{88}$, W.J. Mills ${ }^{168}$, C. Mills ${ }^{57}$, A. Milov ${ }^{172}$, D.A. Milstead ${ }^{146 a, 146 b}$, D. Milstein ${ }^{172}$, A.A. Minaenko ${ }^{128}$, M. Miñano Moya ${ }^{167}$, I.A. Minashvilii ${ }^{64}$, A.I. Mincer ${ }^{108}$, B. Mindur ${ }^{38}$, M. Mineev ${ }^{64}$, Y. Ming ${ }^{173}$, L.M. Mir ${ }^{12}$, G. Mirabelli ${ }^{132 a}$, J. Mitrevski ${ }^{137}$, V.A. Mitsou ${ }^{167}$, S. Mitsui ${ }^{65}$, P.S. Miyagawa ${ }^{139}$, J.U. Mjörnmark ${ }^{79}$, T. Moa ${ }^{146 a, 146 b}$, V. Moeller ${ }^{28}$, K. Mönig ${ }^{42}$, N. Möser ${ }^{21}$, S. Mohapatra ${ }^{148}$, W. Mohr ${ }^{48}$, R. Moles-Valls ${ }^{167}$, A. Molfetas ${ }^{30}$, J. Monk ${ }^{77}$, E. Monnier ${ }^{83}$, J. Montejo Berlingen ${ }^{12}$, F. Monticelli ${ }^{70}$, S. Monzani2 ${ }^{20 a}, 20 b$, R.W. Moore ${ }^{3}$, G.F. Moorhead ${ }^{86}$, C. Mora Herrera ${ }^{49}$, A. Moraes ${ }^{53}$, N. Morange ${ }^{136}$, J. Morel ${ }^{54}$, G. Morello ${ }^{37 a, 37 b}$, D. Moreno ${ }^{81}$, M. Moreno Llácer ${ }^{167}$, P. Morettini ${ }^{50 a}$, M. Morgenstern ${ }^{44}$, M. Morii ${ }^{57}$, A.K. Morley ${ }^{30}$, G. Mornacchi ${ }^{30}$, J.D. Morris ${ }^{75}$, L. Morvaj ${ }^{101}$, H.G. Moser ${ }^{99}$, M. Mosidze $^{51 b}$, J. Moss ${ }^{109}$, R. Mount ${ }^{143}$, E. Mountricha ${ }^{10, z}$, S.V. Mouraviev ${ }^{94, *}$, E.J.W. Moyse ${ }^{84}$, F. Mueller ${ }^{58 a}$, J. Mueller ${ }^{123}$, K. Mueller ${ }^{21}$, T.A. Müller ${ }^{98}$, T. Mueller ${ }^{81}$, D. Muenstermann ${ }^{30}$, Y. Munwes ${ }^{153}$, W.J. Murray ${ }^{129}$, I. Mussche ${ }^{105}$, E. Musto ${ }^{152}$, A.G. Myagkov ${ }^{128}$, M. Myska ${ }^{125}$, O. Nackenhorst ${ }^{54}$, J. Nadal ${ }^{12}$, K. Nagai ${ }^{160}$, R. Nagai ${ }^{157}$, K. Nagano ${ }^{65}$, A. Nagarkar ${ }^{109}$, Y. Nagasaka ${ }^{59}$, M. Nagel ${ }^{99}$, A.M. Nairz ${ }^{30}$, Y. Nakahama ${ }^{30}$, K. Nakamura ${ }^{155}$, T. Nakamura ${ }^{155}$, I. Nakano ${ }^{110}$, G. Nanava ${ }^{21}$, A. Napier ${ }^{161}$, R. Narayan ${ }^{58 b}$, M. Nash ${ }^{77, d}$, T. Nattermann ${ }^{21}$, T. Naumann ${ }^{42}$, G. Navarro ${ }^{162}$, H.A. Neal ${ }^{87}$, P.Yu. Nechaeva ${ }^{94}$, T.J. Neep ${ }^{82}$, A. Negri ${ }^{19 a, 119 b}$, G. Negri ${ }^{30}$, 
M. Negrini ${ }^{20 a}$, S. Nektarijevic ${ }^{49}$, A. Nelson ${ }^{163}$, T.K. Nelson ${ }^{143}$, S. Nemecek ${ }^{125}$, P. Nemethy ${ }^{108}$, A.A. Nepomuceno ${ }^{24 a}$, M. Nessi ${ }^{30, a a}$, M.S. Neubauer ${ }^{165}$, M. Neumann ${ }^{175}$, A. Neusiedl ${ }^{81}$, R.M. Neves ${ }^{108}$, P. Nevski ${ }^{25}$, F.M. Newcomer ${ }^{120}$, P.R. Newman ${ }^{18}$, V. Nguyen Thi Hong ${ }^{136}$, R.B. Nickerson ${ }^{118}$, R. Nicolaidou ${ }^{136}$, B. Nicquevert ${ }^{30}$, F. Niedercorn ${ }^{115}$, J. Nielsen ${ }^{137}$, N. Nikiforou ${ }^{35}$, A. Nikiforov ${ }^{16}$, V. Nikolaenko ${ }^{128}$, I. Nikolic-Audit ${ }^{78}$, K. Nikolics ${ }^{49}$, K. Nikolopoulos ${ }^{18}$, H. Nilsen $^{48}$, P. Nilsson ${ }^{8}$, Y. Ninomiya ${ }^{155}$, A. Nisati ${ }^{132 a}$, R. Nisius ${ }^{99}$, T. Nobe ${ }^{157}$, L. Nodulman ${ }^{6}$, M. Nomachi $^{116}$, I. Nomidis $^{154}$, S. Norberg ${ }^{11}$, M. Nordberg ${ }^{30}$, P.R. Norton ${ }^{129}$, J. Novakova ${ }^{127}$, M. Nozaki ${ }^{65}$, L. Nozka ${ }^{113}$, I.M. Nugent ${ }^{159 a}$, A.-E. Nuncio-Quiroz ${ }^{21}$, G. Nunes Hanninger ${ }^{86}$, T. Nunnemann ${ }^{98}$, E. Nurse ${ }^{77}$, B.J. O’Brien ${ }^{46}$, D.C. O’Neil ${ }^{142}$, V. O’Shea ${ }^{53}$, L.B. Oakes ${ }^{98}$, F.G. Oakham ${ }^{29, f}$, H. Oberlack ${ }^{99}$, J. Ocariz ${ }^{78}$, A. Ochi ${ }^{66}$, S. Oda ${ }^{69}$, S. Odaka ${ }^{65}$, J. Odier ${ }^{83}$, H. Ogren ${ }^{60}$, A. Oh ${ }^{82}$, S.H. Oh ${ }^{45}$, C.C. Ohm ${ }^{30}$, T. Ohshima ${ }^{101}$, W. Okamura ${ }^{116}$, H. Okawa ${ }^{25}$, Y. Okumura ${ }^{31}$, T. Okuyama ${ }^{155}$, A. Olariu ${ }^{26 a}$, A.G. Olchevski ${ }^{64}$, S.A. Olivares Pino ${ }^{32 a}$, M. Oliveira ${ }^{124 a, i}$, D. Oliveira Damazio ${ }^{25}$, E. Oliver Garcia ${ }^{167}$, D. Olivito ${ }^{120}$, A. Olszewski ${ }^{39}$, J. Olszowska ${ }^{39}$, A. Onofre ${ }^{124 a}, a b$, P.U.E. Onyisi ${ }^{31}$, C.J. Oram ${ }^{159 a}$, M.J. Oreglia ${ }^{31}$, Y. Oren ${ }^{153}$, D. Orestano ${ }^{134 a, 134 b}$, N. Orlando ${ }^{72 a, 72 b}$, I. Orlov ${ }^{107}$, C. Oropeza Barrera ${ }^{53}$, R.S. Orr ${ }^{158}$, B. Osculati ${ }^{50 a, 50 b}$, R. Ospanov ${ }^{120}$, C. Osuna ${ }^{12}$, G. Otero y Garzon ${ }^{27}$, J.P. Ottersbach ${ }^{105}$, M. Ouchrif ${ }^{135 d}$, E.A. Ouellette ${ }^{169}$, F. Ould-Saada ${ }^{117}$, A. Ouraou ${ }^{136}$, Q. Ouyang ${ }^{33 a}$, A. Ovcharova ${ }^{15}$, M. Owen ${ }^{82}$, S. Owen ${ }^{139}$, V.E. Ozcan ${ }^{19 a}$, N. Ozturk ${ }^{8}$, A. Pacheco Pages ${ }^{12}$, C. Padilla Aranda ${ }^{12}$, S. Pagan Griso ${ }^{15}$, E. Paganis ${ }^{139}$, C. Pahl ${ }^{99}$, F. Paige ${ }^{25}$, P. Pais ${ }^{84}$, K. Pajchel ${ }^{117}$, G. Palacino ${ }^{159 b}$, C.P. Paleari ${ }^{7}$, S. Palestini ${ }^{30}$, D. Pallin ${ }^{34}$, A. Palma ${ }^{124 a}$, J.D. Palmer ${ }^{18}$, Y.B. Pan $^{173}$, E. Panagiotopoulou ${ }^{10}$, J.G. Panduro Vazquez ${ }^{76}$, P. Pani ${ }^{105}$, N. Panikashvili ${ }^{87}$, S. Panitkin ${ }^{25}$, D. Pantea ${ }^{26 a}$, A. Papadelis ${ }^{146 a}$, Th.D. Papadopoulou ${ }^{10}$, A. Paramonov ${ }^{6}$, D. Paredes Hernandez ${ }^{34}$, W. Park ${ }^{25, a c}$, M.A. Parker ${ }^{28}$, F. Parodi ${ }^{50 a, 50 b}$, J.A. Parsons ${ }^{35}$, U. Parzefall ${ }^{48}$, S. Pashapour ${ }^{54}$, E. Pasqualucci132a , S. Passaggio ${ }^{50 a}$, A. Passeri ${ }^{134 a}$, F. Pastore ${ }^{134 a, 134 b, *}$, Fr. Pastore ${ }^{76}$, G. Pásztor ${ }^{49, a d}$, S. Pataraia ${ }^{175}$, N. Patel ${ }^{150}$, J.R. Pater ${ }^{82}$, S. Patricelli ${ }^{102 a, 102 b}$, T. Pauly ${ }^{30}$, M. Pecsy ${ }^{144 a}$, S. Pedraza Lopez ${ }^{167}$, M.I. Pedraza Morales ${ }^{173}$, S.V. Peleganchuk ${ }^{107}$, D. Pelikan ${ }^{166}$, H. Peng ${ }^{33 b}$, B. Penning ${ }^{31}$, A. Penson ${ }^{35}$, J. Penwell ${ }^{60}$, M. Perantoni2 ${ }^{24 a}$, K. Perez ${ }^{35, a e}$, T. Perez Cavalcanti ${ }^{42}$, E. Perez Codina ${ }^{159 a}$, M.T. Pérez García-Estañ ${ }^{167}$, V. Perez Reale ${ }^{35}$, L. Perini ${ }^{89 a, 89 b}$, H. Pernegger ${ }^{30}$, R. Perrino ${ }^{72 a}$, P. Perrodo ${ }^{5}$, V.D. Peshekhonov ${ }^{64}$, K. Peters ${ }^{30}$, B.A. Petersen ${ }^{30}$, J. Petersen ${ }^{30}$, T.C. Petersen ${ }^{36}$, E. Petit ${ }^{5}$, A. Petridis ${ }^{154}$, C. Petridou ${ }^{154}$, E. Petrolo ${ }^{132 a}$, F. Petrucci ${ }^{134 a}, 134 b$, D. Petschull ${ }^{42}$, M. Petteni ${ }^{142}$, R. Pezoa ${ }^{32 b}$, A. Phan ${ }^{86}$, P.W. Phillips ${ }^{129}$, G. Piacquadio ${ }^{30}$, A. Picazio ${ }^{49}$, E. Piccaro ${ }^{75}$, M. Piccinini ${ }^{20 a, 20 b}$, S.M. Piec ${ }^{42}$, R. Piegaia ${ }^{27}$, D.T. Pignotti ${ }^{109}$, J.E. Pilcher ${ }^{31}$, A.D. Pilkington ${ }^{82}$, J. Pina ${ }^{124 a, c}$, M. Pinamonti ${ }^{164 a, 164 c}$, A. Pinder ${ }^{118}$, J.L. Pinfold ${ }^{3}$, B. Pinto ${ }^{124 a}$, C. Pizio ${ }^{89 a, 89 b}$, M. Plamondon ${ }^{169}$, M.-A. Pleier ${ }^{25}$, E. Plotnikova ${ }^{64}$, A. Poblaguev ${ }^{25}$, S. Poddar ${ }^{58 a}$, F. Podlyski $^{34}$, L. Poggioli ${ }^{115}$, D. Pohl ${ }^{21}$, M. Pohl ${ }^{49}$, G. Polesello ${ }^{119 a}$, A. Policicchio ${ }^{37 a, 37 b}$, A. Polini ${ }^{20 a}$, J. Poll $^{75}$, V. Polychronakos ${ }^{25}$, D. Pomeroy ${ }^{23}$, K. Pommès ${ }^{30}$, L. Pontecorvo ${ }^{132 a}$, B.G. Pope ${ }^{88}$, G.A. Popeneciu ${ }^{26 a}$, D.S. Popovic ${ }^{13 a}$, A. Poppleton ${ }^{30}$, X. Portell Bueso ${ }^{30}$, G.E. Pospelov ${ }^{99}$, S. Pospisil ${ }^{126}$, I.N. Potrap ${ }^{99}$, C.J. Potter ${ }^{149}$, C.T. Potter ${ }^{14}$, G. Poulard ${ }^{30}$, J. Poveda ${ }^{60}$, V. Pozdnyakov ${ }^{64}$, R. Prabhu ${ }^{77}$, P. Pralavorio ${ }^{83}$,

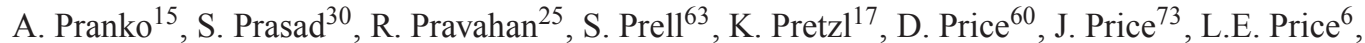

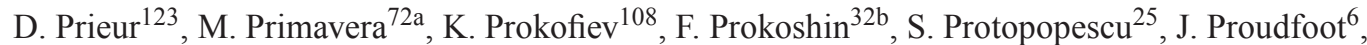
X. Prudent ${ }^{44}$, M. Przybycien ${ }^{38}$, H. Przysiezniak ${ }^{5}$, S. Psoroulas ${ }^{21}$, E. Ptacek ${ }^{114}$, E. Pueschel ${ }^{84}$, J. Purdham ${ }^{87}$, M. Purohit ${ }^{25, a c}$, P. Puzo ${ }^{115}$, Y. Pylypchenko ${ }^{62}$, J. Qian ${ }^{87}$, A. Quadt ${ }^{54}$, D.R. Quarrie ${ }^{15}$, W.B. Quayle ${ }^{173}$, F. Quinonez ${ }^{32 a}$, M. Raas ${ }^{104}$, V. Radeka ${ }^{25}$, V. Radescu ${ }^{42}$, P. Radloff $^{14}$, T. Rador ${ }^{19 a}$, F. Ragusa ${ }^{89 a, 89 b}$, G. Rahal ${ }^{178}$, A.M. Rahimi ${ }^{109}$, D. Rahm ${ }^{25}$, S. Rajagopalan ${ }^{25}$, M. Rammensee ${ }^{48}$, M. Rammes ${ }^{141}$, A.S. Randle-Conde ${ }^{40}$, K. Randrianarivony ${ }^{29}$, F. Rauscher ${ }^{98}$, T.C. Rave ${ }^{48}$, M. Raymond ${ }^{30}$, A.L. Read ${ }^{117}$, D.M. Rebuzzi ${ }^{119 a, 119 b}$, A. Redelbach ${ }^{174}$, G. Redlinger ${ }^{25}$, R. Reece ${ }^{120}$, K. Reeves ${ }^{41}$, A. Reinsch ${ }^{114}$, I. Reisinger ${ }^{43}$, C. Rembser ${ }^{30}$, Z.L. Ren ${ }^{151}$, A. Renaud ${ }^{115}$, M. Rescigno ${ }^{132 a}$, S. Resconi ${ }^{89 a}$, B. Resende ${ }^{136}$, P. Reznicek ${ }^{98}$, R. Rezvani ${ }^{158}$, R. Richter ${ }^{99}$, E. Richter-Was ${ }^{5}$,af, M. Ridel $^{78}$, M. Rijpstra ${ }^{105}$, M. Rijssenbeek ${ }^{148}$, A. Rimoldi119a,119b ${ }^{12 .}$ Rinaldi $^{20 a}$, R.R. Rios ${ }^{40}$, I. Riu ${ }^{12}$, G. Rivoltella ${ }^{89 a, 89 b}$, F. Rizatdinova ${ }^{112}$, E. Rizvi ${ }^{75}$, S.H. Robertson ${ }^{85, l}$, A. Robichaud-Veronneau ${ }^{118}$, D. Robinson ${ }^{28}$, 
J.E.M. Robinson ${ }^{82}$, A. Robson ${ }^{53}$, J.G. Rocha de Lima $^{106}$, C. Roda122a,122b, D. Roda Dos Santos ${ }^{30}$,

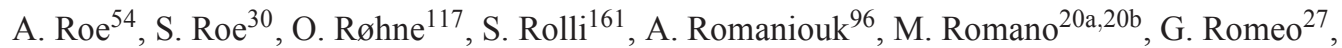
E. Romero Adam ${ }^{167}$, N. Rompotis ${ }^{138}$, L. Roos ${ }^{78}$, E. Ros ${ }^{167}$, S. Rosati ${ }^{132 a}$, K. Rosbach ${ }^{49}$, A. Rose $^{149}$, M. Rose $^{76}$, G.A. Rosenbaum ${ }^{158}$, E.I. Rosenberg ${ }^{63}$, P.L. Rosendahl ${ }^{14}$, O. Rosenthal ${ }^{141}$, L. Rosselet ${ }^{49}$, V. Rossetti ${ }^{12}$, E. Rossi ${ }^{132 a, 132 b}$, L.P. Rossi ${ }^{50 a}$, M. Rotaru ${ }^{26 a}$, I. Roth ${ }^{172}$, J. Rothberg ${ }^{138}$, D. Rousseau ${ }^{115}$, C.R. Royon ${ }^{136}$, A. Rozanov ${ }^{83}$, Y. Rozen ${ }^{152}$, X. Ruan ${ }^{33 a}, a g$, F. Rubbo ${ }^{12}$, I. Rubinskiy ${ }^{42}$, N. Ruckstuhl ${ }^{105}$, V.I. Rud ${ }^{97}$, C. Rudolph ${ }^{44}$, G. Rudolph ${ }^{61}$, F. Rühr ${ }^{7}$, A. Ruiz-Martinez ${ }^{63}$, L. Rumyantsev $^{64}$, Z. Rurikova ${ }^{48}$, N.A. Rusakovich ${ }^{64}$, A. Ruschke ${ }^{98}$, J.P. Rutherfoord ${ }^{7}$, P. Ruzicka ${ }^{125}$, Y.F. Ryabov ${ }^{121}$, M. Rybar ${ }^{127}$, G. Rybkin ${ }^{115}$, N.C. Ryder ${ }^{118}$, A.F. Saavedra ${ }^{150}$, I. Sadeh ${ }^{153}$, H.F-W. Sadrozinski ${ }^{137}$, R. Sadykov ${ }^{64}$, F. Safai Tehrani1 ${ }^{132 a}$, H. Sakamoto ${ }^{155}$, G. Salamanna ${ }^{75}$, A. Salamon ${ }^{133 a}$, M. Saleem ${ }^{111}$, D. Salek ${ }^{30}$, D. Salihagic ${ }^{99}$, A. Salnikov ${ }^{143}$, J. Salt ${ }^{167}$, B.M. Salvachua Ferrando ${ }^{6}$, D. Salvatore ${ }^{37 a, 37 b}$, F. Salvatore ${ }^{149}$, A. Salvucci ${ }^{104}$, A. Salzburger ${ }^{30}$, D. Sampsonidis ${ }^{154}$, B.H. Samset ${ }^{117}$, A. Sanchez ${ }^{102 a, 102 b}$, V. Sanchez Martinez ${ }^{167}$, H. Sandaker ${ }^{14}$, H.G. Sander ${ }^{81}$, M.P. Sanders ${ }^{98}$, M. Sandhoff ${ }^{175}$, T. Sandoval ${ }^{28}$, C. Sandoval ${ }^{162}$, R. Sandstroem ${ }^{99}$, D.P.C. Sankey ${ }^{129}$, A. Sansoni ${ }^{47}$, C. Santamarina Rios ${ }^{85}$, C. Santoni ${ }^{34}$, R. Santonico ${ }^{133 a, 133 b}$, H. Santos ${ }^{124 a}$, I. Santoyo Castillo ${ }^{149}$, J.G. Saraiva ${ }^{124 a}$, T. Sarangi ${ }^{173}$, E. Sarkisyan-Grinbaum ${ }^{8}$, B. Sarrazin ${ }^{21}$, F. Sarri122a,122b , G. Sartisohn ${ }^{175}$, O. Sasaki ${ }^{65}$, Y. Sasaki ${ }^{155}$, N. Sasao ${ }^{67}$, I. Satsounkevitch ${ }^{90}$, G. Sauvage ${ }^{5, *}$, E. Sauvan ${ }^{5}$, J.B. Sauvan ${ }^{115}$, P. Savard ${ }^{158, f}$, V. Savinov ${ }^{123}$, D.O. Savu ${ }^{30}$, L. Sawyer ${ }^{25, n}$, D.H. Saxon ${ }^{53}$, J. Saxon ${ }^{120}$, C. Sbarra ${ }^{20 a}$, A. Sbrizzi ${ }^{20 a, 20 b}$, D.A. Scannicchio ${ }^{163}$, M. Scarcella ${ }^{150}$, J. Schaarschmidt ${ }^{115}$, P. Schacht ${ }^{99}$, D. Schaefer ${ }^{120}$, U. Schäfer ${ }^{81}$, A. Schaelicke ${ }^{46}$, S. Schaepe ${ }^{21}$, S. Schaetzel ${ }^{58 b}$, A.C. Schaffer ${ }^{115}$, D. Schaile ${ }^{98}$, R.D. Schamberger ${ }^{148}$, A.G. Schamov ${ }^{107}$, V. Scharf ${ }^{58 a}$, V.A. Schegelsky ${ }^{121}$, D. Scheirich ${ }^{87}$, M. Schernau ${ }^{163}$, M.I. Scherzer ${ }^{35}$, C. Schiavi ${ }^{50 a, 50 b}$, J. Schieck ${ }^{98}$, M. Schioppa ${ }^{37 a, 37 b}$, S. Schlenker ${ }^{30}$, E. Schmidt ${ }^{48}$, K. Schmieden ${ }^{21}$, C. Schmitt ${ }^{81}$, S. Schmitt ${ }^{58 b}$, B. Schneider ${ }^{17}$, U. Schnoor ${ }^{44}$, L. Schoeffel ${ }^{136}$, A. Schoening ${ }^{58 b}$, A.L.S. Schorlemmer ${ }^{54}$, M. Schott ${ }^{30}$, D. Schouten ${ }^{159 a}$, J. Schovancova ${ }^{125}$, M. Schram ${ }^{85}$, C. Schroeder ${ }^{81}$, N. Schroer ${ }^{58 c}$, M.J. Schultens ${ }^{21}$, J. Schultes ${ }^{175}$, H.-C. Schultz-Coulon ${ }^{58 a}$, H. Schulz ${ }^{16}$, M. Schumacher ${ }^{48}$, B.A. Schumm ${ }^{137}$, Ph. Schune ${ }^{136}$, C. Schwanenberger ${ }^{82}$, A. Schwartzman ${ }^{143}$, Ph. Schwegler ${ }^{99}$, Ph. Schwemling ${ }^{78}$, R. Schwienhorst ${ }^{88}$, R. Schwierz ${ }^{44}$, J. Schwindling ${ }^{136}$, T. Schwindt ${ }^{21}$, M. Schwoerer ${ }^{5}$, F.G. Sciacca ${ }^{17}$, G. Sciolla ${ }^{23}$, W.G. Scott ${ }^{129}$, J. Searcy ${ }^{114}$, G. Sedov ${ }^{42}$, E. Sedykh ${ }^{121}$, S.C. Seidel ${ }^{103}$, A. Seiden ${ }^{137}$, F. Seifert ${ }^{44}$, J.M. Seixas ${ }^{24 a}$, G. Sekhniaidze ${ }^{102 a}$, S.J. Sekula ${ }^{40}$, K.E. Selbach ${ }^{46}$, D.M. Seliverstov ${ }^{121}$, B. Sellden ${ }^{146 a}$, G. Sellers ${ }^{73}$, M. Seman ${ }^{144 b}$, N. Semprini-Cesari ${ }^{20 a, 20 b}$, C. Serfon ${ }^{98}$, L. Serin ${ }^{115}$, L. Serkin ${ }^{54}$, R. Seuster ${ }^{159 a}$, H. Severini ${ }^{111}$, A. Sfyrla ${ }^{30}$, E. Shabalina ${ }^{54}$, M. Shamim ${ }^{114}$, L.Y. Shan ${ }^{33 a}$, J.T. Shank ${ }^{22}$, Q.T. Shao ${ }^{86}$, M. Shapiro ${ }^{15}$, P.B. Shatalov ${ }^{95}$, K. Shaw ${ }^{164 a, 164 c}$, D. Sherman ${ }^{176}$, P. Sherwood ${ }^{77}$, S. Shimizu ${ }^{101}$, M. Shimojima ${ }^{100}$, T. Shin ${ }^{56}$, M. Shiyakova ${ }^{64}$, A. Shmeleva ${ }^{94}$, M.J. Shochet ${ }^{31}$, D. Short ${ }^{118}$, S. Shrestha ${ }^{63}$, E. Shulga ${ }^{96}$, M.A. Shupe ${ }^{7}$, P. Sicho ${ }^{125}$, A. Sidoti ${ }^{132 a}$, F. Siegert ${ }^{48}$, Dj. Sijacki ${ }^{13 a}$, O. Silbert ${ }^{172}$, J. Silva ${ }^{124 a}$, Y. Silver ${ }^{153}$, D. Silverstein ${ }^{143}$, S.B. Silverstein ${ }^{146 a}$, V. Simak ${ }^{126}$, O. Simard ${ }^{136}$, Lj. Simic ${ }^{13 a}$, S. Simion ${ }^{115}$, E. Simioni ${ }^{81}$, B. Simmons ${ }^{77}$, R. Simoniello ${ }^{89 a, 89 b}$, M. Simonyan ${ }^{36}$, P. Sinervo ${ }^{158}$, N.B. Sinev ${ }^{114}$, V. Sipica ${ }^{141}$, G. Siragusa ${ }^{174}$, A. Sircar ${ }^{25}$, A.N. Sisakyan ${ }^{64, *}$, S.Yu. Sivoklokov ${ }^{97}$, J. Sjölin ${ }^{146 a, 146 b}$, T.B. Sjursen ${ }^{14}$, L.A. Skinnari ${ }^{15}$, H.P. Skottowe ${ }^{57}$, K. Skovpen ${ }^{107}$, P. Skubic ${ }^{111}$, M. Slater ${ }^{18}$, T. Slavicek ${ }^{126}$, K. Sliwa ${ }^{161}$, V. Smakhtin ${ }^{172}$, B.H. Smart ${ }^{46}$, L. Smestad ${ }^{117}$, S.Yu. Smirnov ${ }^{96}$, Y. Smirnov ${ }^{96}$, L.N. Smirnova ${ }^{97}$, O. Smirnova ${ }^{79}$, B.C. Smith $^{57}$, D. Smith ${ }^{143}$, K.M. Smith ${ }^{53}$, M. Smizanska ${ }^{71}$, K. Smolek ${ }^{126}$, A.A. Snesarev ${ }^{94}$, S.W. Snow ${ }^{82}$, J. Snow ${ }^{111}$, S. Snyder ${ }^{25}$, R. Sobie ${ }^{169, l}$, J. Sodomka ${ }^{126}$, A. Soffer ${ }^{153}$, C.A. Solans ${ }^{167}$, M. Solar ${ }^{126}$, J. Solc ${ }^{126}$, E. Yu. Soldatov ${ }^{96}$, U. Soldevila ${ }^{167}$, E. Solfaroli Camillocci132a,132b ${ }^{13 . A . ~ S o l o d k o v ~}{ }^{128}$, O.V. Solovyanov ${ }^{128}$, V. Solovyev ${ }^{121}$, N. Soni ${ }^{1}$, V. Sopko ${ }^{126}$, B. Sopko ${ }^{126}$, M. Sosebee ${ }^{8}$, R. Soualah ${ }^{164 a, 164 c}$, A. Soukharev ${ }^{107}$, S. Spagnolo ${ }^{72 a, 72 b}$, F. Spanò ${ }^{76}$, R. Spighi ${ }^{20 a}$, G. Spigo ${ }^{30}$, R. Spiwoks ${ }^{30}$, M. Spousta ${ }^{127, a h}$, T. Spreitzer ${ }^{158}$, B. Spurlock ${ }^{8}$, R.D. St. Denis ${ }^{53}$, J. Stahlman ${ }^{120}$, R. Stamen ${ }^{58 a}$, E. Stanecka ${ }^{39}$, R.W. Stanek ${ }^{6}$, C. Stanescu ${ }^{134 a}$, M. Stanescu-Bellu ${ }^{42}$, M.M. Stanitzki ${ }^{42}$, S. Stapnes ${ }^{117}$, E.A. Starchenko ${ }^{128}$, J. Stark ${ }^{55}$, P. Staroba ${ }^{125}$, P. Starovoitov ${ }^{42}$, R. Staszewski ${ }^{39}$, A. Staude ${ }^{98}$, P. Stavina ${ }^{144 a, *}$, G. Steele ${ }^{53}$, P. Steinbach ${ }^{44}$, P. Steinberg ${ }^{25}$, 
I. Stekl ${ }^{126}$, B. Stelzer ${ }^{142}$, H.J. Stelzer ${ }^{88}$, O. Stelzer-Chilton ${ }^{159 a}$, H. Stenzel ${ }^{52}$, S. Stern ${ }^{99}$, G.A. Stewart ${ }^{30}$, J.A. Stillings ${ }^{21}$, M.C. Stockton ${ }^{85}$, K. Stoerig ${ }^{48}$, G. Stoicea ${ }^{26 a}$, S. Stonjek ${ }^{99}$, P. Strachota ${ }^{127}$, A.R. Stradling ${ }^{8}$, A. Straessner ${ }^{44}$, J. Strandberg ${ }^{147}$, S. Strandberg ${ }^{146 a, 146 b}$, A. Strandlie ${ }^{117}$, M. Strang ${ }^{109}$, E. Strauss ${ }^{143}$, M. Strauss ${ }^{111}$, P. Strizenec ${ }^{144 b}$, R. Ströhmer ${ }^{174}$, D.M. Strom ${ }^{114}$, J.A. Strong ${ }^{76, *}$, R. Stroynowski ${ }^{40}$, B. Stugu ${ }^{14}$, I. Stumer ${ }^{25, *}$, J. Stupak ${ }^{148}$, P. Sturm ${ }^{175}$, N.A. Styles ${ }^{42}$, D.A. Soh ${ }^{151, v}$, D. Su ${ }^{143}$, HS. Subramania ${ }^{3}$, R. Subramaniam ${ }^{25}$, A. Succurro ${ }^{12}$, Y. Sugaya ${ }^{116}$, C. Suhr ${ }^{106}$, M. Suk ${ }^{127}$, V.V. Sulin ${ }^{94}$, S. Sultansoy ${ }^{4 d}$, T. Sumida ${ }^{67}$, X. Sun ${ }^{55}$, J.E. Sundermann ${ }^{48}$, K. Suruliz ${ }^{139}$, G. Susinno ${ }^{37 a, 37 b}$, M.R. Sutton ${ }^{149}$, Y. Suzuki ${ }^{65}$, Y. Suzuki ${ }^{66}$, M. Svatos ${ }^{125}$, S. Swedish ${ }^{168}$, I. Sykora ${ }^{144 a}$, T. Sykora ${ }^{127}$,

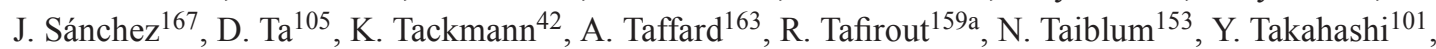
H. Takai ${ }^{25}$, R. Takashima ${ }^{68}$, H. Takeda ${ }^{66}$, T. Takeshita ${ }^{140}$, Y. Takubo ${ }^{65}$, M. Talby ${ }^{83}$, A. Talyshev ${ }^{107, h}$, M.C. Tamsett ${ }^{25}$, K.G. Tan ${ }^{86}$, J. Tanaka ${ }^{155}$, R. Tanaka ${ }^{115}$, S. Tanaka ${ }^{131}$, S. Tanaka ${ }^{65}$, A.J. Tanasijczuk ${ }^{142}$, K. Tani ${ }^{66}$, N. Tannoury ${ }^{83}$, S. Tapprogge ${ }^{81}$, D. Tardif ${ }^{158}$, S. Tarem ${ }^{152}$, F. Tarrade $^{29}$, G.F. Tartarelli ${ }^{89 a}$, P. Tas ${ }^{127}$, M. Tasevsky ${ }^{125}$, E. Tassi ${ }^{37 a, 37 b}$, Y. Tayalati ${ }^{135 d}$, C. Taylor ${ }^{77}$, F.E. Taylor ${ }^{92}$, G.N. Taylor ${ }^{86}$, W. Taylor ${ }^{159 b}$, M. Teinturier ${ }^{115}$, F.A. Teischinger ${ }^{30}$, M. Teixeira Dias Castanheira ${ }^{75}$, P. Teixeira-Dias ${ }^{76}$, K.K. Temming ${ }^{48}$, H. Ten Kate ${ }^{30}$, P.K. Teng ${ }^{151}$, S. Terada ${ }^{65}$, K. Terashi ${ }^{155}$, J. Terron ${ }^{80}$, M. Testa ${ }^{47}$, R.J. Teuscher ${ }^{158, l}$, J. Therhaag ${ }^{21}$, T. Theveneaux-Pelzer ${ }^{78}$, S. Thoma ${ }^{48}$, J.P. Thomas ${ }^{18}$, E.N. Thompson ${ }^{35}$, P.D. Thompson ${ }^{18}$, P.D. Thompson ${ }^{158}$, A.S. Thompson ${ }^{53}$, L.A. Thomsen ${ }^{36}$, E. Thomson ${ }^{120}$, M. Thomson ${ }^{28}$, W.M. Thong ${ }^{86}$, R.P. Thun ${ }^{87}$, F. Tian ${ }^{35}$, M.J. Tibbetts ${ }^{15}$, T. Tic ${ }^{125}$, V.O. Tikhomirov ${ }^{94}$, Y.A. Tikhonov ${ }^{107, h}$, S. Timoshenko ${ }^{96}$, E. Tiouchichine ${ }^{83}$, P. Tipton ${ }^{176}$, S. Tisserant ${ }^{83}$, T. Todorov $^{5}$, S. Todorova-Nova ${ }^{161}$,

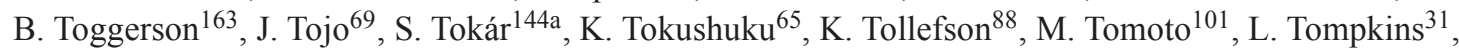
K. Toms ${ }^{103}$, A. Tonoyan ${ }^{14}$, C. Topfel ${ }^{17}$, N.D. Topilin ${ }^{64}$, E. Torrence ${ }^{114}$, H. Torres ${ }^{78}$, E. Torró Pastor ${ }^{167}$,

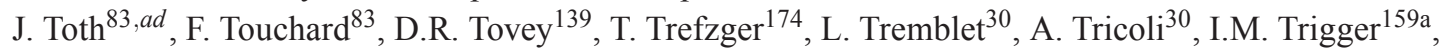
S. Trincaz-Duvoid ${ }^{78}$, M.F. Tripiana ${ }^{70}$, N. Triplett ${ }^{25}$, W. Trischuk ${ }^{158}$, B. Trocmé ${ }^{55}$, C. Troncon $^{89 a}$,

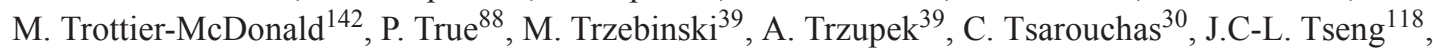

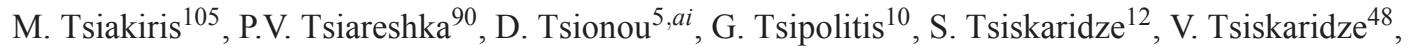
E.G. Tskhadadze ${ }^{51 \mathrm{a}}$, I.I. Tsukerman ${ }^{95}$, V. Tsulaia ${ }^{15}$, J.-W. Tsung ${ }^{21}$, S. Tsuno ${ }^{65}$, D. Tsybychev ${ }^{148}$, A. Tua ${ }^{139}$, A. Tudorache ${ }^{26 a}$, V. Tudorache ${ }^{26 a}$, J.M. Tuggle ${ }^{31}$, M. Turala ${ }^{39}$, D. Turecek ${ }^{126}$, I. Turk Cakir ${ }^{4 e}$, E. Turlay ${ }^{105}$, R. Turra ${ }^{89 a, 89 b}$, P.M. Tuts ${ }^{35}$, A. Tykhonov ${ }^{74}$, M. Tylmad ${ }^{146 a, 146 b}$, M. Tyndel ${ }^{129}$, G. Tzanakos $^{9}$, K. Uchida ${ }^{21}$, I. Ueda ${ }^{155}$, R. Ueno ${ }^{29}$, M. Ugland ${ }^{14}$, M. Uhlenbrock ${ }^{21}$, M. Uhrmacher ${ }^{54}$, F. Ukegawa ${ }^{160}$, G. Unal ${ }^{30}$, A. Undrus ${ }^{25}$, G. Unel ${ }^{163}$, Y. Unno ${ }^{65}$, D. Urbaniec ${ }^{35}$, P. Urquijo ${ }^{21}$, G. Usai ${ }^{8}$, M. Uslenghi ${ }^{119 a, 119 b}$, L. Vacavant ${ }^{83}$, V. Vacek ${ }^{126}$, B. Vachon ${ }^{85}$, S. Vahsen ${ }^{15}$, J. Valenta ${ }^{125}$, S. Valentinetti ${ }^{20 a, 20 b}$, A. Valero ${ }^{167}$, S. Valkar ${ }^{127}$, E. Valladolid Gallego ${ }^{167}$, S. Vallecorsa ${ }^{152}$, J.A. Valls Ferrer ${ }^{167}$, R. Van Berg ${ }^{120}$, P.C. Van Der Deij1 ${ }^{105}$, R. van der Geer ${ }^{105}$, H. van der Graaf ${ }^{105}$, R. Van Der Leeuw ${ }^{105}$, E. van der Poel ${ }^{105}$, D. van der $\operatorname{Ster}^{30}$, N. van Eldik ${ }^{30}$, P. van Gemmeren ${ }^{6}$, I. van Vulpen ${ }^{105}$, M. Vanadia ${ }^{99}$, W. Vandelli ${ }^{30}$,

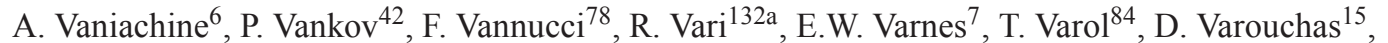
A. Vartapetian ${ }^{8}$, K.E. Varvell ${ }^{150}$, V.I. Vassilakopoulos ${ }^{56}$, F. Vazeille ${ }^{34}$, T. Vazquez Schroeder ${ }^{54}$,

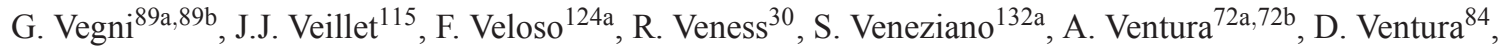
M. Venturi ${ }^{48}$, N. Venturi ${ }^{158}$, V. Vercesi ${ }^{119 a}$, M. Verducci ${ }^{138}$, W. Verkerke ${ }^{105}$, J.C. Vermeulen ${ }^{105}$, A. Vest ${ }^{44}$, M.C. Vetterli ${ }^{142, f}$, I. Vichou ${ }^{165}$, T. Vickey ${ }^{145 b, a j}$, O.E. Vickey Boeriu ${ }^{145 b}$, G.H.A. Viehhauser ${ }^{18}$, S. Viel $^{168}$, M. Villa 20a,20b, M. Villaplana Perez ${ }^{167}$, E. Vilucchi ${ }^{47}$, M.G. Vincter ${ }^{29}$, E. Vinek $^{30}$, V.B. Vinogradov ${ }^{64}$, M. Virchaux ${ }^{136, *}$, J. Virzi ${ }^{15}$, O. Vitells ${ }^{172}$, M. Viti ${ }^{42}$, I. Vivarelli ${ }^{48}$, F. Vives Vaque ${ }^{3}$, S. Vlachos ${ }^{10}$,

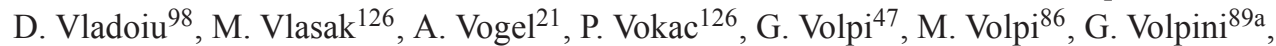
H. von der Schmitt ${ }^{99}$, H. von Radziewski ${ }^{48}$, E. von Toerne ${ }^{21}$, V. Vorobel ${ }^{127}$, V. Vorwerk ${ }^{12}$, M. Vos $^{167}$, R. Voss $^{30}$, J.H. Vossebeld ${ }^{73}$, N. Vranjes ${ }^{136}$, M. Vranjes Milosavljevic ${ }^{105}$, V. Vrba ${ }^{125}$, M. Vreeswijk ${ }^{105}$, T. Vu Anh ${ }^{48}$, R. Vuillermet ${ }^{30}$, I. Vukotic ${ }^{31}$, W. Wagner ${ }^{175}$, P. Wagner ${ }^{120}$, H. Wahlen ${ }^{175}$, S. Wahrmund ${ }^{44}$, J. Wakabayashi ${ }^{101}, \mathrm{~S}$. Walch ${ }^{87}$, J. Walder ${ }^{71}$, R. Walker ${ }^{98}$, W. Walkowiak ${ }^{141}$, R. Wall ${ }^{176}$, P. Waller ${ }^{73}$,

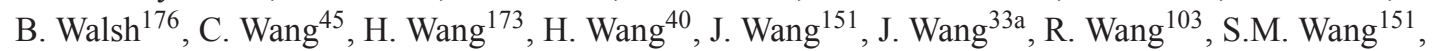
T. Wang ${ }^{21}$, A. Warburton ${ }^{85}$, C.P. Ward ${ }^{28}$, D.R. Wardrope ${ }^{77}$, M. Warsinsky ${ }^{48}$, A. Washbrook ${ }^{46}$, 
C. Wasicki ${ }^{42}$, I. Watanabe ${ }^{66}$, P.M. Watkins ${ }^{18}$, A.T. Watson ${ }^{18}$, I.J. Watson ${ }^{150}$, M.F. Watson ${ }^{18}$, G. Watts ${ }^{138}$, S. Watts ${ }^{82}$, A.T. Waugh ${ }^{150}$, B.M. Waugh ${ }^{77}$, M.S. Weber ${ }^{17}$, P. Weber ${ }^{54}$, J.S. Webster ${ }^{31}$, A.R. Weidberg ${ }^{118}$, P. Weigell ${ }^{99}$, J. Weingarten ${ }^{54}$, C. Weiser ${ }^{48}$, P.S. Wells ${ }^{30}$, T. Wenaus ${ }^{25}$, D. Wendland ${ }^{16}$, Z. Weng ${ }^{151, v}$, T. Wengler ${ }^{30}$, S. Wenig ${ }^{30}$, N. Wermes ${ }^{21}$, M. Werner ${ }^{48}$, P. Werner ${ }^{30}$, M. Werth ${ }^{163}$, M. Wessels ${ }^{58 a}$, J. Wetter ${ }^{161}$, C. Weydert ${ }^{55}$, K. Whalen ${ }^{29}$, A. White ${ }^{8}$, M.J. White ${ }^{86}$, S. White ${ }^{122 a, 122 b}$, S.R. Whitehead ${ }^{118}$, D. Whiteson ${ }^{163}$, D. Whittington ${ }^{60}$, F. Wicek ${ }^{115}$, D. Wicke ${ }^{175}$, F.J. Wickens ${ }^{129}$, W. Wiedenmann ${ }^{173}$, M. Wielers ${ }^{129}$, P. Wienemann ${ }^{21}$, C. Wiglesworth ${ }^{75}$, L.A.M. Wiik-Fuchs ${ }^{21}$, P.A. Wijeratne ${ }^{77}$, A. Wildauer ${ }^{99}$, M.A. Wildt ${ }^{42, s}$, I. Wilhelm ${ }^{127}$, H.G. Wilkens ${ }^{30}$, J.Z. Will ${ }^{98}$, E. Williams ${ }^{35}$, H.H. Williams ${ }^{120}$, W. Willis ${ }^{35}$, S. Willocq ${ }^{84}$, J.A. Wilson ${ }^{18}$, M.G. Wilson ${ }^{143}$, A. Wilson ${ }^{87}$, I. Wingerter-Seez ${ }^{5}$, S. Winkelmann ${ }^{48}$, F. Winklmeier ${ }^{30}$, M. Wittgen ${ }^{143}$, S.J. Wollstadt ${ }^{81}$, M.W. Wolter ${ }^{39}$, H. Wolters ${ }^{124 a, i}$, W.C. Wong ${ }^{41}$, G. Wooden ${ }^{87}$, B.K. Wosiek ${ }^{39}$, J. Wotschack ${ }^{30}$, M.J. Woudstra ${ }^{82}$, K.W. Wozniak ${ }^{39}$, K. Wraight ${ }^{53}$,

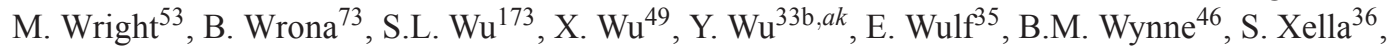
M. Xiao ${ }^{136}, \mathrm{~S} . \mathrm{Xie}^{48}$, C. Xu ${ }^{33 b, z}$, D. Xu ${ }^{139}$, L. Xu ${ }^{33 b}$, B. Yabsley ${ }^{150}$, S. Yacoob ${ }^{145 a, a l}$, M. Yamada ${ }^{65}$, H. Yamaguchi ${ }^{155}$, A. Yamamoto ${ }^{65}$, K. Yamamoto ${ }^{63}$, S. Yamamoto ${ }^{155}$, T. Yamamura ${ }^{155}$, T. Yamanaka ${ }^{155}$,

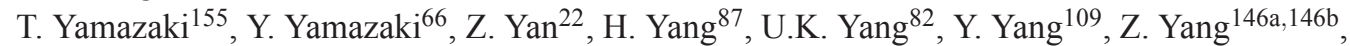

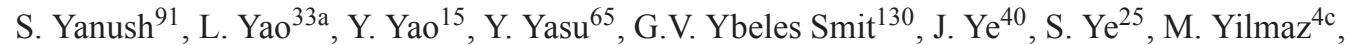

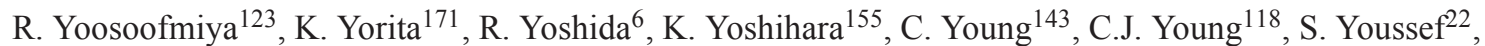

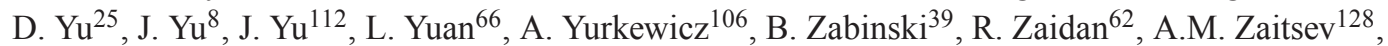
Z. Zajacova ${ }^{30}$, L. Zanello ${ }^{132 a, 132 b}$, D. Zanzi ${ }^{99}$, A. Zaytsev ${ }^{25}$, C. Zeitnitz ${ }^{175}$, M. Zeman ${ }^{126}$, A. Zemla ${ }^{39}$, C. Zendler ${ }^{21}$, O. Zenin ${ }^{128}$, T. Ženišs ${ }^{14 a}$, Z. Zinonos ${ }^{122 a, 122 b}$, S. Zenz ${ }^{15}$, D. Zerwas ${ }^{115}$, G. Zevi della Porta ${ }^{57}$, D. Zhang ${ }^{33 b, a m}$, H. Zhang ${ }^{88}$, J. Zhang ${ }^{6}$, X. Zhang ${ }^{33 d}$, Z. Zhang ${ }^{115}$, L. Zhao ${ }^{108}$, Z. Zhao ${ }^{33 b}$,

A. Zhemchugov ${ }^{64}$, J. Zhong ${ }^{18}$, B. Zhou ${ }^{87}$, N. Zhou ${ }^{163}$, Y. Zhou ${ }^{151}$, C.G. Zhu ${ }^{33 d}$, H. Zhu ${ }^{42}$, J. Zhu ${ }^{87}$, Y. Zhu ${ }^{33 b}$, X. Zhuang ${ }^{98}$, V. Zhuravlov ${ }^{99}$, A. Zibell ${ }^{98}$, D. Zieminska ${ }^{60}$, N.I. Zimin ${ }^{64}$, R. Zimmermann ${ }^{21}$, S. Zimmermann ${ }^{21}$, S. Zimmermann ${ }^{48}$, M. Ziolkowski ${ }^{141}$, R. Zitoun ${ }^{5}$, L. Živković ${ }^{35}$, V.V. Zmouchko ${ }^{128, *}$, G. Zobernig ${ }^{173}$, A. Zoccoli ${ }^{20 a, 20 b}$, M. zur Nedden ${ }^{16}$, V. Zutshi ${ }^{106}$, L. Zwalinski ${ }^{30}$

1 School of Chemistry and Physics, University of Adelaide, Adelaide, Australia

2 Physics Department, SUNY Albany, Albany NY, United States of America

3 Department of Physics, University of Alberta, Edmonton AB, Canada

$4{ }^{(a)}$ Department of Physics, Ankara University, Ankara; ${ }^{(b)}$ Department of Physics, Dumlupinar University, Kutahya; ${ }^{(c)}$ Department of Physics, Gazi University, Ankara; ${ }^{(d)}$ Division of Physics, TOBB University of Economics and Technology, Ankara; ${ }^{(e)}$ Turkish Atomic Energy Authority, Ankara, Turkey

5 LAPP, CNRS/IN2P3 and Université de Savoie, Annecy-le-Vieux, France

6 High Energy Physics Division, Argonne National Laboratory, Argonne IL, United States of America

7 Department of Physics, University of Arizona, Tucson AZ, United States of America

8 Department of Physics, The University of Texas at Arlington, Arlington TX, United States of America

9 Physics Department, University of Athens, Athens, Greece

10 Physics Department, National Technical University of Athens, Zografou, Greece

11 Institute of Physics, Azerbaijan Academy of Sciences, Baku, Azerbaijan

12 Institut de Física d'Altes Energies and Departament de Física de la Universitat Autònoma de Barcelona and ICREA, Barcelona, Spain

13 (a) Institute of Physics, University of Belgrade, Belgrade; (b) Vinca Institute of Nuclear Sciences, University of Belgrade, Belgrade, Serbia

14 Department for Physics and Technology, University of Bergen, Bergen, Norway

15 Physics Division, Lawrence Berkeley National Laboratory and University of California, Berkeley CA, United States of America

16 Department of Physics, Humboldt University, Berlin, Germany

17 Albert Einstein Center for Fundamental Physics and Laboratory for High Energy Physics, University of Bern, Bern, Switzerland

18 School of Physics and Astronomy, University of Birmingham, Birmingham, United Kingdom 
19 (a) Department of Physics, Bogazici University, Istanbul; (b) Division of Physics, Dogus University, Istanbul;

${ }^{(c)}$ Department of Physics Engineering, Gaziantep University, Gaziantep; ${ }^{(d)}$ Department of Physics, Istanbul Technical University, Istanbul, Turkey

$20{ }^{(a)}$ INFN Sezione di Bologna; ${ }^{(b)}$ Dipartimento di Fisica, Università di Bologna, Bologna, Italy

21 Physikalisches Institut, University of Bonn, Bonn, Germany

22 Department of Physics, Boston University, Boston MA, United States of America

23 Department of Physics, Brandeis University, Waltham MA, United States of America

24 (a) Universidade Federal do Rio De Janeiro COPPE/EE/IF, Rio de Janeiro; ${ }^{(b)}$ Federal University of Juiz de Fora (UFJF), Juiz de Fora; ${ }^{(c)}$ Federal University of Sao Joao del Rei (UFSJ), Sao Joao del Rei; ${ }^{(d)}$ Instituto de Fisica, Universidade de Sao Paulo, Sao Paulo, Brazil

25 Physics Department, Brookhaven National Laboratory, Upton NY, United States of America

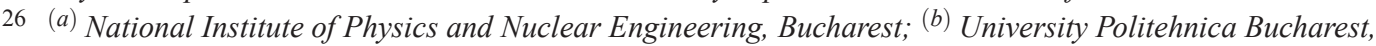
Bucharest; ${ }^{(c)}$ West University in Timisoara, Timisoara, Romania

27 Departamento de Física, Universidad de Buenos Aires, Buenos Aires, Argentina

28 Cavendish Laboratory, University of Cambridge, Cambridge, United Kingdom

29 Department of Physics, Carleton University, Ottawa ON, Canada

30 CERN, Geneva, Switzerland

31 Enrico Fermi Institute, University of Chicago, Chicago IL, United States of America

32 (a) Departamento de Física, Pontificia Universidad Católica de Chile, Santiago; ${ }^{(b)}$ Departamento de Física, Universidad Técnica Federico Santa María, Valparaíso, Chile

33 (a) Institute of High Energy Physics, Chinese Academy of Sciences, Beijing; (b) Department of Modern Physics, University of Science and Technology of China, Anhui; ${ }^{(c)}$ Department of Physics, Nanjing University, Jiangsu; (d) School of Physics, Shandong University, Shandong; (e) Physics Department, Shanghai Jiao Tong University, Shanghai, China

34 Laboratoire de Physique Corpusculaire, Clermont Université and Université Blaise Pascal and CNRS/IN2P3, Clermont-Ferrand, France

35 Nevis Laboratory, Columbia University, Irvington NY, United States of America

36 Niels Bohr Institute, University of Copenhagen, Kobenhavn, Denmark

37 (a) INFN Gruppo Collegato di Cosenza; ${ }^{(b)}$ Dipartimento di Fisica, Università della Calabria, Arcavata di Rende, Italy

38 AGH University of Science and Technology, Faculty of Physics and Applied Computer Science, Krakow, Poland

39 The Henryk Niewodniczanski Institute of Nuclear Physics, Polish Academy of Sciences, Krakow, Poland

40 Physics Department, Southern Methodist University, Dallas TX, United States of America

41 Physics Department, University of Texas at Dallas, Richardson TX, United States of America

42 DESY, Hamburg and Zeuthen, Germany

43 Institut für Experimentelle Physik IV, Technische Universität Dortmund, Dortmund, Germany

44 Institut für Kern- und Teilchenphysik, Technical University Dresden, Dresden, Germany

45 Department of Physics, Duke University, Durham NC, United States of America

46 SUPA - School of Physics and Astronomy, University of Edinburgh, Edinburgh, United Kingdom

47 INFN Laboratori Nazionali di Frascati, Frascati, Italy

48 Fakultät für Mathematik und Physik, Albert-Ludwigs-Universität, Freiburg, Germany

49 Section de Physique, Université de Genève, Geneva, Switzerland

50 (a) INFN Sezione di Genova; ${ }^{(b)}$ Dipartimento di Fisica, Università di Genova, Genova, Italy

51 (a) E. Andronikashvili Institute of Physics, Iv. Javakhishvili Tbilisi State University, Tbilisi; ${ }^{(b)}$ High Energy Physics Institute, Tbilisi State University, Tbilisi, Georgia

52 II Physikalisches Institut, Justus-Liebig-Universität Giessen, Giessen, Germany

53 SUPA - School of Physics and Astronomy, University of Glasgow, Glasgow, United Kingdom

54 II Physikalisches Institut, Georg-August-Universität, Göttingen, Germany

55 Laboratoire de Physique Subatomique et de Cosmologie, Université Joseph Fourier and CNRS/IN2P3 and Institut National Polytechnique de Grenoble, Grenoble, France

56 Department of Physics, Hampton University, Hampton VA, United States of America

57 Laboratory for Particle Physics and Cosmology, Harvard University, Cambridge MA, United States of America 
58 (a) Kirchhoff-Institut für Physik, Ruprecht-Karls-Universität Heidelberg, Heidelberg; (b) Physikalisches Institut, Ruprecht-Karls-Universität Heidelberg, Heidelberg; ${ }^{(c)}$ ZITI Institut für technische Informatik,

Ruprecht-Karls-Universität Heidelberg, Mannheim, Germany

59 Faculty of Applied Information Science, Hiroshima Institute of Technology, Hiroshima, Japan

60 Department of Physics, Indiana University, Bloomington IN, United States of America

61 Institut für Astro- und Teilchenphysik, Leopold-Franzens-Universität, Innsbruck, Austria

62 University of Iowa, Iowa City IA, United States of America

63 Department of Physics and Astronomy, Iowa State University, Ames IA, United States of America

64 Joint Institute for Nuclear Research, JINR Dubna, Dubna, Russia

65 KEK, High Energy Accelerator Research Organization, Tsukuba, Japan

66 Graduate School of Science, Kobe University, Kobe, Japan

67 Faculty of Science, Kyoto University, Kyoto, Japan

68 Kyoto University of Education, Kyoto, Japan

69 Department of Physics, Kyushu University, Fukuoka, Japan

70 Instituto de Física La Plata, Universidad Nacional de La Plata and CONICET, La Plata, Argentina

71 Physics Department, Lancaster University, Lancaster, United Kingdom

72 (a) INFN Sezione di Lecce; (b) Dipartimento di Matematica e Fisica, Università del Salento, Lecce, Italy

73 Oliver Lodge Laboratory, University of Liverpool, Liverpool, United Kingdom

74 Department of Physics, Jožef Stefan Institute and University of Ljubljana, Ljubljana, Slovenia

75 School of Physics and Astronomy, Queen Mary University of London, London, United Kingdom

76 Department of Physics, Royal Holloway University of London, Surrey, United Kingdom

77 Department of Physics and Astronomy, University College London, London, United Kingdom

78 Laboratoire de Physique Nucléaire et de Hautes Energies, UPMC and Université Paris-Diderot and CNRS/IN2P3, Paris, France

79 Fysiska institutionen, Lunds universitet, Lund, Sweden

80 Departamento de Fisica Teorica C-15, Universidad Autonoma de Madrid, Madrid, Spain

81 Institut für Physik, Universität Mainz, Mainz, Germany

82 School of Physics and Astronomy, University of Manchester, Manchester, United Kingdom

83 CPPM, Aix-Marseille Université and CNRS/IN2P3, Marseille, France

84 Department of Physics, University of Massachusetts, Amherst MA, United States of America

85 Department of Physics, McGill University, Montreal QC, Canada

86 School of Physics, University of Melbourne, Victoria, Australia

87 Department of Physics, The University of Michigan, Ann Arbor MI, United States of America

88 Department of Physics and Astronomy, Michigan State University, East Lansing MI, United States of America

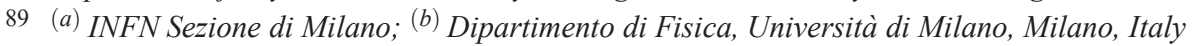

90 B.I. Stepanov Institute of Physics, National Academy of Sciences of Belarus, Minsk, Republic of Belarus

91 National Scientific and Educational Centre for Particle and High Energy Physics, Minsk, Republic of Belarus

92 Department of Physics, Massachusetts Institute of Technology, Cambridge MA, United States of America

93 Group of Particle Physics, University of Montreal, Montreal QC, Canada

94 P.N. Lebedev Institute of Physics, Academy of Sciences, Moscow, Russia

95 Institute for Theoretical and Experimental Physics (ITEP), Moscow, Russia

96 Moscow Engineering and Physics Institute (MEPhI), Moscow, Russia

97 Skobeltsyn Institute of Nuclear Physics, Lomonosov Moscow State University, Moscow, Russia

98 Fakultät für Physik, Ludwig-Maximilians-Universität München, München, Germany

99 Max-Planck-Institut für Physik (Werner-Heisenberg-Institut), München, Germany

100 Nagasaki Institute of Applied Science, Nagasaki, Japan

101 Graduate School of Science and Kobayashi-Maskawa Institute, Nagoya University, Nagoya, Japan

$102{ }^{(a)}$ INFN Sezione di Napoli; ${ }^{(b)}$ Dipartimento di Scienze Fisiche, Università di Napoli, Napoli, Italy

103 Department of Physics and Astronomy, University of New Mexico, Albuquerque NM, United States of America

104 Institute for Mathematics, Astrophysics and Particle Physics, Radboud University Nijmegen/Nikhef, Nijmegen, Netherlands

105 Nikhef National Institute for Subatomic Physics and University of Amsterdam, Amsterdam, Netherlands 
106 Department of Physics, Northern Illinois University, DeKalb IL, United States of America

107 Budker Institute of Nuclear Physics, SB RAS, Novosibirsk, Russia

108 Department of Physics, New York University, New York NY, United States of America

109 Ohio State University, Columbus OH, United States of America

110 Faculty of Science, Okayama University, Okayama, Japan

111 Homer L. Dodge Department of Physics and Astronomy, University of Oklahoma, Norman OK, United States of America

112 Department of Physics, Oklahoma State University, Stillwater OK, United States of America

113 Palacký University, RCPTM, Olomouc, Czech Republic

114 Center for High Energy Physics, University of Oregon, Eugene OR, United States of America

115 LAL, Université Paris-Sud and CNRS/IN2P3, Orsay, France

116 Graduate School of Science, Osaka University, Osaka, Japan

117 Department of Physics, University of Oslo, Oslo, Norway

118 Department of Physics, Oxford University, Oxford, United Kingdom

119 (a) INFN Sezione di Pavia; (b) Dipartimento di Fisica, Università di Pavia, Pavia, Italy

120 Department of Physics, University of Pennsylvania, Philadelphia PA, United States of America

121 Petersburg Nuclear Physics Institute, Gatchina, Russia

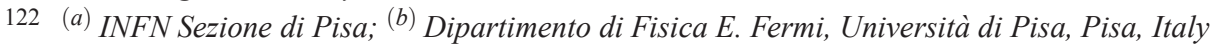

123 Department of Physics and Astronomy, University of Pittsburgh, Pittsburgh PA, United States of America

124 (a) Laboratorio de Instrumentacao e Fisica Experimental de Particulas - LIP, Lisboa, Portugal;

(b) Departamento de Fisica Teorica y del Cosmos and CAFPE, Universidad de Granada, Granada, Spain

125 Institute of Physics, Academy of Sciences of the Czech Republic, Praha, Czech Republic

126 Czech Technical University in Prague, Praha, Czech Republic

127 Faculty of Mathematics and Physics, Charles University in Prague, Praha, Czech Republic

128 State Research Center Institute for High Energy Physics, Protvino, Russia

129 Particle Physics Department, Rutherford Appleton Laboratory, Didcot, United Kingdom

130 Physics Department, University of Regina, Regina SK, Canada

131 Ritsumeikan University, Kusatsu, Shiga, Japan

132 (a) INFN Sezione di Roma I; ${ }^{(b)}$ Dipartimento di Fisica, Università La Sapienza, Roma, Italy

133 (a) INFN Sezione di Roma Tor Vergata; ${ }^{(b)}$ Dipartimento di Fisica, Università di Roma Tor Vergata, Roma, Italy

134 (a) INFN Sezione di Roma Tre; ${ }^{(b)}$ Dipartimento di Fisica, Università Roma Tre, Roma, Italy

135 (a) Faculté des Sciences Ain Chock, Réseau Universitaire de Physique des Hautes Energies - Université Hassan

II, Casablanca; ${ }^{(b)}$ Centre National de l'Energie des Sciences Techniques Nucleaires, Rabat; ${ }^{(c)}$ Faculté des Sciences Semlalia, Université Cadi Ayyad, LPHEA-Marrakech; ${ }^{(d)}$ Faculté des Sciences, Université Mohamed Premier and LPTPM, Oujda; ${ }^{(e)}$ Faculté des sciences, Université Mohammed V-Agdal, Rabat, Morocco

136 DSM/IRFU (Institut de Recherches sur les Lois Fondamentales de l'Univers), CEA Saclay (Commissariat à l'Energie Atomique et aux Energies Alternatives), Gif-sur-Yvette, France

137 Santa Cruz Institute for Particle Physics, University of California Santa Cruz, Santa Cruz CA, United States of America

138 Department of Physics, University of Washington, Seattle WA, United States of America

139 Department of Physics and Astronomy, University of Sheffield, Sheffield, United Kingdom

140 Department of Physics, Shinshu University, Nagano, Japan

141 Fachbereich Physik, Universität Siegen, Siegen, Germany

142 Department of Physics, Simon Fraser University, Burnaby BC, Canada

143 SLAC National Accelerator Laboratory, Stanford CA, United States of America

144 (a) Faculty of Mathematics, Physics \& Informatics, Comenius University, Bratislava; ${ }^{(b)}$ Department of Subnuclear Physics, Institute of Experimental Physics of the Slovak Academy of Sciences, Kosice, Slovak Republic

145 (a) Department of Physics, University of Johannesburg, Johannesburg; ${ }^{(b)}$ School of Physics, University of the Witwatersrand, Johannesburg, South Africa

146 (a) Department of Physics, Stockholm University; (b) The Oskar Klein Centre, Stockholm, Sweden

147 Physics Department, Royal Institute of Technology, Stockholm, Sweden 
Departments of Physics \& Astronomy and Chemistry, Stony Brook University, Stony Brook NY, United States of America

149 Department of Physics and Astronomy, University of Sussex, Brighton, United Kingdom

150 School of Physics, University of Sydney, Sydney, Australia

151 Institute of Physics, Academia Sinica, Taipei, Taiwan

152 Department of Physics, Technion: Israel Institute of Technology, Haifa, Israel

153 Raymond and Beverly Sackler School of Physics and Astronomy, Tel Aviv University, Tel Aviv, Israel

154 Department of Physics, Aristotle University of Thessaloniki, Thessaloniki, Greece

155 International Center for Elementary Particle Physics and Department of Physics, The University of Tokyo, Tokyo, Japan

156 Graduate School of Science and Technology, Tokyo Metropolitan University, Tokyo, Japan

157 Department of Physics, Tokyo Institute of Technology, Tokyo, Japan

158 Department of Physics, University of Toronto, Toronto ON, Canada

159 (a) TRIUMF, Vancouver BC; (b) Department of Physics and Astronomy, York University, Toronto ON, Canada

160 Faculty of Pure and Applied Sciences, University of Tsukuba, Tsukuba, Japan

161 Department of Physics and Astronomy, Tufts University, Medford MA, United States of America

162 Centro de Investigaciones, Universidad Antonio Narino, Bogota, Colombia

163 Department of Physics and Astronomy, University of California Irvine, Irvine CA, United States of America

164 (a) INFN Gruppo Collegato di Udine; ${ }^{(b)}$ ICTP, Trieste; ${ }^{(c)}$ Dipartimento di Chimica, Fisica e Ambiente, Università di Udine, Udine, Italy

165 Department of Physics, University of Illinois, Urbana IL, United States of America

166 Department of Physics and Astronomy, University of Uppsala, Uppsala, Sweden

167 Instituto de Física Corpuscular (IFIC) and Departamento de Física Atómica, Molecular y Nuclear and Departamento de Ingeniería Electrónica and Instituto de Microelectrónica de Barcelona (IMB-CNM), University of Valencia and CSIC, Valencia, Spain

168 Department of Physics, University of British Columbia, Vancouver BC, Canada

169 Department of Physics and Astronomy, University of Victoria, Victoria BC, Canada

170 Department of Physics, University of Warwick, Coventry, United Kingdom

171 Waseda University, Tokyo, Japan

172 Department of Particle Physics, The Weizmann Institute of Science, Rehovot, Israel

173 Department of Physics, University of Wisconsin, Madison WI, United States of America

174 Fakultät für Physik und Astronomie, Julius-Maximilians-Universität, Würzburg, Germany

175 Fachbereich C Physik, Bergische Universität Wuppertal, Wuppertal, Germany

176 Department of Physics, Yale University, New Haven CT, United States of America

177 Yerevan Physics Institute, Yerevan, Armenia

178 Centre de Calcul de l'Institut National de Physique Nucléaire et de Physique des Particules (IN2P3), Villeurbanne, France

a Also at Department of Physics, King's College London, London, United Kingdom

b Also at Laboratorio de Instrumentacao e Fisica Experimental de Particulas - LIP, Lisboa, Portugal

c Also at Faculdade de Ciencias and CFNUL, Universidade de Lisboa, Lisboa, Portugal

d Also at Particle Physics Department, Rutherford Appleton Laboratory, Didcot, United Kingdom

e Also at Department of Physics, University of Johannesburg, Johannesburg, South Africa

$f$ Also at TRIUMF, Vancouver BC, Canada

$g$ Also at Department of Physics, California State University, Fresno CA, United States of America

h Also at Novosibirsk State University, Novosibirsk, Russia

${ }^{i}$ Also at Department of Physics, University of Coimbra, Coimbra, Portugal

$j$ Also at Department of Physics, UASLP, San Luis Potosi, Mexico

$k$ Also at Università di Napoli Parthenope, Napoli, Italy

$l$ Also at Institute of Particle Physics (IPP), Canada

$m$ Also at Department of Physics, Middle East Technical University, Ankara, Turkey

$n$ Also at Louisiana Tech University, Ruston LA, United States of America 
o Also at Dep Fisica and CEFITEC of Faculdade de Ciencias e Tecnologia, Universidade Nova de Lisboa, Caparica, Portugal

$p$ Also at Department of Physics and Astronomy, University College London, London, United Kingdom

$q$ Also at Department of Physics, University of Cape Town, Cape Town, South Africa

${ }^{r}$ Also at Institute of Physics, Azerbaijan Academy of Sciences, Baku, Azerbaijan

$s$ Also at Institut für Experimentalphysik, Universität Hamburg, Hamburg, Germany

${ }^{t}$ Also at Manhattan College, New York NY, United States of America

$u$ Also at CPPM, Aix-Marseille Université and CNRS/IN2P3, Marseille, France

$v$ Also at School of Physics and Engineering, Sun Yat-sen University, Guanzhou, China

w Also at Academia Sinica Grid Computing, Institute of Physics, Academia Sinica, Taipei, Taiwan

$x$ Also at School of Physics, Shandong University, Shandong, China

y Also at Dipartimento di Fisica, Università La Sapienza, Roma, Italy

$z$ Also at DSM/IRFU (Institut de Recherches sur les Lois Fondamentales de l'Univers), CEA Saclay (Commissariat à l'Energie Atomique et aux Energies Alternatives), Gif-sur-Yvette, France

a Also at section de Physique, Université de Genève, Geneva, Switzerland

ab Also at Departamento de Fisica, Universidade de Minho, Braga, Portugal

ac Also at Department of Physics and Astronomy, University of South Carolina, Columbia SC, United States of America

ad Also at Institute for Particle and Nuclear Physics, Wigner Research Centre for Physics, Budapest, Hungary

ae Also at California Institute of Technology, Pasadena CA, United States of America

af Also at Institute of Physics, Jagiellonian University, Krakow, Poland

ag Also at LAL, Université Paris-Sud and CNRS/IN2P3, Orsay, France

ah Also at Nevis Laboratory, Columbia University, Irvington NY, United States of America

ai Also at Department of Physics and Astronomy, University of Sheffield, Sheffield, United Kingdom

aj Also at Department of Physics, Oxford University, Oxford, United Kingdom

ak Also at Department of Physics, The University of Michigan, Ann Arbor MI, United States of America

al Also at Discipline of Physics, University of KwaZulu-Natal, Durban, South Africa

am Also at Institute of Physics, Academia Sinica, Taipei, Taiwan

* Deceased 